\title{
Cytotoxic Guanidine Alkaloids from a French Polynesian Monanchora n. sp. Sponge
}

Amr El-Demerdash,,$^{\ddagger \ddagger}$ Céline Moriou, ${ }^{\dagger}$ Marie-Thérèse Martin, ${ }^{\dagger}$ Alice de Souza RodriguesStien, ${ }^{\dagger}$ Sylvain Petek,${ }^{\S}$ Marina Demoy-Schneider,${ }^{\perp}$ Kathryn Hall, ${ }^{\prime}$ John N. A. Hooper, ${ }^{\prime \prime}{ }^{\circ}$ Cécile Debitus $^{\S}$ and Ali Al-Mourabit ${ }^{\dagger *}$

\footnotetext{
${ }^{\dagger}$ Institut de Chimie des Substances Naturelles, CNRS UPR 2301, Univ. Paris-Sud, Université Paris-Saclay, 1, av. de la Terrasse, 91198 Gif-sur-Yvette (France).

${ }^{\ddagger}$ Organic Chemistry Division, Chemistry Department, Faculty of Science, Mansoura University, Mansoura35516-Egypt.

${ }^{\S}$ Institut de Recherche pour le Développement (IRD), UMR-241 EIO, BP529, 98713, Papeete, Tahiti, French Polynesia.

${ }^{\perp}$ Université de la Polynésie française, UMR-241 EIO, BP 6570, 98702 Faa’a Aéroport, Tahiti, French Polynesia

"Queensland Museum, PO box 3300, South Brisbane BC, Queensland 4101, Australia

- Eskitis Institute for Drug Discovery, Griffith University, Nathan, Queensland 4111, Australia
}

- Corresponding Author, Tel: 31698245 85: FAX: 3316907 72 47: E-Mail: ali.almourabit@cnrs.fr 
Page 4: Monanchora sp. (OTU QM4696) identification and taxonomic considerations

Page 6: Monanchora sp. in situ picture and structures of the isolated molecules

Page 7-9: observed data for the known compounds 12-15

\section{Page 10-71: NMR and CD spectra}

S0. Observed data for known compounds.

S1. ${ }^{1} \mathrm{H}$ NMR spectrum of monanchoradin $\mathrm{A}(\mathbf{1})$ in $\mathrm{CD}_{3} \mathrm{OD}(500 \mathrm{MHz})$.

S2. ${ }^{13} \mathrm{C}$ NMR spectrum of monanchoradin $\mathrm{A}(\mathbf{1})$ in $\mathrm{CD}_{3} \mathrm{OD}(125 \mathrm{MHz})$.

S3. ${ }^{1} \mathrm{H}-{ }^{1} \mathrm{H}$ COSY NMR spectrum of monanchoradin A (1) in $\mathrm{CD}_{3} \mathrm{OD}(500 \mathrm{MHz})$.

S4. HSQC NMR spectrum of monanchoradin A (1) in $\mathrm{CD}_{3} \mathrm{OD}(500 \mathrm{MHz})$.

S5. ${ }^{1} \mathrm{H}-{ }^{13} \mathrm{C}$ HMBC NMR spectrum of monanchoradin $\mathrm{A}(\mathbf{1})$ in $\mathrm{CD}_{3} \mathrm{OD}(500 \mathrm{MHz})$.

S6. UV spectrum of monanchoradin A (1) in $\mathrm{MeOH}$.

S7. IR spectrum of monanchoradin A (1).

S8. ${ }^{1} \mathrm{H}$ NMR spectrum of monanchorodin B (2) in (500 MHz).

S9. ${ }^{1} \mathrm{H}$ NMR spectrum of monanchorodin C (3) in DMF-D 6 (600 MHz).

S10. ${ }^{13} \mathrm{C}$ NMR spectrum of monanchorodin C (3) in in DMF $(150 \mathrm{MHz})$.

S11. ${ }^{1} \mathrm{H}-{ }^{1} \mathrm{H}$ COSY NMR spectrum of monanchorodin C (3) in DMF-D 6 (600 MHz).

S12. HSQC NMR spectrum of monanchorodin C (3) in DMF-D 6 (600 MHz).

S13. ${ }^{1} \mathrm{H}_{-}{ }^{13} \mathrm{C}$ HMBC NMR spectrum of monanchorodin C (3) in $\mathrm{DMF}_{-} \mathrm{D}_{6}(600 \mathrm{MHz})$.

S14. UV spectrum of monanchorodin $\mathrm{C}(3)$ in $\mathrm{MeOH}$.

S15. IR spectrum of monanchorodin C (3).

S16. ${ }^{1} \mathrm{H}$ NMR comparison for monanchoradin A (1), monanchoradin B (2) and monanchoradin $\mathrm{C}(\mathbf{3})$ in $\mathrm{CD}_{3} \mathrm{OD}(500 \mathrm{MHz})$.

S17. ${ }^{1} \mathrm{H}$ NMR spectrum of dehydrocrambescin $\mathrm{A} 2418$ (4) in $\mathrm{CD}_{3} \mathrm{OH}(500 \mathrm{MHz})$.

S18. ${ }^{13} \mathrm{C}$ NMR spectrum of dehydrocrambescin $\mathrm{A} 2418$ (4) in $\mathrm{CD}_{3} \mathrm{OH}(125 \mathrm{MHz})$.

S19. ${ }^{1} \mathrm{H}-{ }^{1} \mathrm{H}$ COSY NMR spectrum of dehydrocrambescin A2 418 (4) in $\mathrm{CD}_{3} \mathrm{OH}(500 \mathrm{MHz})$.

S20. HSQC NMR spectrum of dehydrocrambescin A2 418 (4) in $\mathrm{CD}_{3} \mathrm{OH}(500 \mathrm{MHz})$.

S21. ${ }^{1} \mathrm{H}_{-}{ }^{13} \mathrm{C}$ HMBC NMR spectrum of dehydrocrambescin $\mathrm{A} 2418$ (4) in $\mathrm{CD}_{3} \mathrm{OH}(500 \mathrm{MHz})$. 
S22. UV spectrum of dehydrocrambescin A2 418 (4) in $\mathrm{MeOH}$.

S23. IR spectrum of dehydrocrambescin A2 418 (4).

S24. Variation of ${ }^{1} \mathrm{H}$ NMR of dehydrocrambescin $\mathrm{A} 2418$ (4) with time in $\mathrm{CD}_{3} \mathrm{OD}$ (500 $\mathrm{MHz})$.

S25. Hydrogen deuterium exchange mechanism.

S26. ${ }^{1} \mathrm{H}$ NMR spectrum of crambescidin $786(5)$ in $\mathrm{CD}_{3} \mathrm{OD}(500 \mathrm{MHz})$.

S27. ${ }^{13} \mathrm{C}$ NMR spectrum of crambescidin $786(5)$ in $\mathrm{CD}_{3} \mathrm{OD}(125 \mathrm{MHz})$.

S28. ${ }^{1} \mathrm{H}-{ }^{1} \mathrm{H}$ COSY NMR spectrum of crambescidin $786(5)$ in $\mathrm{CD}_{3} \mathrm{OD}(500 \mathrm{MHz})$.

S29. HSQC NMR spectrum of crambescidin $786(5)$ in $\mathrm{CD}_{3} \mathrm{OD}(500 \mathrm{MHz})$.

S30. UV spectrum of crambescidin 786 (5) in $\mathrm{MeOH}$.

S31. IR spectrum of crambescidin 786 (5).

S32. ${ }^{1} \mathrm{H}$ NMR spectrum of crambescidin 814 (6) in $\mathrm{CD}_{3} \mathrm{OD}(500 \mathrm{MHz})$.

S33. ${ }^{13} \mathrm{C}$ NMR spectrum of crambescidin 814 (6) in $\mathrm{CD}_{3} \mathrm{OD}(125 \mathrm{MHz})$.

S34. ${ }^{1} \mathrm{H}-{ }^{1} \mathrm{H}$ COSY NMR spectrum of crambescidin 814 (6) in $\mathrm{CD}_{3} \mathrm{OD}(500 \mathrm{MHz})$.

S35. HSQC NMR spectrum of crambescidin 814 (6) in $\mathrm{CD}_{3} \mathrm{OD}(500 \mathrm{MHz})$.

S36. ${ }^{1} \mathrm{H}-{ }^{13} \mathrm{C}$ HMBC NMR spectrum of crambescidin 814 (6) in $\mathrm{CD}_{3} \mathrm{OD}(500 \mathrm{MHz})$.

S37. UV spectrum of crambescidin 814 (6) in $\mathrm{MeOH}$.

S38. IR spectrum of crambescidin 814 (6).

S39. ${ }^{1} \mathrm{H}$ NMR comparison of crambescidin $786(5)$, crambescidin 800 (12) and crambescidin 814 (7) in $\mathrm{CD}_{3} \mathrm{OD}(500 \mathrm{MHz})$.

S40. ${ }^{1} \mathrm{H}$ NMR spectrum of norcrambescidic acid (7) in $\mathrm{CD}_{3} \mathrm{OD}(600 \mathrm{MHz})$.

S41. ${ }^{13} \mathrm{C}$ NMR spectrum of norcrambescidic acid (7) in $\mathrm{CD}_{3} \mathrm{OD}(150 \mathrm{MHz})$.

S42. ${ }^{1} \mathrm{H}-{ }^{1} \mathrm{H}$ COSY NMR spectrum of norcrambescidic acid (7) in $\mathrm{CD}_{3} \mathrm{OD}(600 \mathrm{MHz})$.

S43. HSQC NMR spectrum of norcrambescidic acid (7) in $\mathrm{CD}_{3} \mathrm{OD}(600 \mathrm{MHz})$.

S44. ${ }^{1} \mathrm{H}-{ }^{13} \mathrm{C}$ HMBC NMR spectrum of norcrambescidic acid (7) in $\mathrm{CD}_{3} \mathrm{OD}(600 \mathrm{MHz})$.

S45. UV spectrum of norcrambescidic acid (7) in $\mathrm{MeOH}$.

S46. IR spectrum of norcrambescidic acid (7).

S47. ${ }^{1} \mathrm{H}$ NMR spectrum of monalidine A (7) in CD3OD (500 MHz). 
S48. ${ }^{13} \mathrm{C}$ NMR spectrum of monalidine A (7) in CD3OD (125 MHz).

S49. ${ }^{1} \mathrm{H}$ NMR spectrum of (-)-crambescin A2 392 (9) in $\mathrm{CD}_{3} \mathrm{OD}(500 \mathrm{MHz})$.

S50. ${ }^{13} \mathrm{C}$ NMR spectrum of (-)-crambescin $\mathrm{A} 2392(9)$ in $\mathrm{CD}_{3} \mathrm{OD}(125 \mathrm{MHz})$.

S51. ${ }^{1} \mathrm{H}$ NMR spectrum of (-)-crambescin $\mathrm{A} 2406$ (10) in $\mathrm{CD}_{3} \mathrm{OD}(500 \mathrm{MHz})$.

S52. ${ }^{13} \mathrm{C}$ NMR spectrum of (-)-crambescin $\mathrm{A} 2406$ (10) in $\mathrm{CD}_{3} \mathrm{OD}(125 \mathrm{MHz})$.

S53. ${ }^{1} \mathrm{H}$ NMR spectrum of (-)-crambescin A2 420 (11)in $\mathrm{CD}_{3} \mathrm{OD}(500 \mathrm{MHz})$.

S54. ${ }^{13} \mathrm{C}$ NMR spectrum of (-)-crambescin $\mathrm{A} 2420$ (11) in $\mathrm{CD}_{3} \mathrm{OD}(125 \mathrm{MHz})$.

S55. ${ }^{1} \mathrm{H}$ NMR comparison for (-)-crambescins A2 392 (9), 406 (10) and 420 (11) in $\mathrm{CD}_{3} \mathrm{OD}$ $(500 \mathrm{MHz})$.

S56. ECD spectra for (-)-crambescins A2 392 (9), 406 (10) and 420 (11) as TFA salt.

S57. ${ }^{1} \mathrm{H}$ NMR spectrum of crambescidin 800 (12) in $\mathrm{CD}_{3} \mathrm{OD}(500 \mathrm{MHz})$.

S58. ${ }^{13} \mathrm{C}$ NMR spectrum of crambescidin $800(\mathbf{1 2})$ in $\mathrm{CD}_{3} \mathrm{OD}(125 \mathrm{MHz})$.

S59. ${ }^{1} \mathrm{H}$ NMR spectrum of crambescidin $826(\mathbf{1 3})$ in $\mathrm{CD}_{3} \mathrm{OD}(500 \mathrm{MHz})$.

S60. ${ }^{13} \mathrm{C}$ NMR spectrum of crambescidin $826(\mathbf{1 3})$ in $\mathrm{CD}_{3} \mathrm{OD}(125 \mathrm{MHz})$.

S61. ${ }^{1} \mathrm{H}$ NMR spectrum of crambescidic acid (14) in $\mathrm{CD}_{3} \mathrm{OD}(500 \mathrm{MHz})$.

S62. ${ }^{13} \mathrm{C}$ NMR spectrum of crambescidic acid (14) in $\mathrm{CD}_{3} \mathrm{OD}(125 \mathrm{MHz})$.

S63. ${ }^{1} \mathrm{H}$ NMR comparison of (-)-20-norcrambescidic acid (7) and crambescidic acid (14) in $\mathrm{CD}_{3} \mathrm{OD}(500 \mathrm{MHz})$.

S64. ${ }^{1} \mathrm{H}$ NMR spectrum of crambescidin 359 (15) in $\mathrm{CD}_{3} \mathrm{OD}(500 \mathrm{MHz})$.

S65. ${ }^{13} \mathrm{C}$ NMR spectrum of crambescidin $359(\mathbf{1 5})$ in $\mathrm{CD}_{3} \mathrm{OD}(125 \mathrm{MHz})$.

S66. ECD spectra for (-)-crambescidins $(\mathbf{6 , 1 2}, \mathbf{1 4}, \mathbf{1 5})$, and the (-)-20-norcrambescidin (7).

S67. ${ }^{1} \mathrm{H}$ NMR comparison of crambescidin 800 (12) and crambescidin 814 (6) in $\mathrm{CD}_{3} \mathrm{OD}(500$ $\mathrm{MHz})$. 
Class Demospongiae Sollas, 1885

Subclass Heteroscleromorpha Cárdenas, Perez \& Boury-Esnault, 2012

Order Poecilosclerida Topsent, 1928

Family Crambeidae Lévi, 1963

\section{Monanchora sp. (OTU QM4696)}

\section{Description}

Growth form: thickly encrusting, anastomosing over substrate, lumpy bulbous surface when alive.

Colour: red in life; beige, with tinges of orange at the apices of bulbs when preserved.

Oscules: moderately large, typically $5 \mathrm{~mm}$ diameter when alive but up to $10 \mathrm{~mm}$ diameter in some parts of the surface; each oscule is surrounded by a prominent membranous lip pigmented the same red colour as the rest of the sponge; oscules are predominantly located along the apex of ridges and bulbous surface lobes; contracted in ethanol.

Texture: compressible, dense, not resilient.

Surface: smooth; slightly undulating; ectosome clearly differentiated, but cannot be peeled away; choanosome dense, pliable.

Ectosomal skeleton: thick, dense cortex of megascleres in paratangential, thick wavy tracts. Tracts surrounding the oscules are perpendicular to the surface, supporting the soft oscular lip. Chelae appear to be restricted to the ectosomal region, particularly immediately subcutaneous, associated with oscules in particular, and near the tips of the projecting tracts of megascleres.

Choanosomal skeleton: confused, spicules less dense than in the ectosomal skeleton, reinforced by large fibres; mesohyl light, consistent.

Megascleres: subtylostyles with slightly swollen tylote bases and abruptly rounded points, without much obvious taper, shafts slender, predominantly straight but occasionally curved in younger (thinner) spicules; range 185-284 micrometres long, 2-5 micrometres shaft diameter.

Microscleres: unquiferous anchorate isochelae: reduced with 4 or 5 small alae (teeth), shafts very straight, range 20-28 micrometres long. (from Hall et al. 2015)

Remarks. There are 11 described, valid species of Monanchora known worldwide (Van Soest et al. 2015), of which the present species most closely resembles Monanchora unguiculata (Dendy, 1922) (type locality Egmont Atoll, Chagos Archipelago, Indian Ocean), with other records from the Indian Ocean, the IndoMalay Archipelago and the central north Pacific as far east as Pohnpei, Caroline Islands, Federated States of Micronesia. The Pohnpei population (described as 
Folispa pingens de Laubenfels, 1954, but synonymised into Monanchora unguiculata by Van Soest 1990) has two categories of strongly curved anchorate chelae, the larger category well-silicified and 30 micrometres chord length, and the smaller poorly silicified and aroun 15 micrometres chord length. The present species from the remote Tahuata, Marquesas Islands, French Polynesia, differs from that (and all other described species of Monanchora), by its possession of a single category of very straight, unquiferous anchorate isochelae that have rudimentary alae but well developed shafts, 20-28 micrometers long. The present species probably represents a new species but requires molecular barcoding to verify whether these morphological differences from $M$. unquiculata are significant or otherwise. This is the first record of a Monanchora species from the Central South Pacific Ocean.

\section{Material:}

Registration Number: Queensland Museum, Brisbane, G331116

Locality: Tahuata, Marquesas Islands

Nation: French Polynesia

Latitude: -9.86694

Longitude: -139.020972

Depth (from) (m): 20 metres

Collection date: $11 / 09 / 2009$

Habitat: vertical cliff

Collection method: SCUBA

\section{Sources}

De Laubenfels, M.W. (1954) The sponges of the West-Central Pacific. Oregon State Monographic Studies in Zoology 7:1-320

Hall, K.A., Ekins, M. \& Hooper, J.N.A. (2015). QM4696 Monanchora sp. (OTU QM4696). In: Hall, K.A. \& Hooper, J.N.A. (2015) SpongeMaps: an online community for sponge taxonomy. Internet resource, available at http://www.spongemaps.org [last accessed: 4/12/2015]

Van Soest, R.W.M. 1990a. Monanchora stocki n. sp. (Porifera, Poecilosclerida) from the Mid-Atlantic Islands. Bijdragen tot de Dierkunde 60 (3-4): 249-255

Van Soest, R.W.M; Boury-Esnault, N.; Hooper, J.N.A.; Rützler, K.; de Voogd, N.J.; Alvarez de Glasby, B.; Hajdu, E.; Pisera, A.B.; Manconi, R.; Schoenberg, C.; Janussen, D.; Tabachnick, K.R., Klautau, M.; Picton, B.; Kelly, M.; Vacelet, J.; Dohrmann, M.; Díaz, M.-C.; Cárdenas, P. (2015) World Porifera database. Accessed at http://www.marinespecies.org/porifera on 2015-12-04 


\section{In-situ Monanchora $\mathrm{n}$. sp photo}

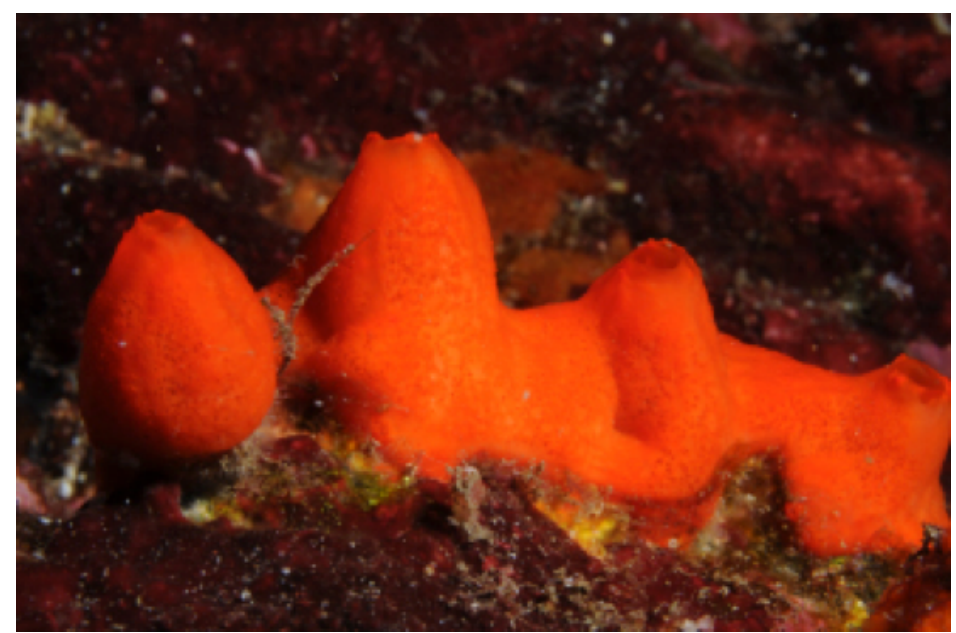

\section{Isolation Scheme}

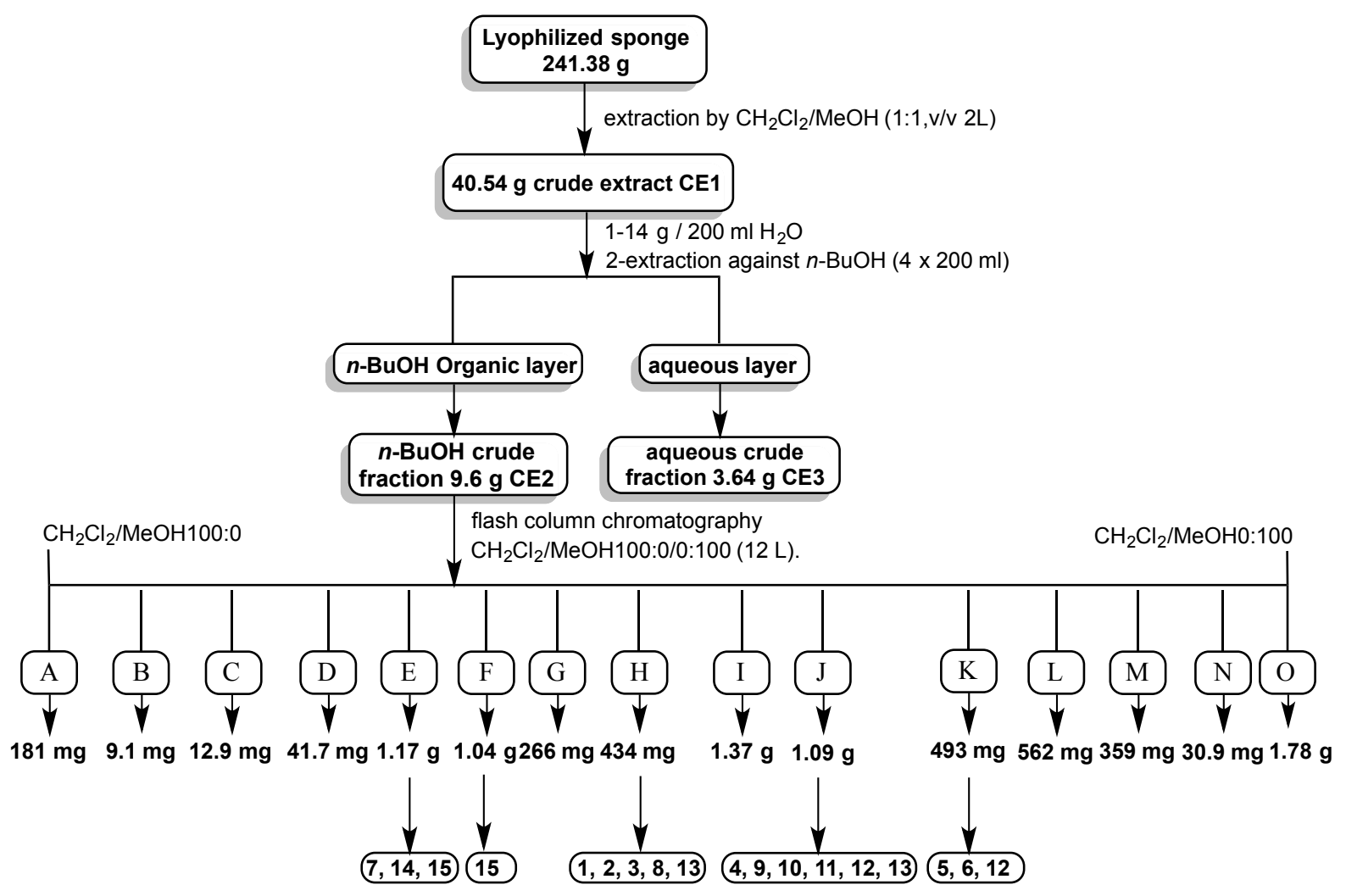

Isolated quantities: $\mathbf{1}(4.5 \mathrm{mg}), \mathbf{2}(0.5 \mathrm{mg}), \mathbf{3}(0.7 \mathrm{mg}), \mathbf{4}(7.0 \mathrm{mg}), \mathbf{5}(5.0 \mathrm{mg}), \mathbf{6}(6.6 \mathrm{mg}, \mathbf{7}$

(1.0 mg), 8 (9.2 mg), $9(10.8 \mathrm{mg}), \mathbf{1 0}(5.5 \mathrm{mg}), \mathbf{1 1}(32.2 \mathrm{mg}), \mathbf{1 2}(277.2 \mathrm{mg}), \mathbf{1 3}(80.9 \mathrm{mg}), \mathbf{1 4}$

(10.3 mg), 15 (9.4 mg) 
Compounds (1-15) isolated from the French Polynesian Sponge Monanchora $\mathrm{n} . \mathrm{sp}$

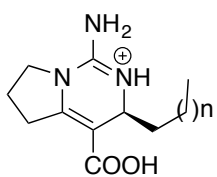

monanchoradin $A(1), n=5$ monanchoradin $\mathrm{B}(2), \mathrm{n}=6$ monanchoradin $C(3), n=7$

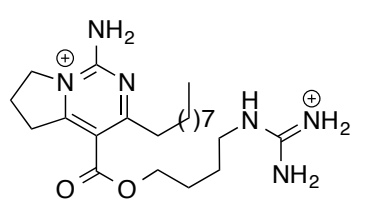

dehydrocrambescin A2 418 (4)

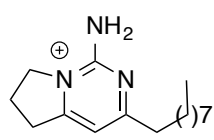

monalidine $A(8)$

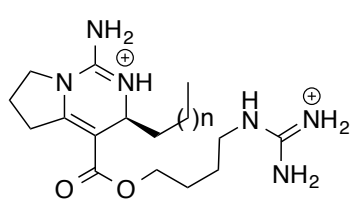

(-)-crambescin A2 $392(9), \mathrm{n}=5$ (-)-crambescin A2 406 (10), $n=6$ (-)-crambescin A2 420 (11), $n=7$

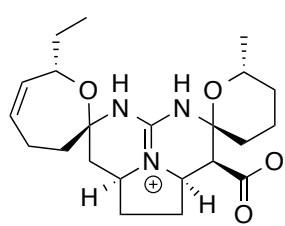

crambescidin $786(5), n=12$ crambescidin $814(6), \mathrm{n}=14$ crambescidin $800(\mathbf{1 2}), \mathrm{n}=13$

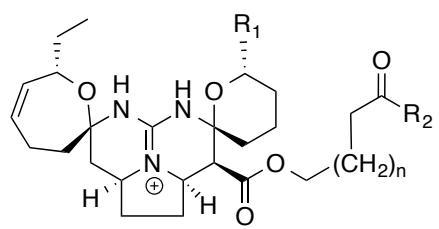

$\mathrm{H}^{\prime}$

$\mathrm{H}$ O
$\mathrm{OH}$

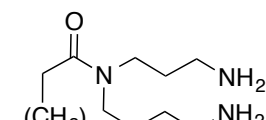

$\mathrm{OH}$

crambescidin 826 (13), $n=15, \mathrm{R}_{1}=\mathrm{CH}_{3}, \mathrm{R}_{2}=$ - $\mathrm{N}$ crambescidic acid (14), $n=13, R_{1}=C_{3}, R_{2}=O H$ norcrambescidic acid (7), $n=13, \mathrm{R}_{1}=\mathrm{H}, \mathrm{R}_{2}=\mathrm{OH}$

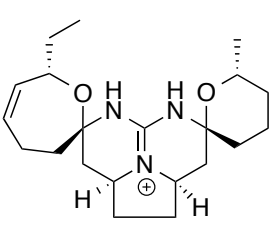

crambescidin 359 (15) 
S0. Observed data for known compounds.

(-)-Crambescidin 800 (12): pale yellow oil (11 mg); $[\alpha]^{25} \mathrm{D}-7(c 0.70, \mathrm{MeOH}$ as formate salt), $[\alpha]^{25} \mathrm{D}-3.5\left(c \quad 0.72, \mathrm{CHCl}_{3}\right.$ as $\mathrm{HCl}$ salt); UV (MeOH) $\lambda_{\max }(\log \varepsilon) 218(1.60) \mathrm{nm} ; \mathrm{ECD}(0.02 \mathrm{mg} / \mathrm{mL}$, $\mathrm{MeOH}), \lambda_{\max }(\Delta \varepsilon) 204(15.03) \mathrm{nm}$; IR (neat) $v_{\max }$ 2922, 2852, 2674, 1731, 1628, 1341, 1161, 1016, 753, 718, $662 \mathrm{~cm}^{-1} ;{ }^{1 H}$ NMR and ${ }^{13} \mathrm{C}$ NMR data see Supporting Information; HRESIMS $\mathrm{m} / \mathrm{z}$ $801.6205[\mathrm{M}+\mathrm{H}]^{+}\left(\right.$calcd for $\left.\mathrm{C}_{45} \mathrm{H}_{81} \mathrm{~N}_{6} \mathrm{O}_{6}, 801.6218\right)$.

(-)-Crambescidin 826 (13): yellow oil (80.9 mg); [ $\alpha]^{25} \mathrm{D}-11(c 0.50, \mathrm{MeOH}$, as formate salt); UV $(\mathrm{MeOH}) \lambda_{\max }(\log \varepsilon) 215(1.10) \mathrm{nm} ; \mathrm{ECD}(0.02 \mathrm{mg} / \mathrm{mL}, \mathrm{MeOH}), \lambda_{\max }(\Delta \varepsilon) 203$ (12.74) nm; IR (neat) $v_{\max } 2923,2852,2775,1731,1626,1580,1459,1370,1340,1162,1040,1016,755,717,660 \mathrm{~cm}^{-1} ;{ }^{1} \mathrm{H}$ NMR and ${ }^{13} \mathrm{C}$ NMR data see Supporting Information; HRESIMS $m / z$ z27.6473 [M + H]+ (calcd for $\mathrm{C}_{47} \mathrm{H}_{83} \mathrm{~N}_{6} \mathrm{O}_{6}, 827.6374$ ).

(-)-Crambescidic acid (14): pale yellow powder (10.3 mg); $[\alpha]{ }^{25} \mathrm{D}-3(c 0.44, \mathrm{MeOH}$, as formate salt); UV (MeOH) $\lambda_{\max }(\log \varepsilon) 201.0(0.85) \mathrm{nm} ; \mathrm{ECD}(0.02 \mathrm{mg} / \mathrm{mL}, \mathrm{MeOH}), \lambda_{\max }(\Delta \varepsilon) 204(12.20)$ nm; IR (neat) $v_{\max } 3232$ (broad band, COOH), 3126, 2924, 2853, 1731, 1657, 1609, 1167, 1015, 970, $716 \mathrm{~cm}^{-1} ;{ }^{1} \mathrm{H}$ NMR and ${ }^{13} \mathrm{C}$ NMR data see Supporting Information); HRESIMS $\mathrm{m} / \mathrm{z}$ 658.4641 [M $+\mathrm{H}]^{+}\left(\right.$calcd for $\left.\mathrm{C}_{38} \mathrm{H}_{64} \mathrm{~N}_{3} \mathrm{O}_{6}, 658.4795\right)$.

(-)-Crambescidin 359 (15): yellow oil (6.3 mg); $[\alpha]^{25} \mathrm{D}-6(c \quad 0.36, \mathrm{MeOH}$, as formate salt); UV $(\mathrm{MeOH}) \lambda_{\max }(\log \varepsilon) 210.5(1.00) \mathrm{nm} ; \mathrm{ECD}(0.02 \mathrm{mg} / \mathrm{mL}, \mathrm{MeOH}), \lambda_{\max }(\Delta \varepsilon) 203$ (7.09) nm; IR (neat) $v_{\max } 2967,2932,1630,1542,1448,1343,1114,1054,1010,965,844,754 \mathrm{~cm}^{-1} ;{ }^{1} \mathrm{H}$ NMR and ${ }^{13} \mathrm{C}$ NMR data see Supporting Information); HRESIMS m/z $360.2529[\mathrm{M}+\mathrm{H}]^{+}$(calcd for $\left.\mathrm{C}_{21} \mathrm{H}_{34} \mathrm{~N}_{3} \mathrm{O}_{2}, 360.2651\right)$. 
S1. ${ }^{1} \mathrm{H}$ NMR spectrum of monanchoradin $\mathrm{A}(1)$ in $\mathrm{CD}_{3} \mathrm{OD}(500 \mathrm{MHz})$.

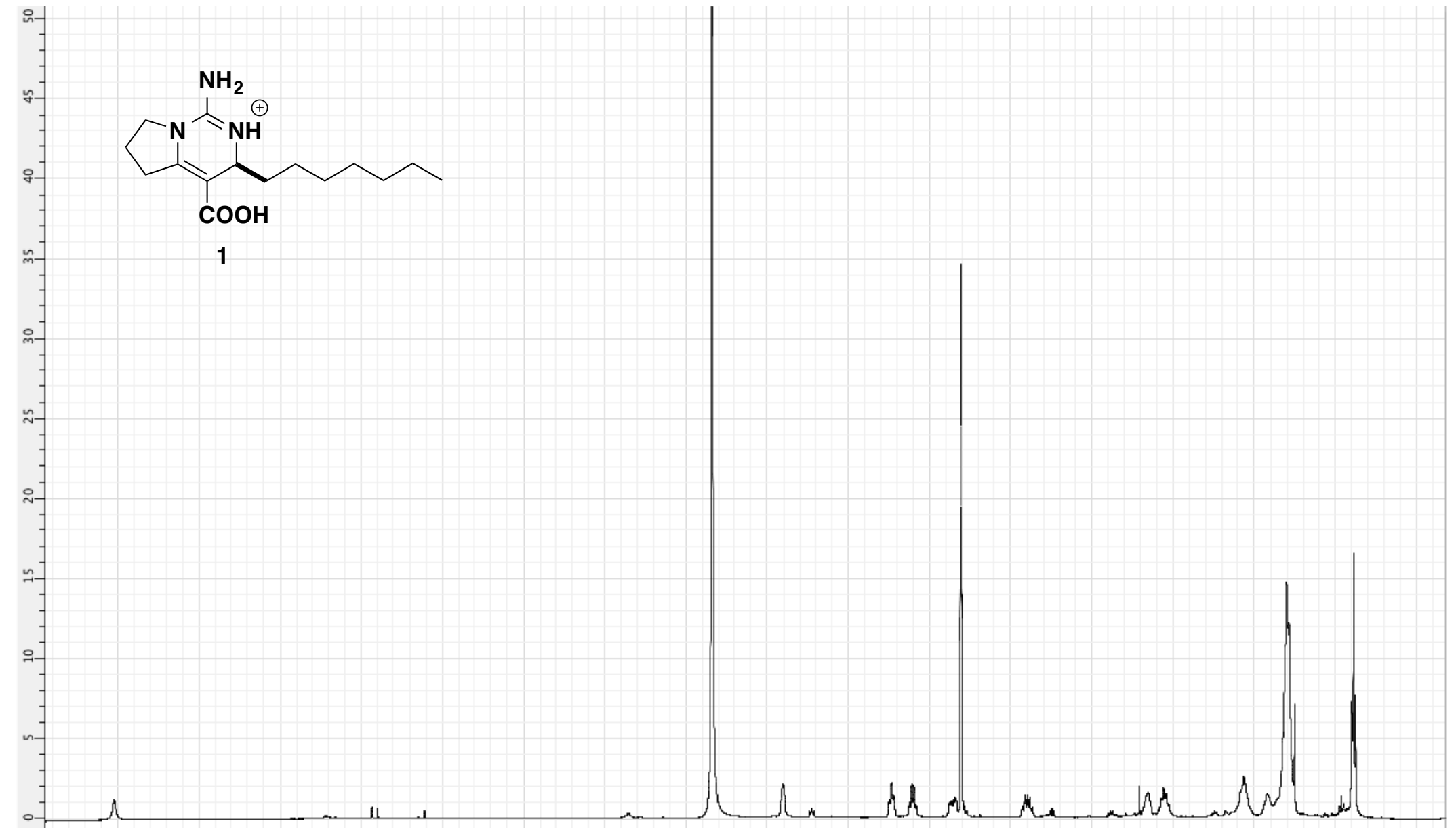


S2. ${ }^{13} \mathrm{C}$ NMR spectrum of monanchoradin $\mathrm{A}(\mathbf{1})$ in $\mathrm{CD}_{3} \mathrm{OD}(125 \mathrm{MHz})$.

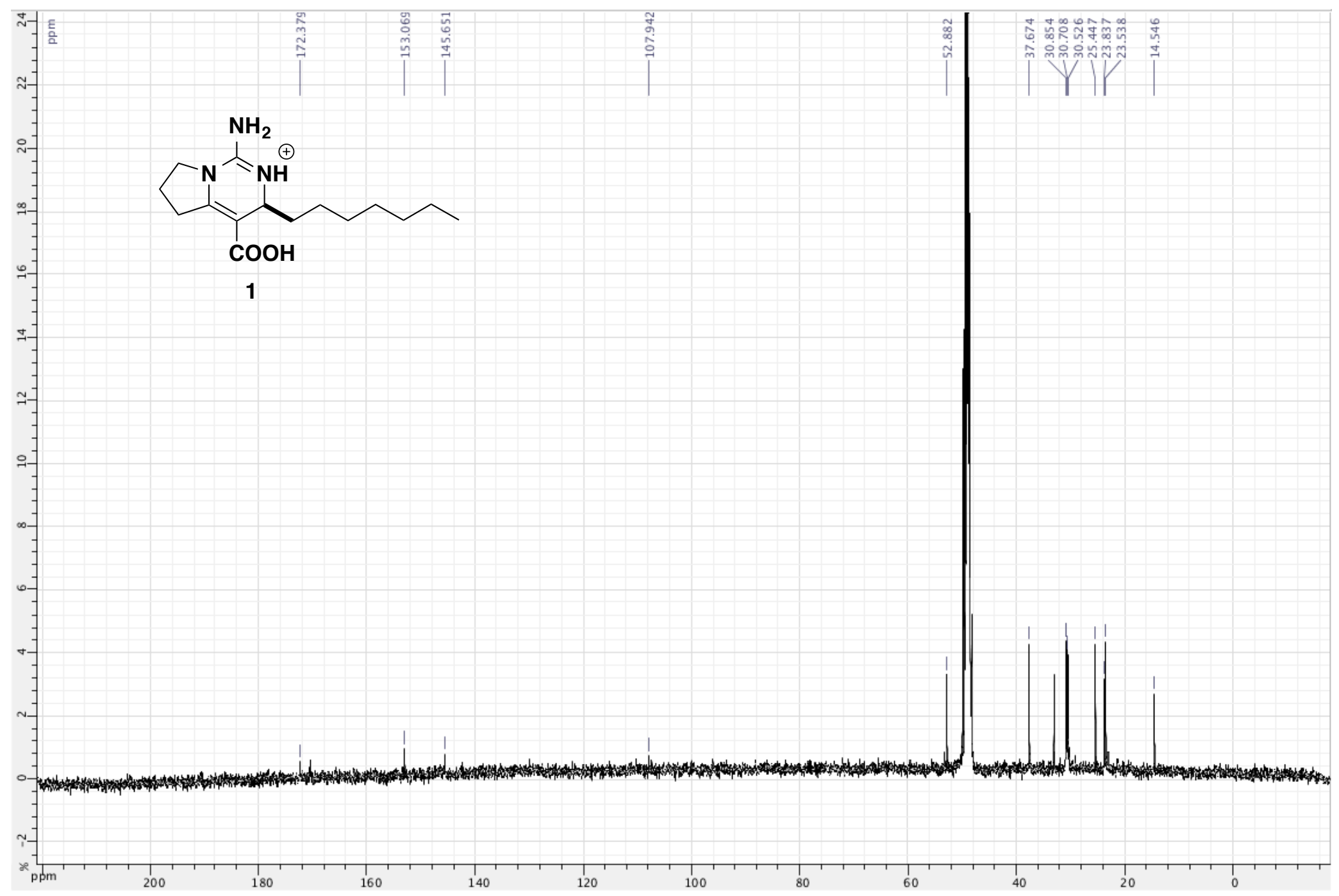


S3. ${ }^{1} \mathrm{H}-{ }^{1} \mathrm{H}$ COSY NMR spectrum of monanchoradin $\mathrm{A}(\mathbf{1})$ in $\mathrm{CD}_{3} \mathrm{OD}(500 \mathrm{MHz})$.

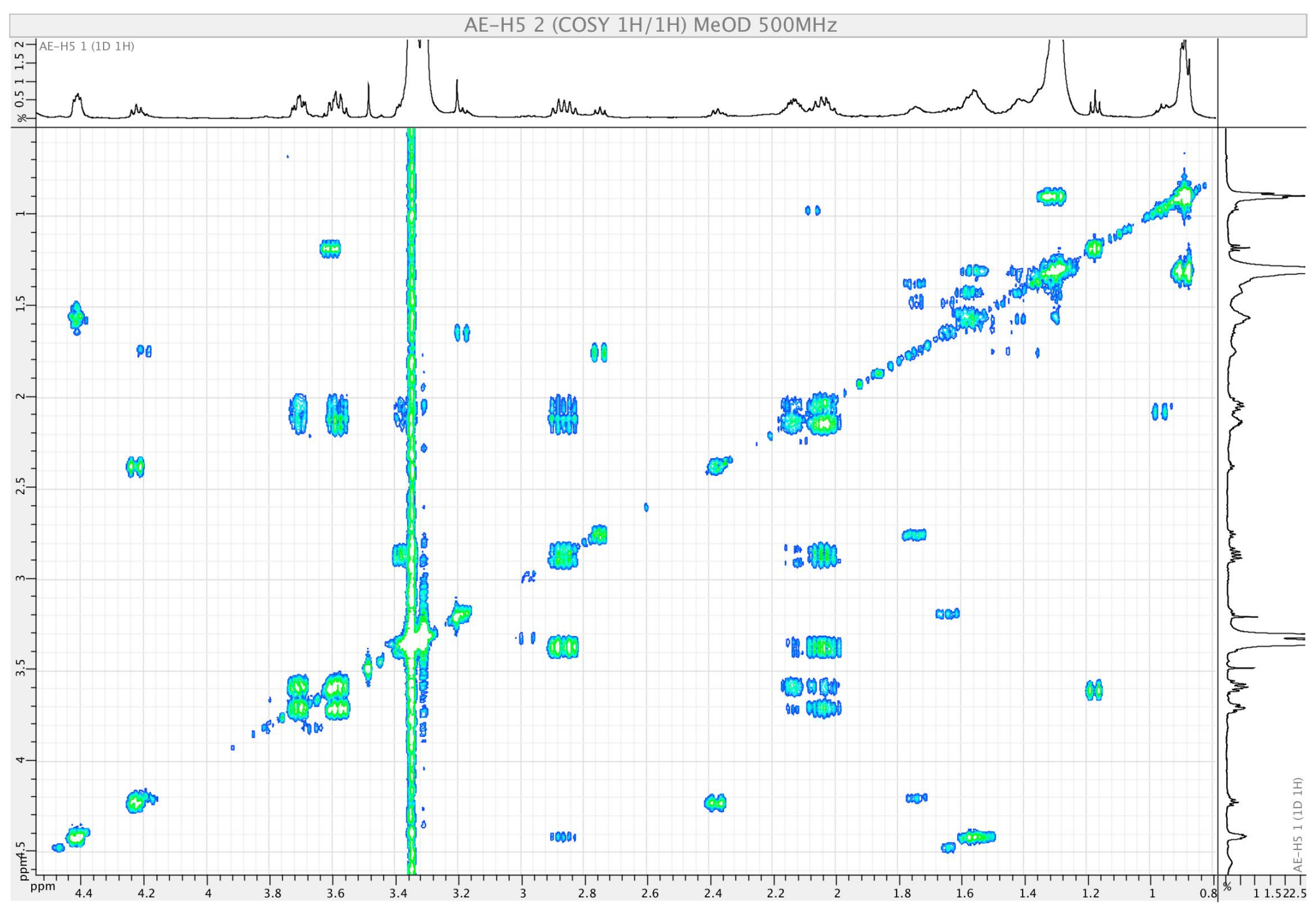


S4. HSQC NMR spectrum of monanchoradin A (1) in $\mathrm{CD}_{3} \mathrm{OD}(500 \mathrm{MHz})$.

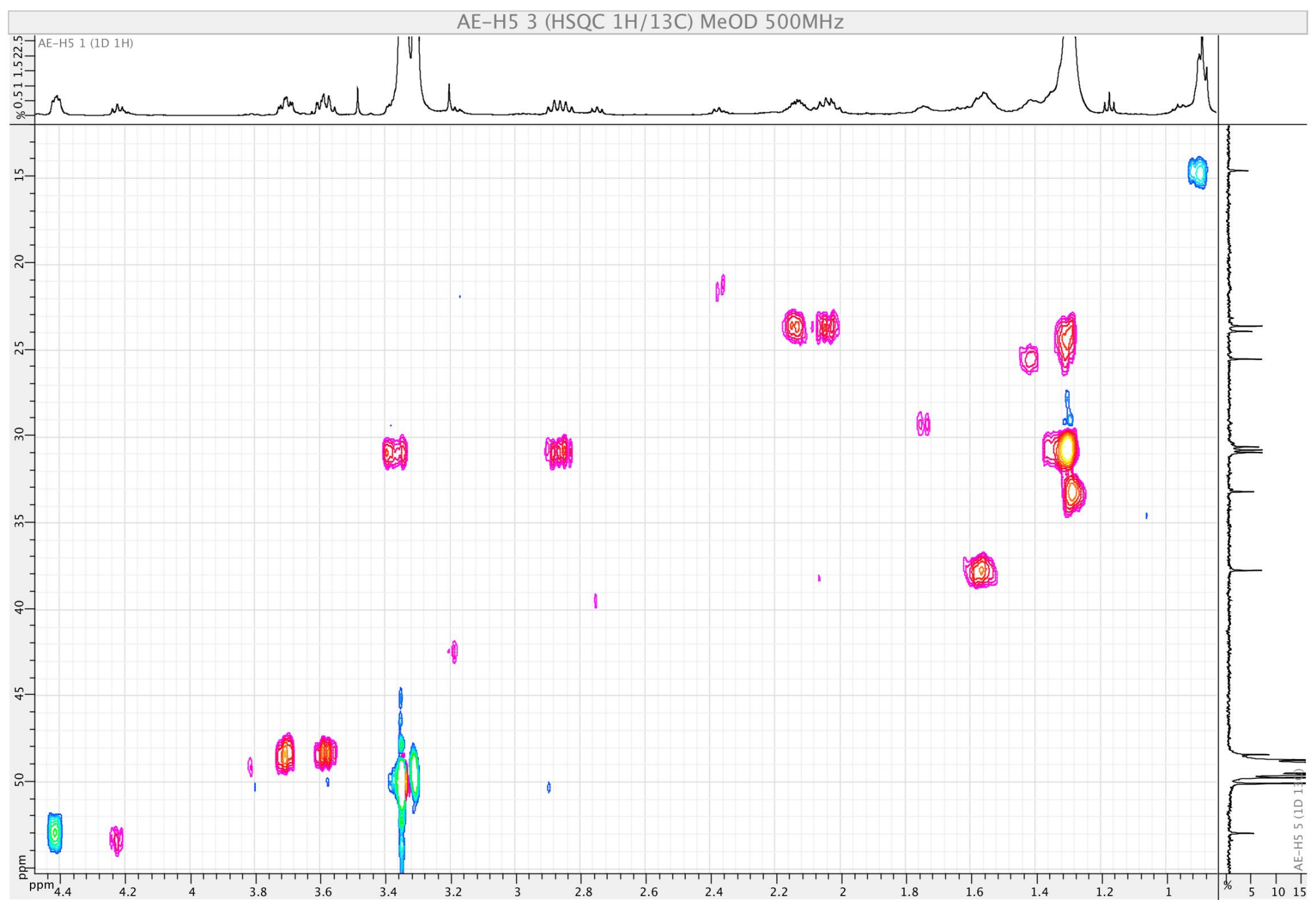


S5. ${ }^{1} \mathrm{H}-{ }^{13} \mathrm{C}$ HMBC NMR spectrum of monanchoradin $\mathrm{A}(\mathbf{1})$ in $\mathrm{CD}_{3} \mathrm{OD}(500 \mathrm{MHz})$.

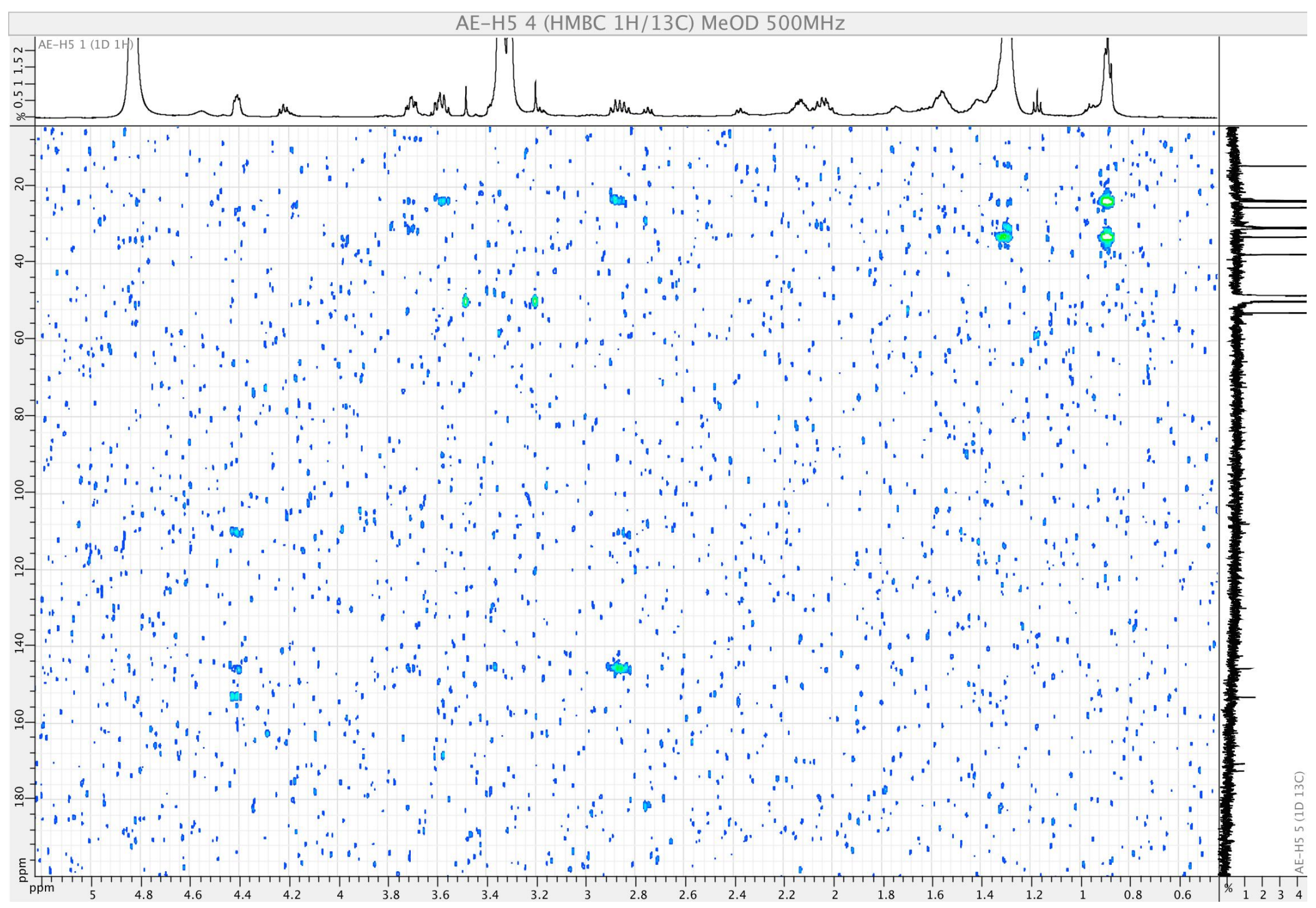


S6. UV spectrum of monanchoradin A (1) in $\mathrm{MeOH}$.

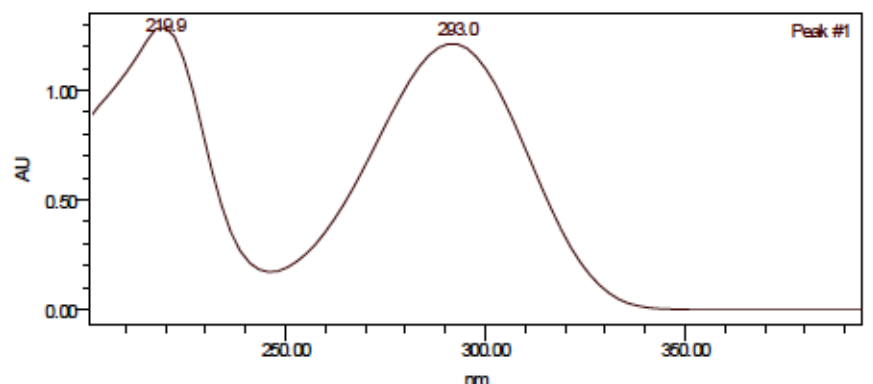

S7. IR spectrum of monanchoradin A (1).

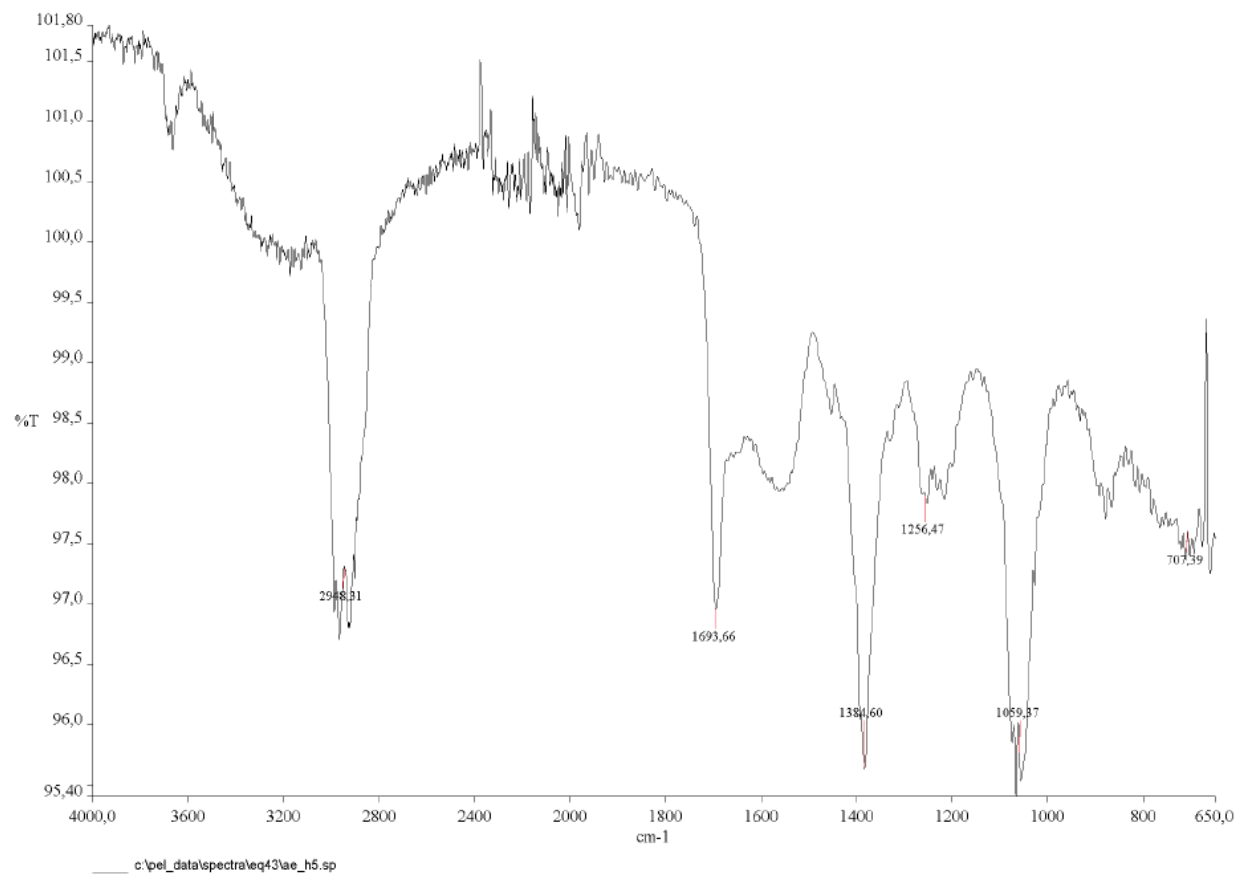


S8. ${ }^{1} \mathrm{H}$ NMR spectrum of monanchoradin B (2) in (500 MHz).

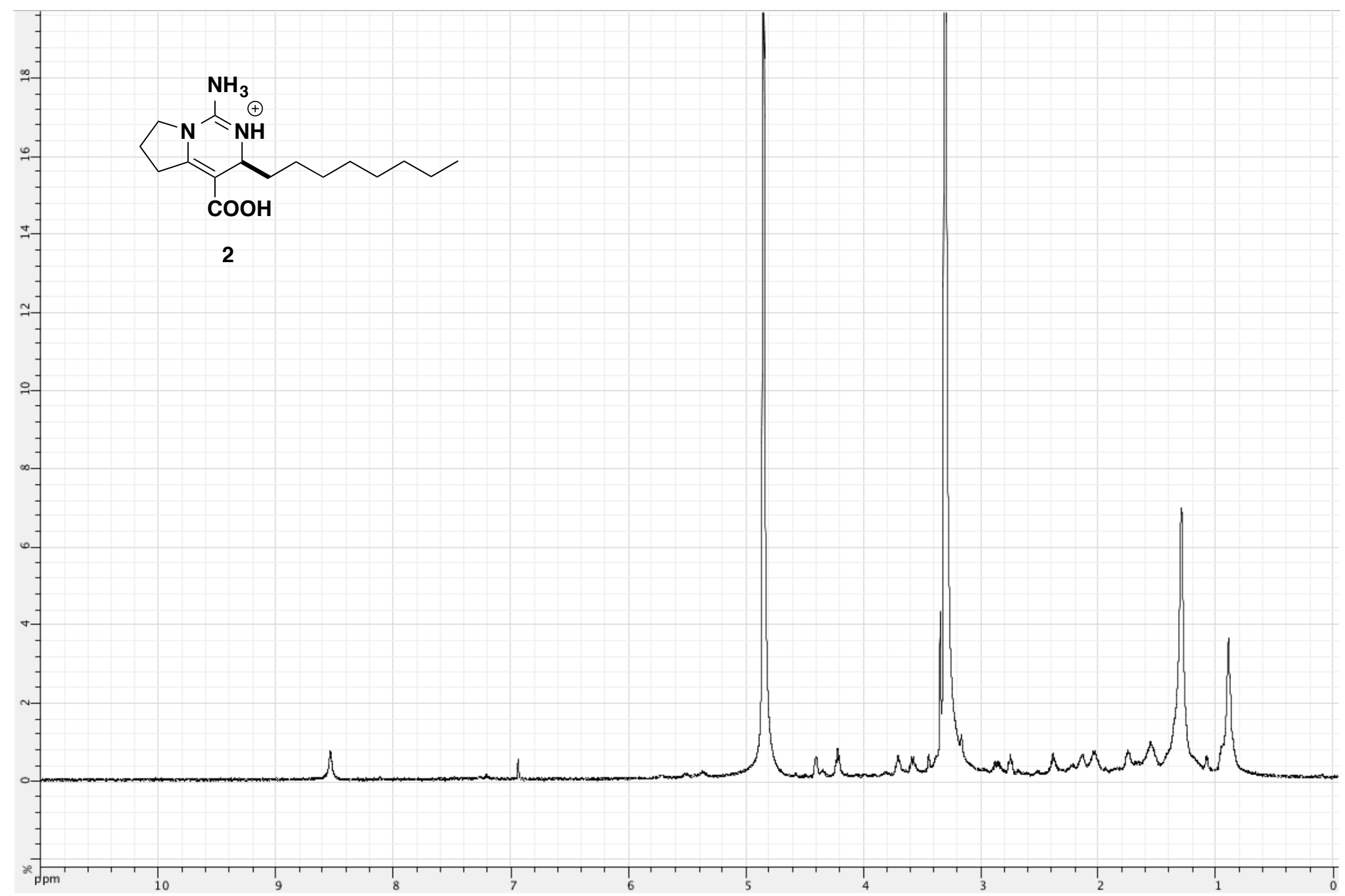


S9. ${ }^{1} \mathrm{H}$ NMR spectrum of monanchoradin C (3) in DMF-D 6 (600 MHz).

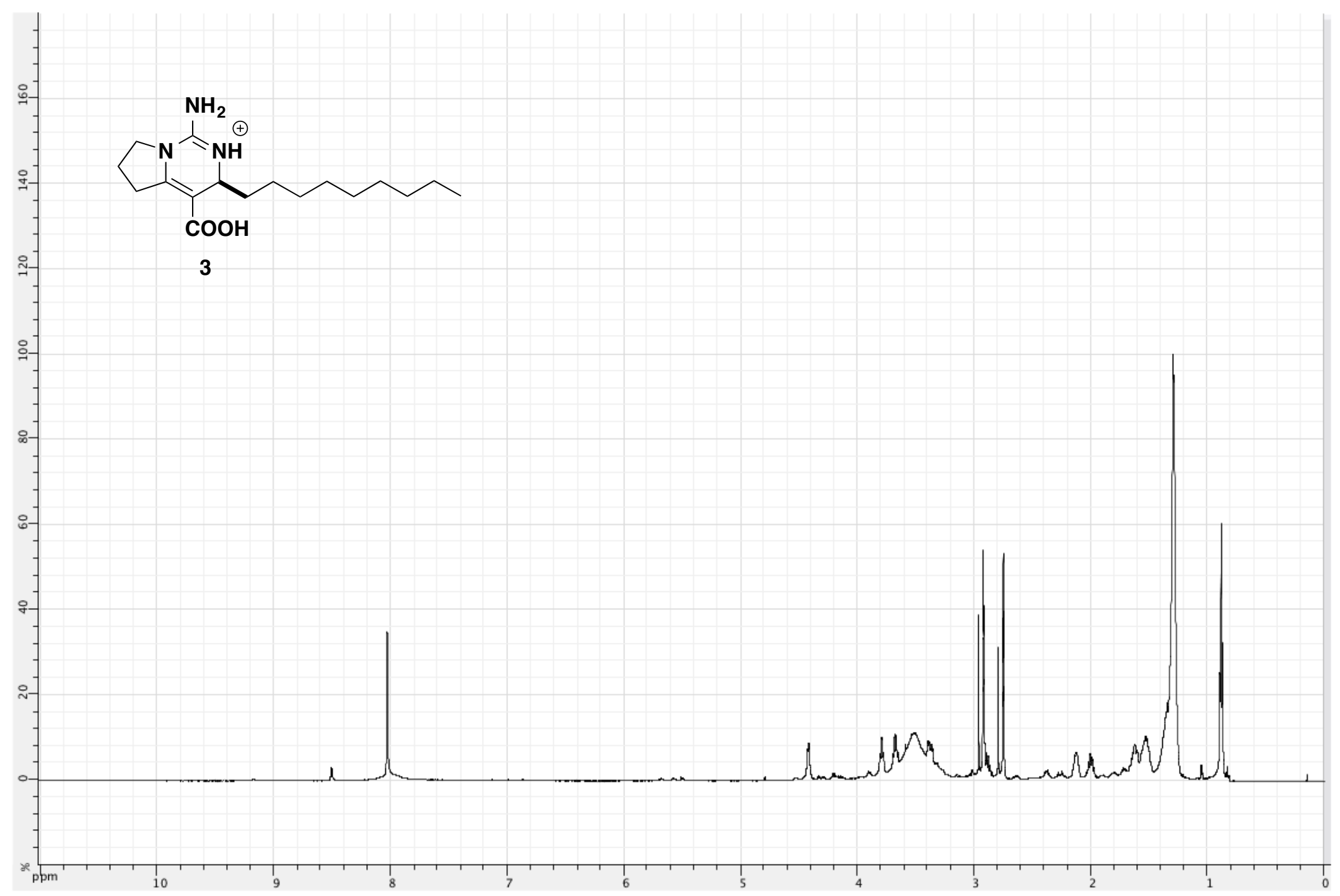


S10. ${ }^{13} \mathrm{C}$ NMR spectrum of monanchoradin C (3) in DMF (150 MHz).

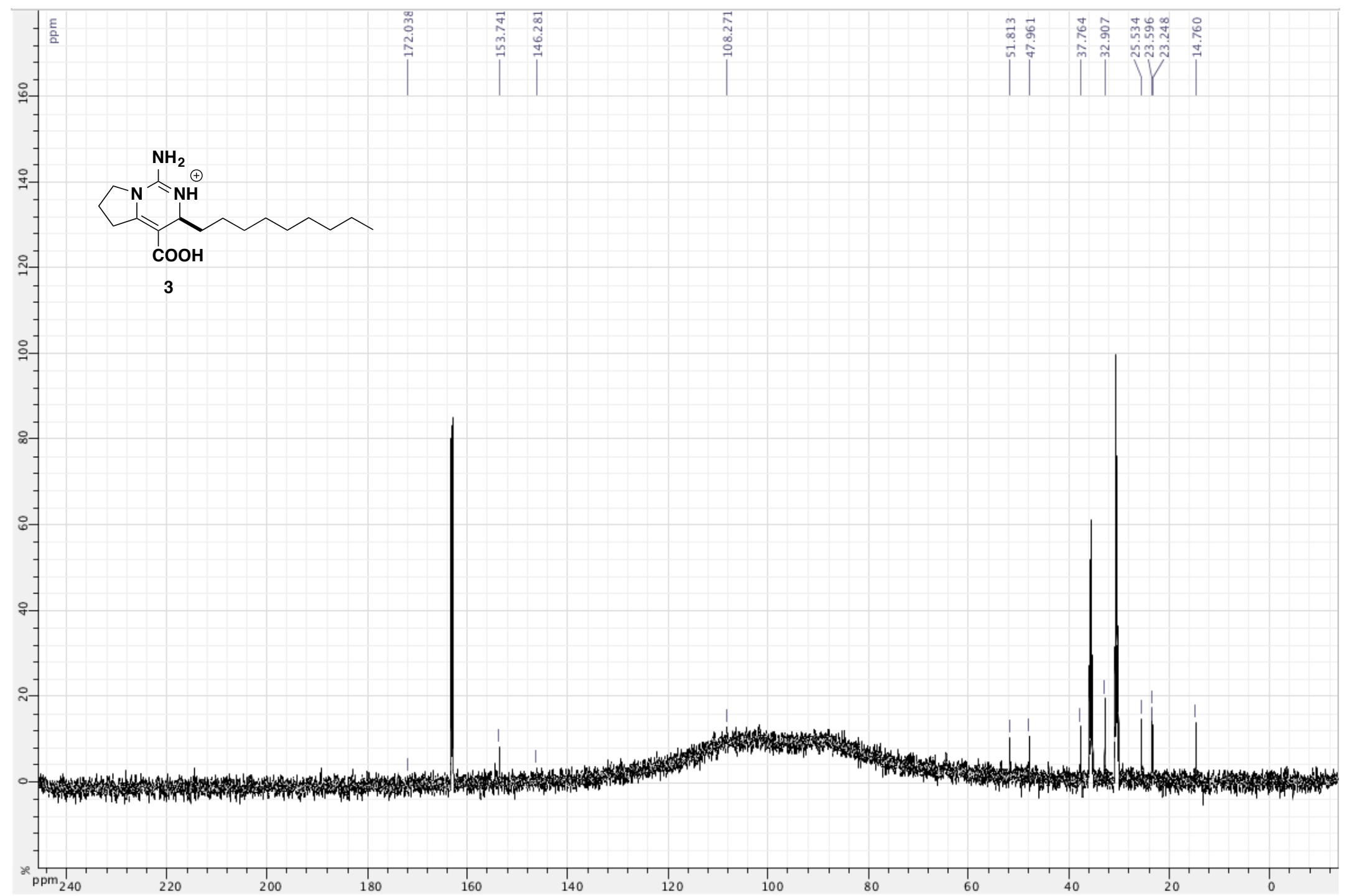


S11. ${ }^{1} \mathrm{H}-{ }^{1} \mathrm{H}$ COSY NMR spectrum of monanchoradin C (3) in DMF (600 MHz).

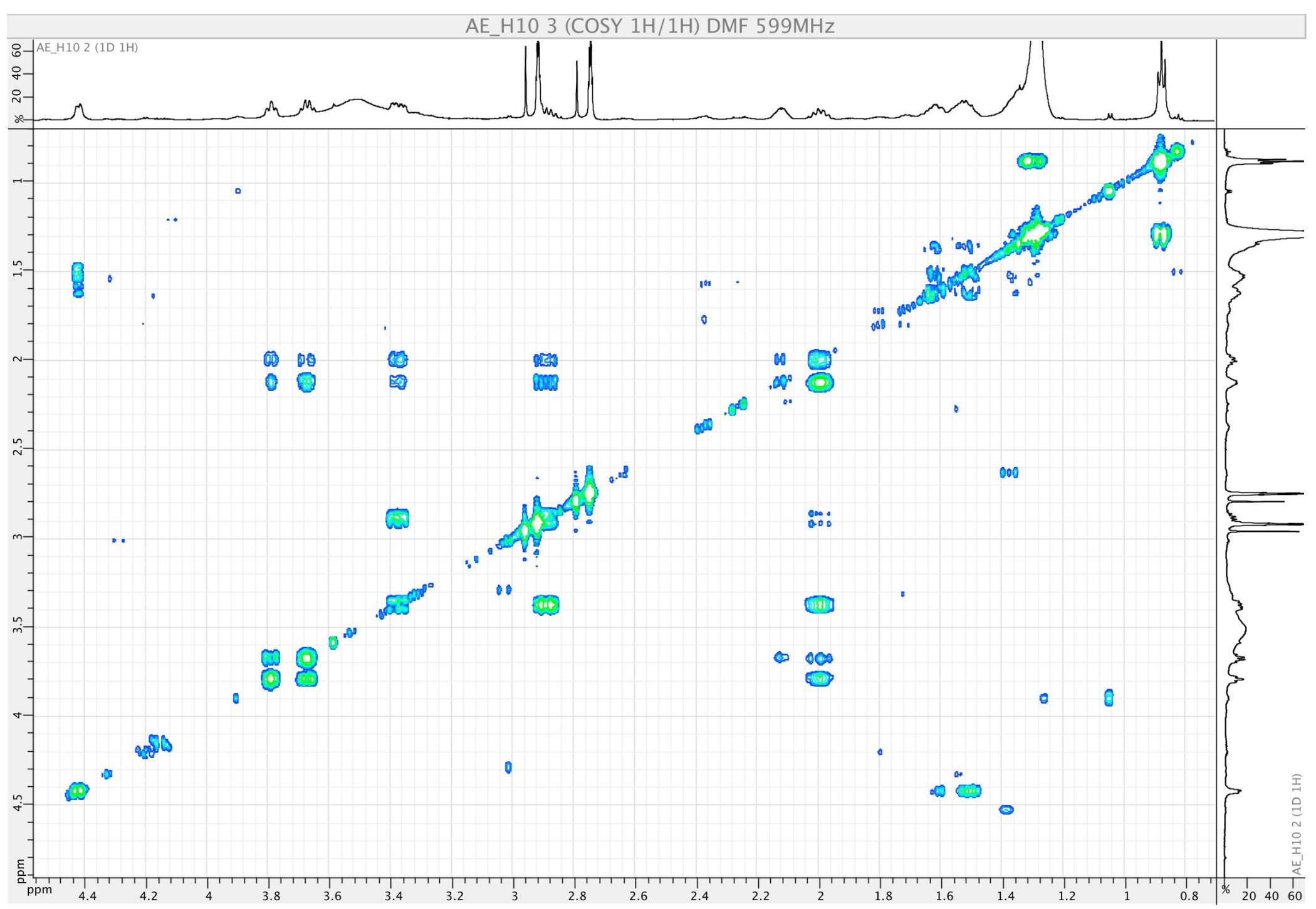


S12. HSQC NMR spectrum of monanchoradin C (3) in DMF (600 MHz).

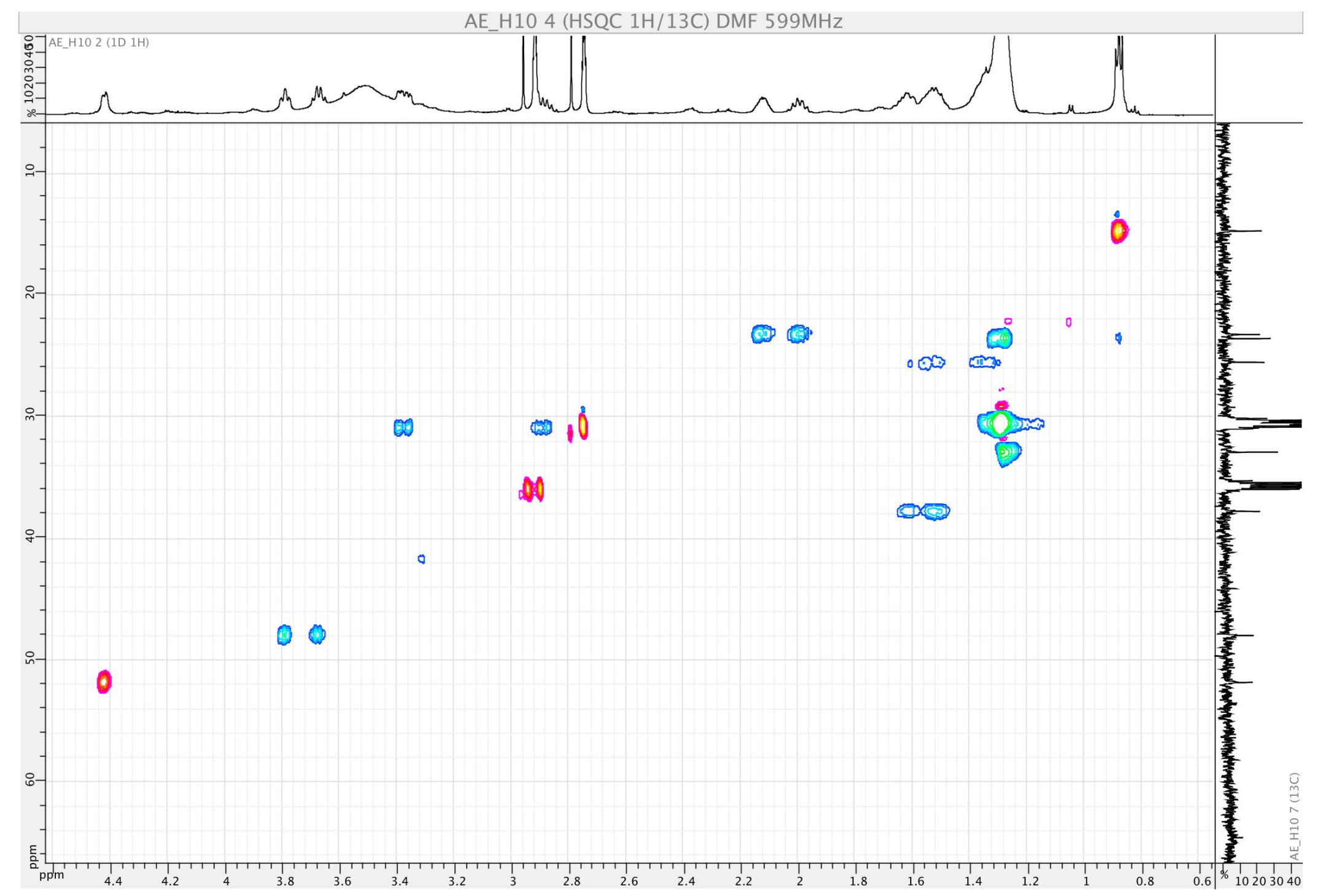


S13. ${ }^{1} \mathrm{H}-{ }^{13} \mathrm{C}$ HMBC NMR spectrum of monanchoradin C (3) in DMF (600 MHz).

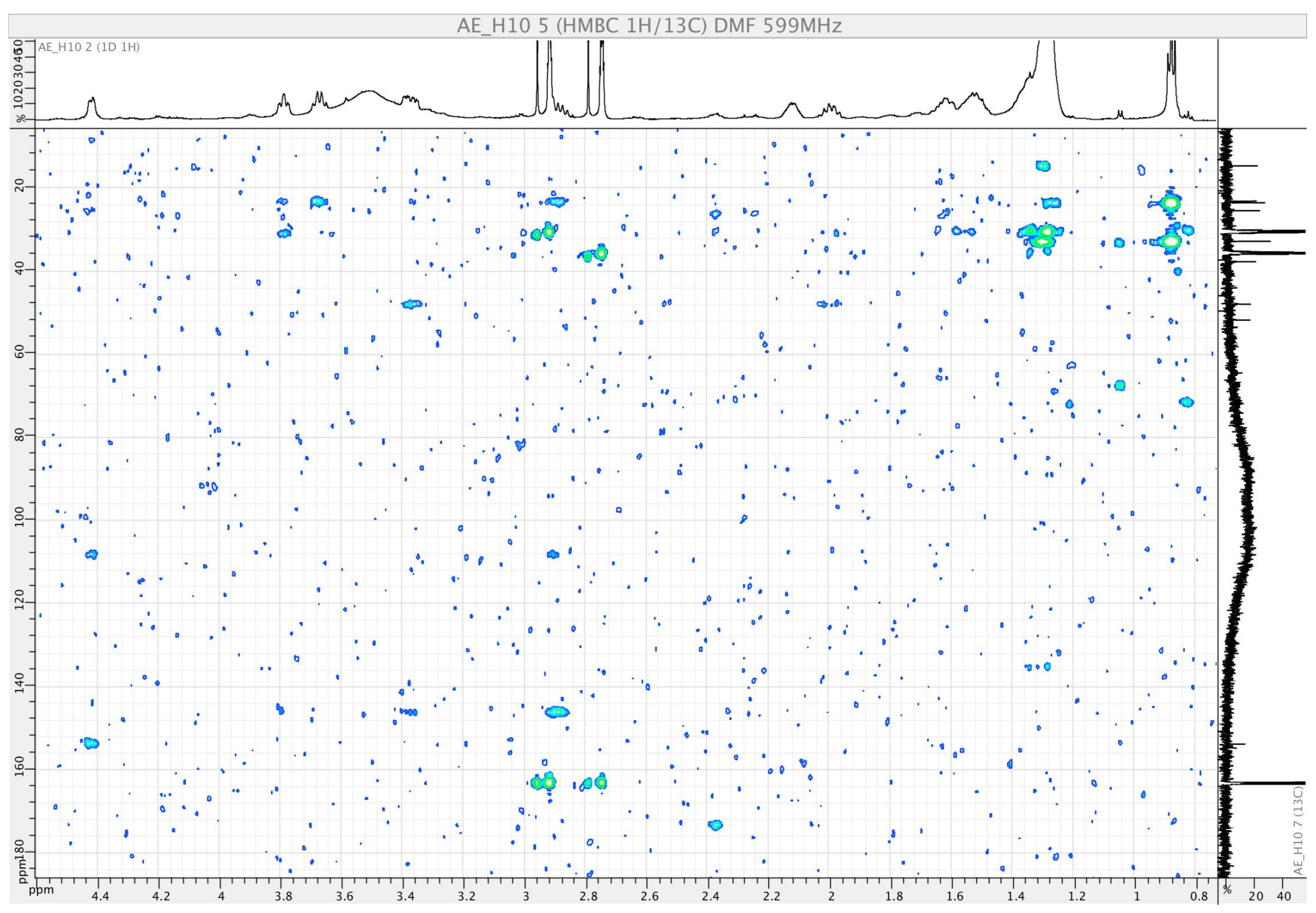


S14. UV spectrum of monanchoradin $\mathrm{C}$ (3) in $\mathrm{MeOH}$.

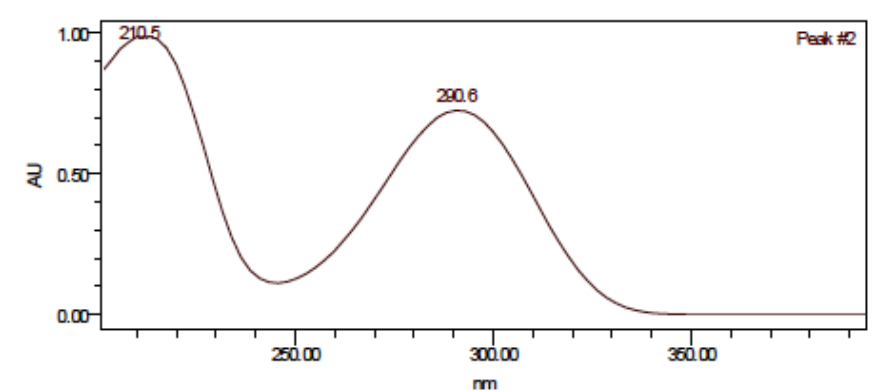

S15. IR spectrum of monanchoradin $\mathrm{C}(3)$ in $\mathrm{MeOH}$.

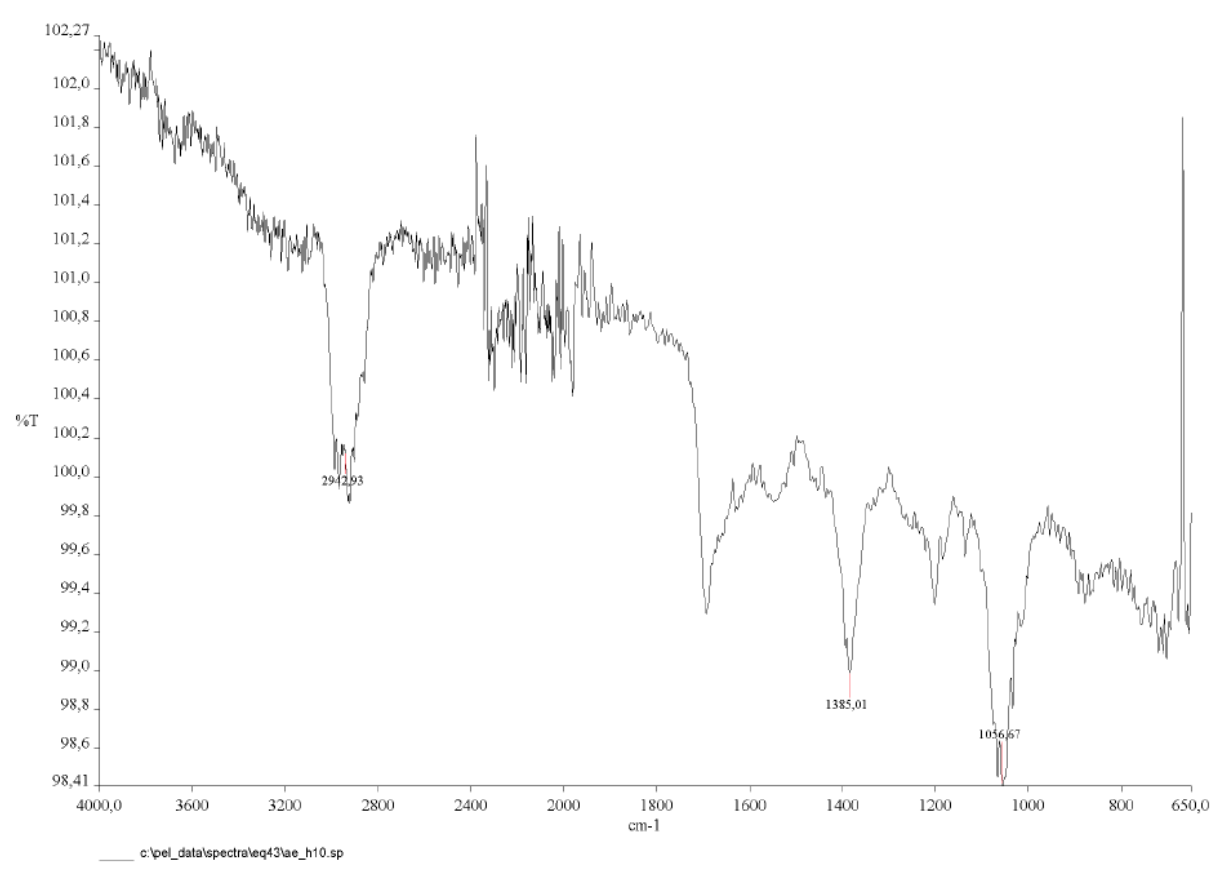


S16 ${ }^{1} \mathrm{H}$ NMR comparison for monanchoradin A (1), monanchoradin B (2) and monanchoradin C (3) in $\mathrm{CD}_{3} \mathrm{OD}(500 \mathrm{MHz})$.

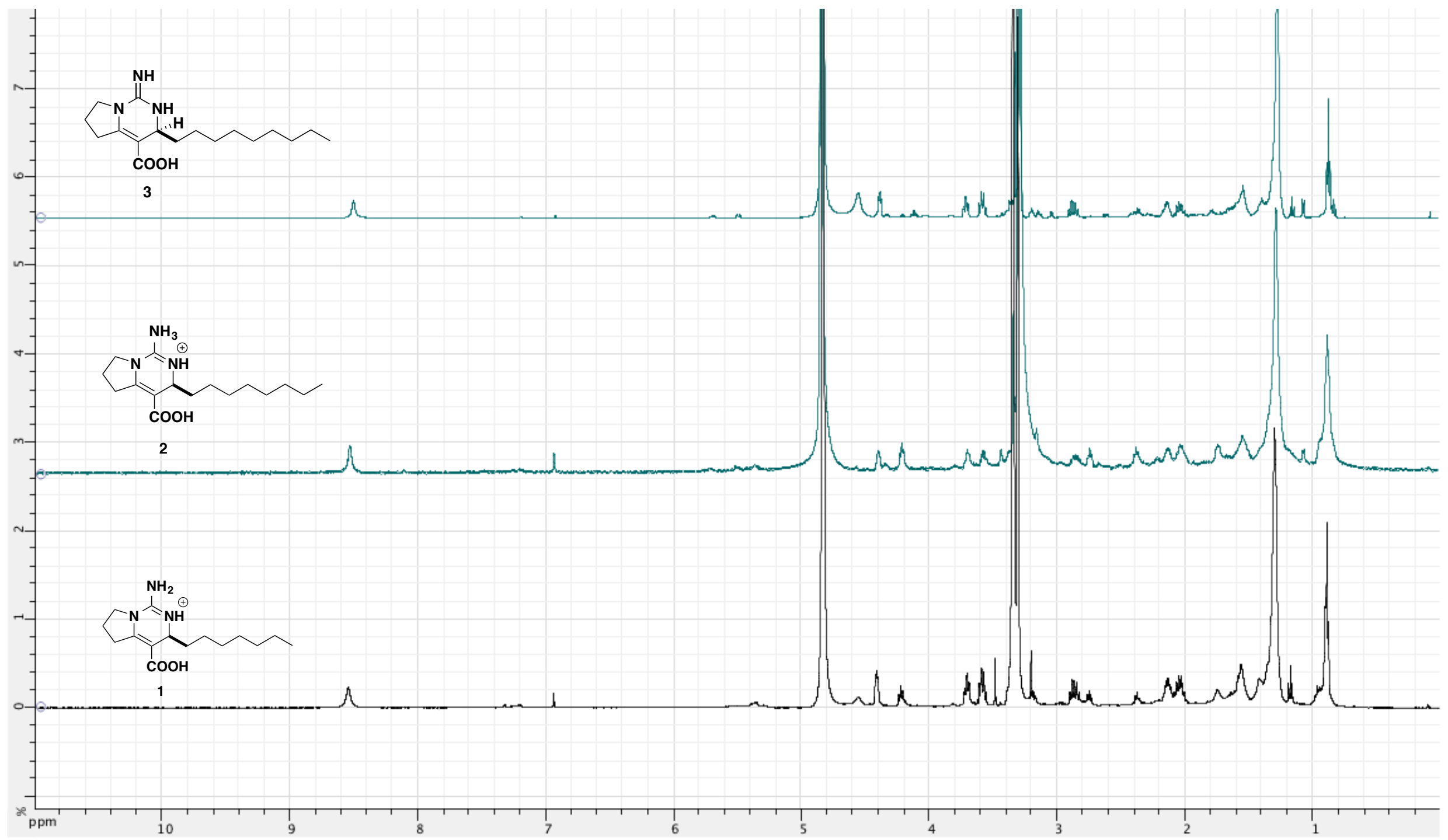


S17. ${ }^{1} \mathrm{H}$ NMR spectrum of dehydrocrambescin A2 418 (4) in $\mathrm{CD}_{3} \mathrm{OH}(500 \mathrm{MHz})$.

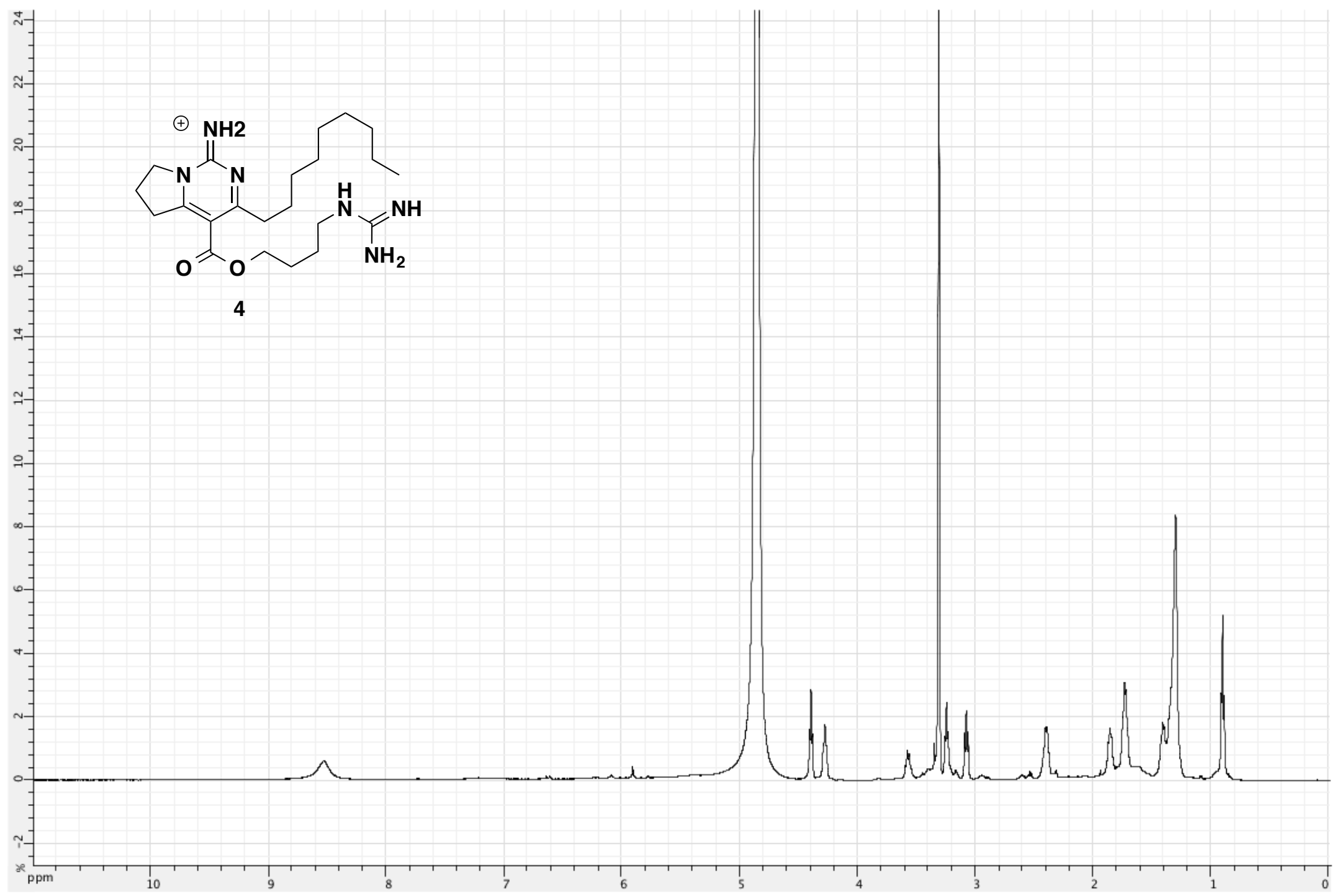


S18. ${ }^{13} \mathrm{C}$ NMR spectrum of dehydrocrambescin $\mathrm{A} 2418(4)$ in $\mathrm{CD}_{3} \mathrm{OH}(125 \mathrm{MHz})$.

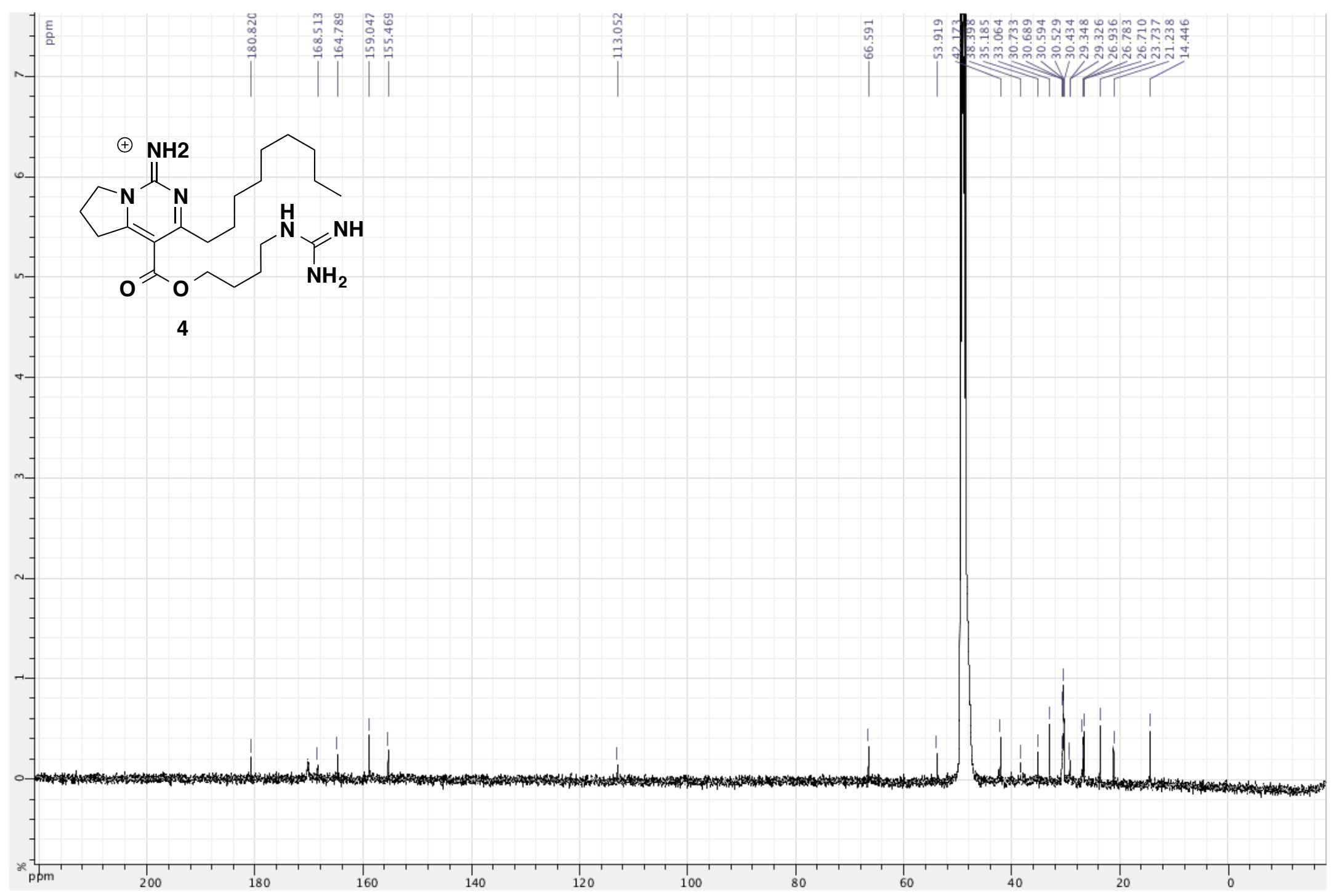


S19. ${ }^{1} \mathrm{H}-{ }^{1} \mathrm{H}$ COSY NMR spectrum of dehydrocrambescin $\mathrm{A} 2418$ (4) in $\mathrm{CD}_{3} \mathrm{OH}(500 \mathrm{MHz})$.

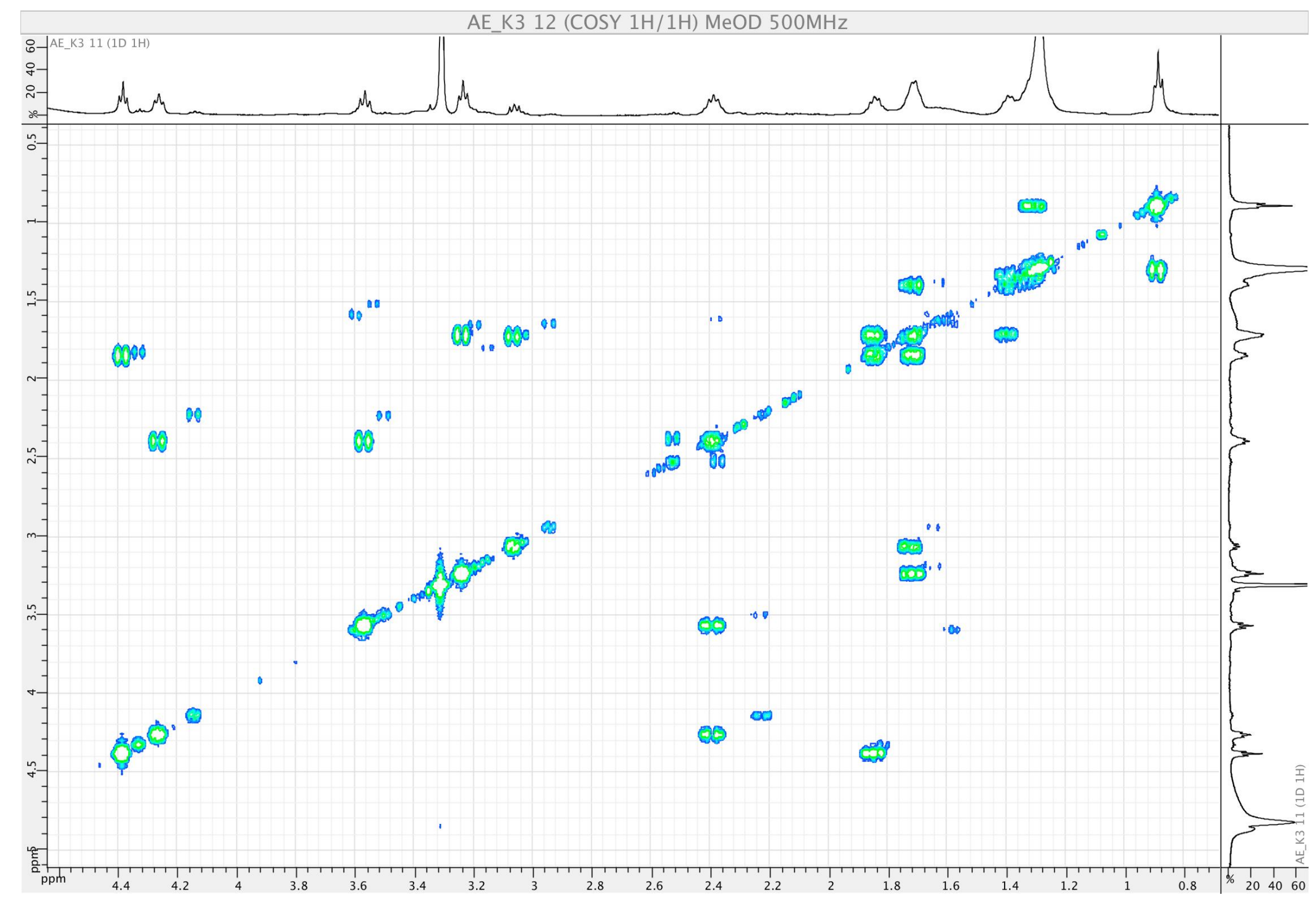


S20. HSQC NMR spectrum of dehydrocrambescin $\mathrm{A} 2418$ (4) in $\mathrm{CD}_{3} \mathrm{OH}(500 \mathrm{MHz})$.

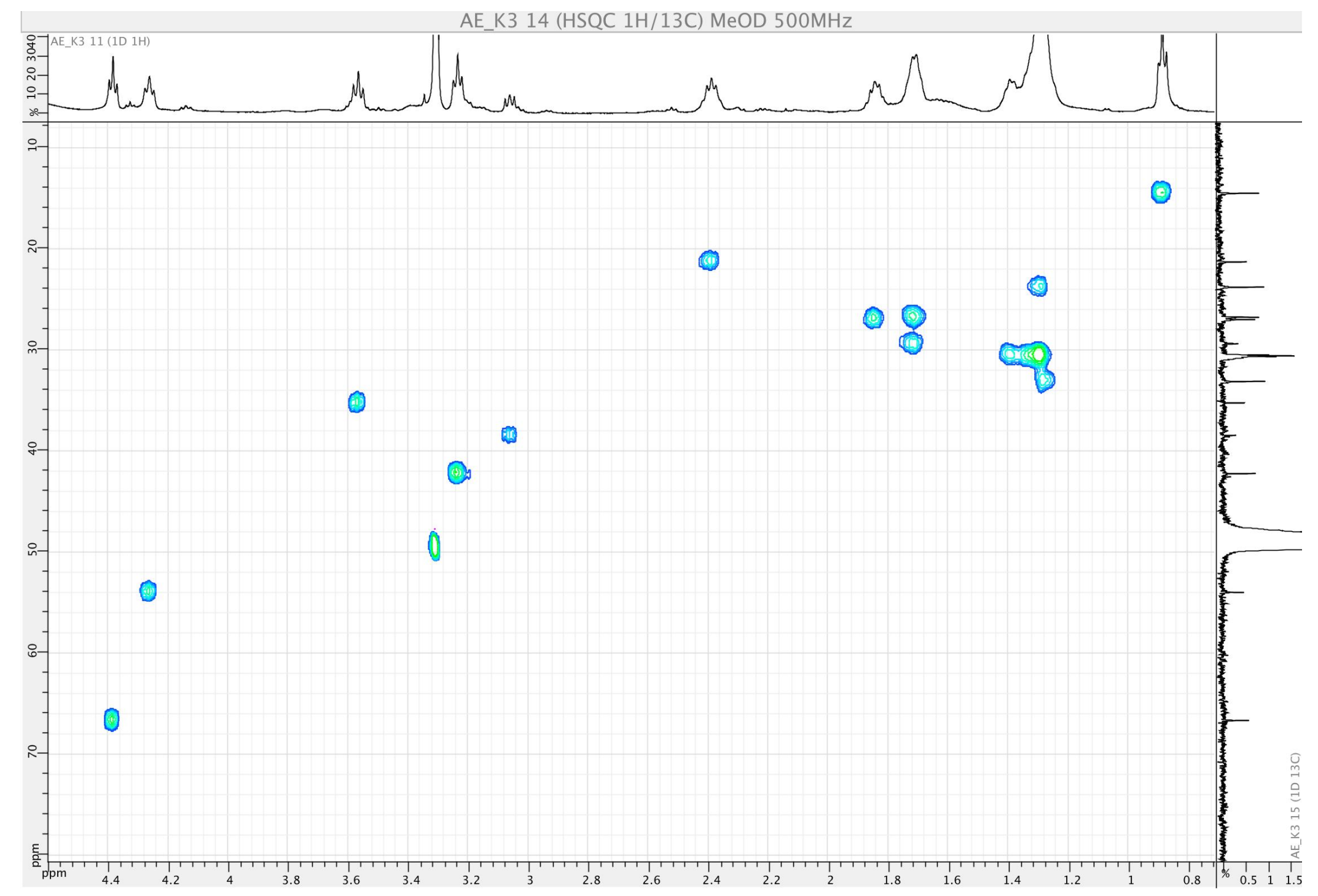


S21. ${ }^{1} \mathrm{H}-{ }^{13} \mathrm{C}$ HMBC NMR spectrum of dehydrocrambescin $\mathrm{A} 2418$ (4) in $\mathrm{CD}_{3} \mathrm{OH}(500 \mathrm{MHz})$.

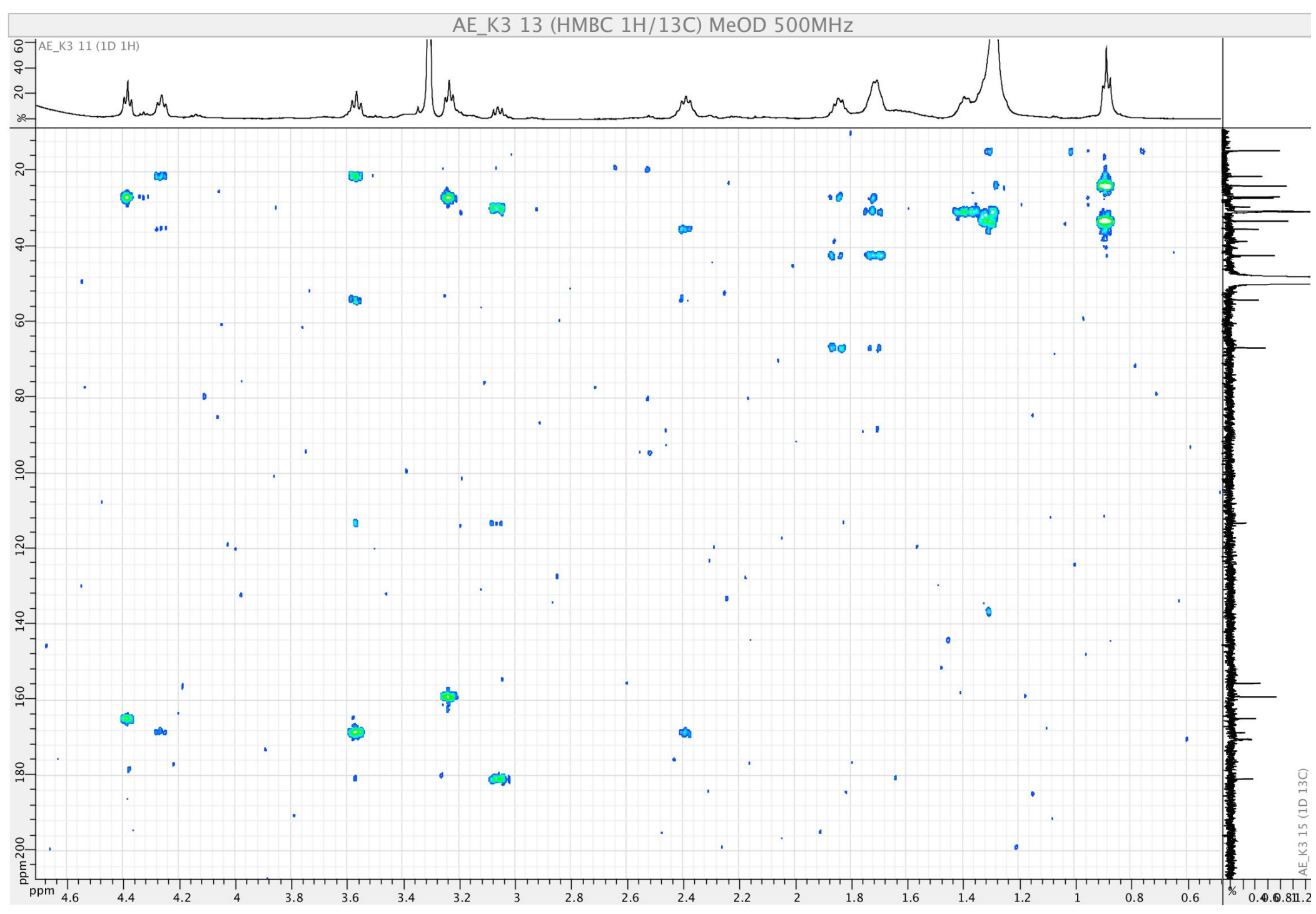


S22. UV spectrum of dehydrocrambescin A2 418 (4) in $\mathrm{MeOH}$.

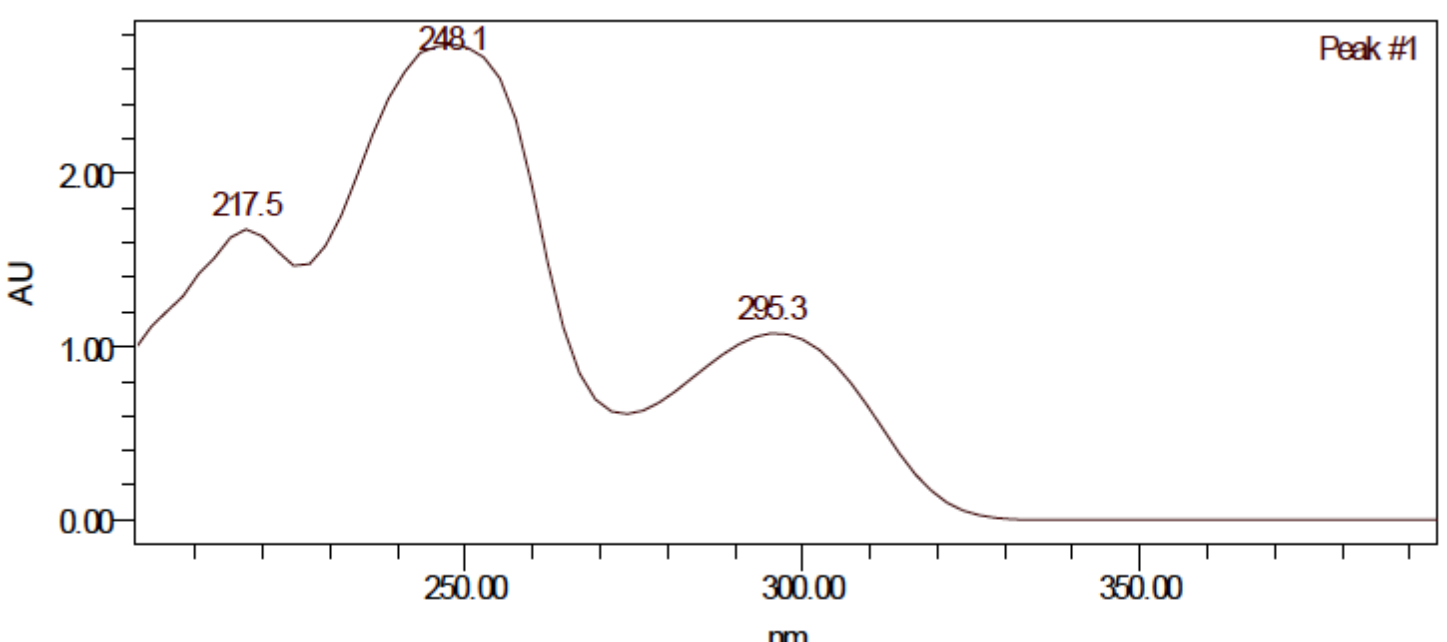

S23. IR spectrum of dehydrocrambescin A2 418 (4). 


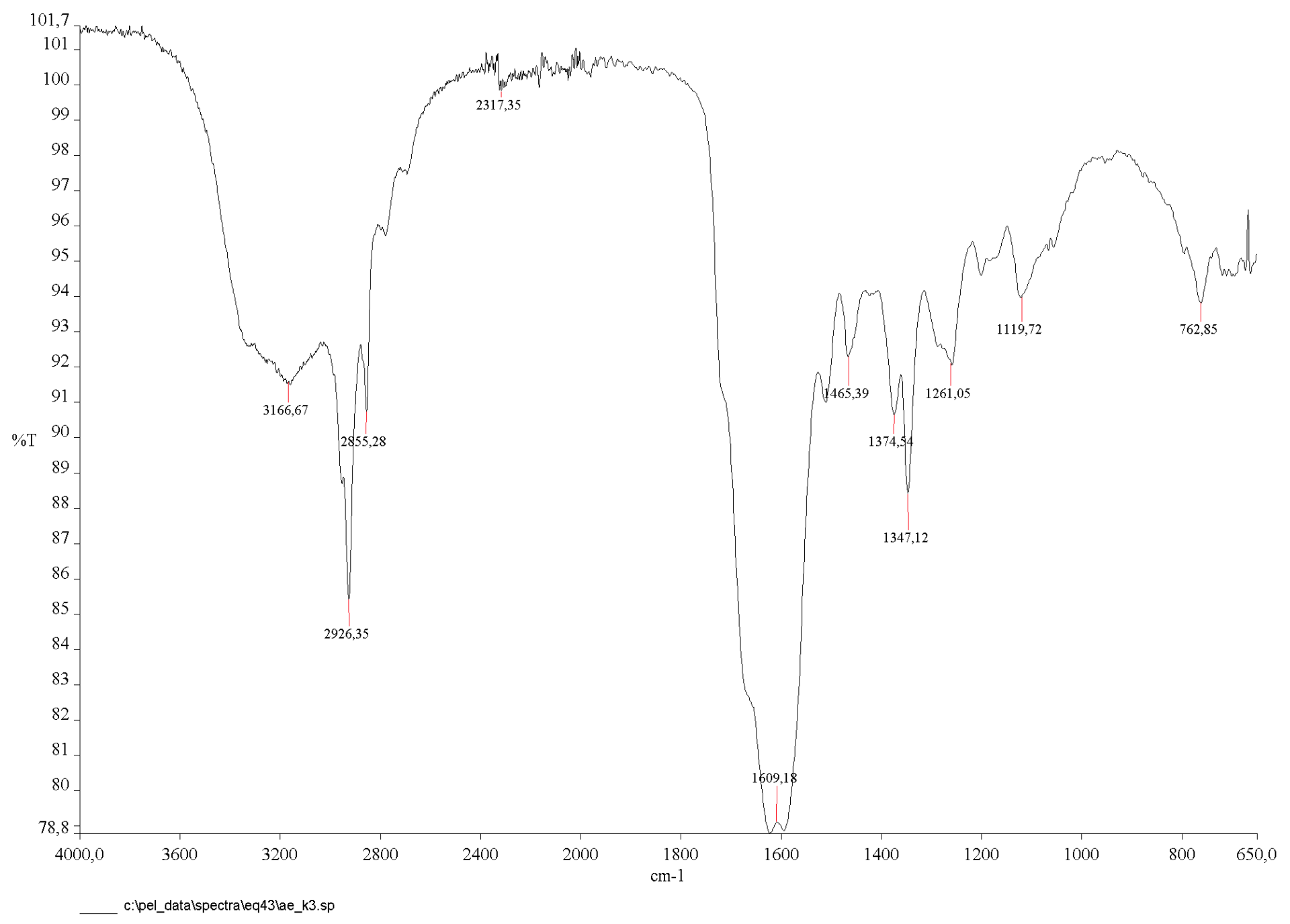

S24. Variation of ${ }^{1} \mathrm{H}$ NMR of dehydrocrambescin A2 418 (4) with time in $\mathrm{CD}_{3} \mathrm{OD}(500 \mathrm{MHz})$. 


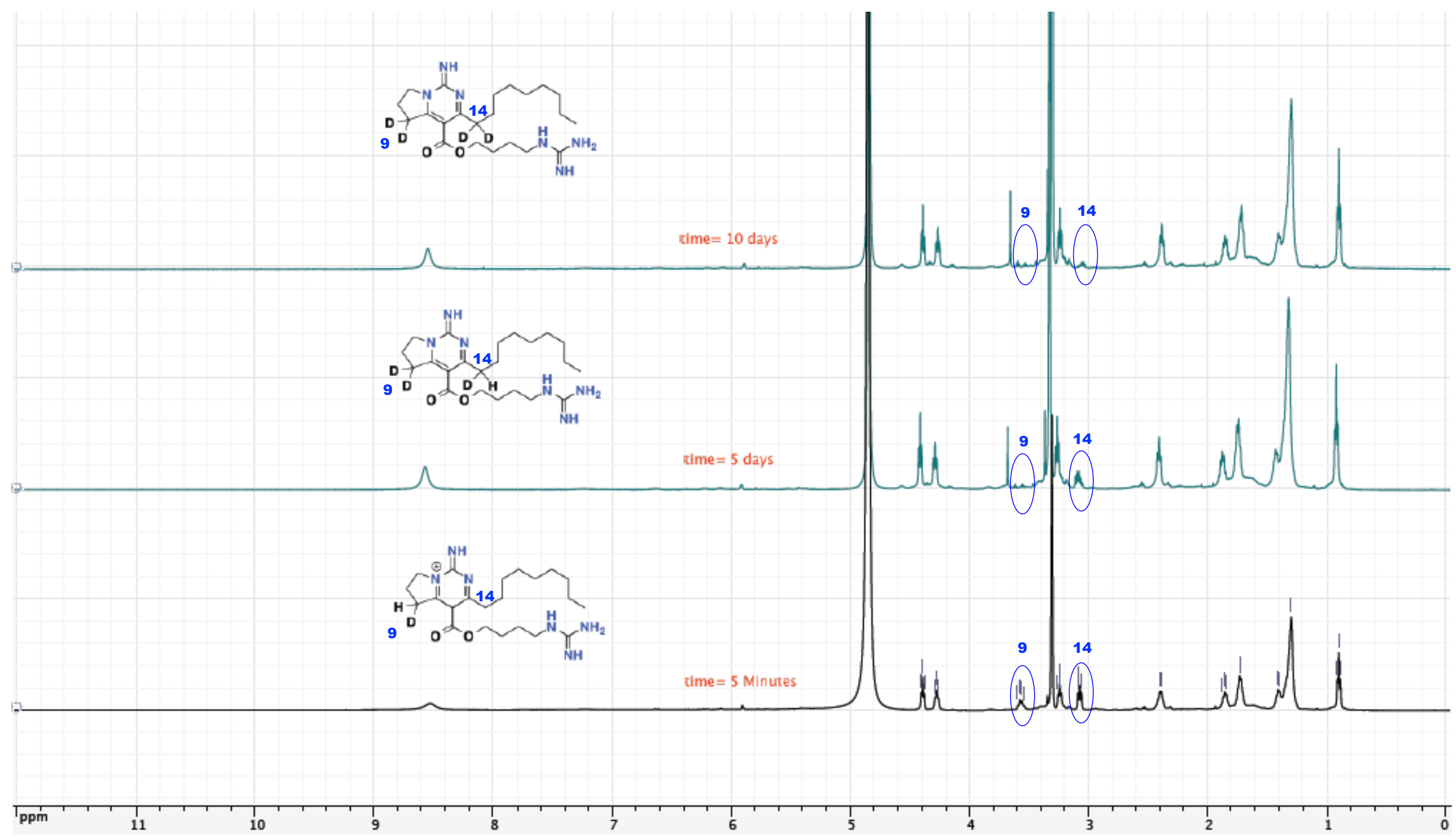

S25. Dehydrocrambescin A2 418 (4) through the exchange of allylic hydrogen atoms with deuterium in $\mathrm{CD}_{3} \mathrm{OD}(500 \mathrm{MHz})$. 


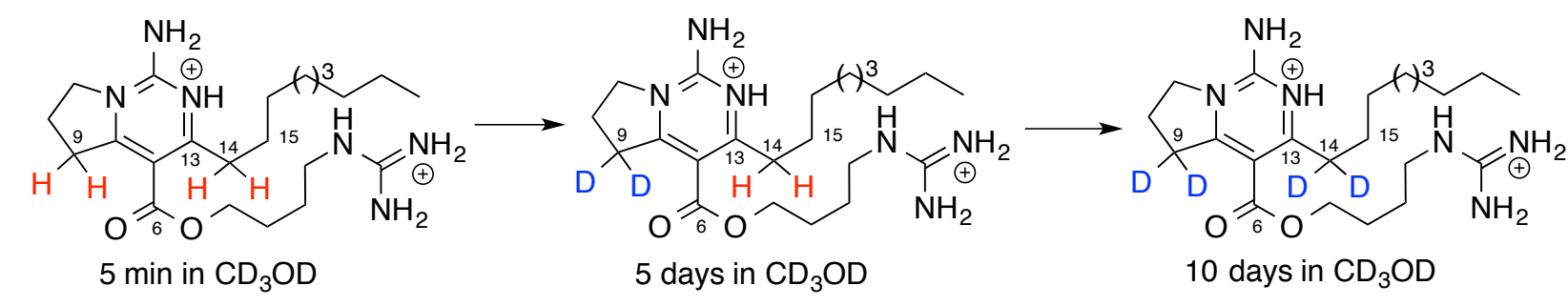

4

S26. ${ }^{1} \mathrm{H}$ NMR spectrum of crambescidin 786 (5) in $\mathrm{CD}_{3} \mathrm{OD}(500 \mathrm{MHz})$. 


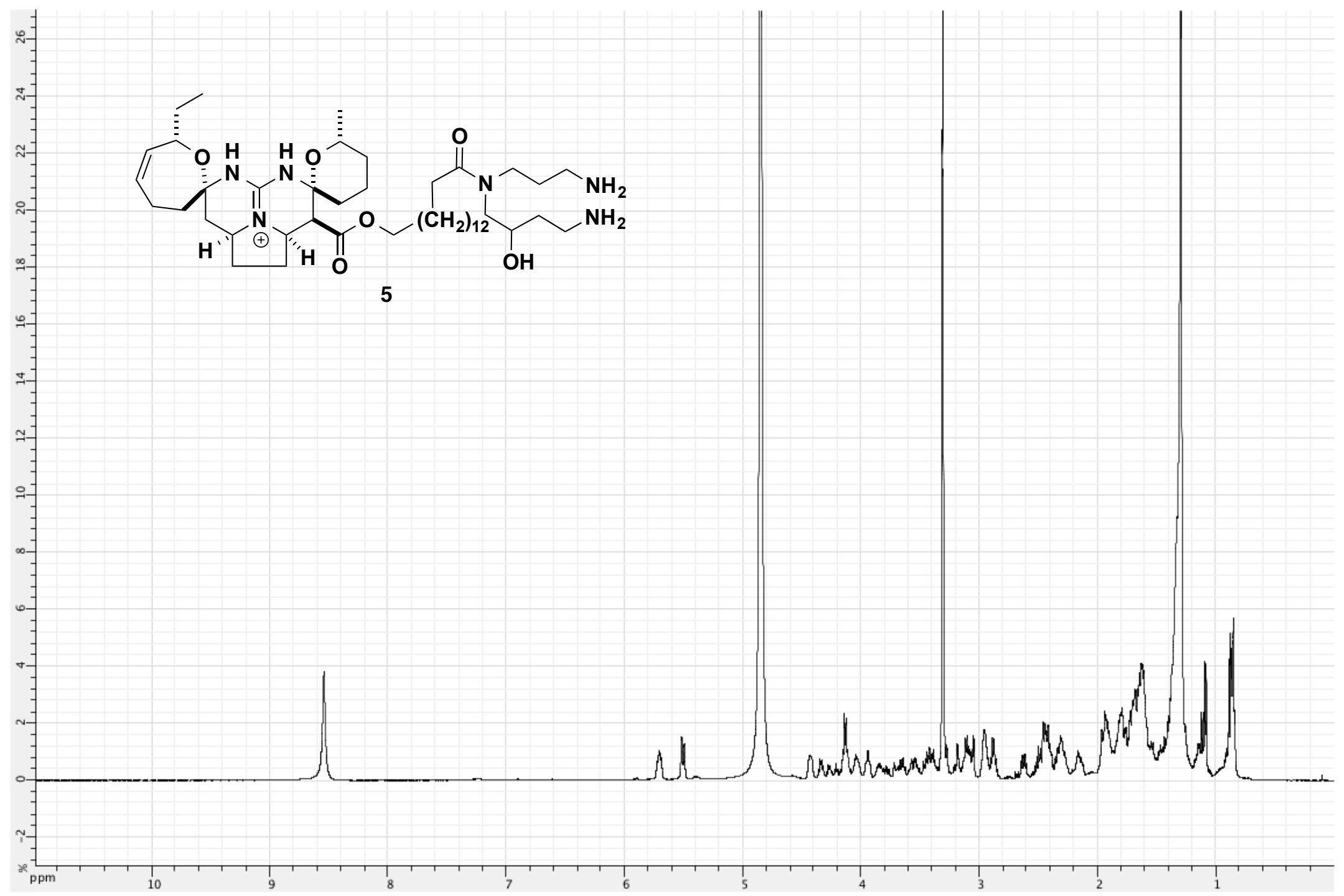

S27. ${ }^{13} \mathrm{C}$ NMR spectrum of crambescidin $786(5)$ in $\mathrm{CD}_{3} \mathrm{OD}(125 \mathrm{MHz})$. 


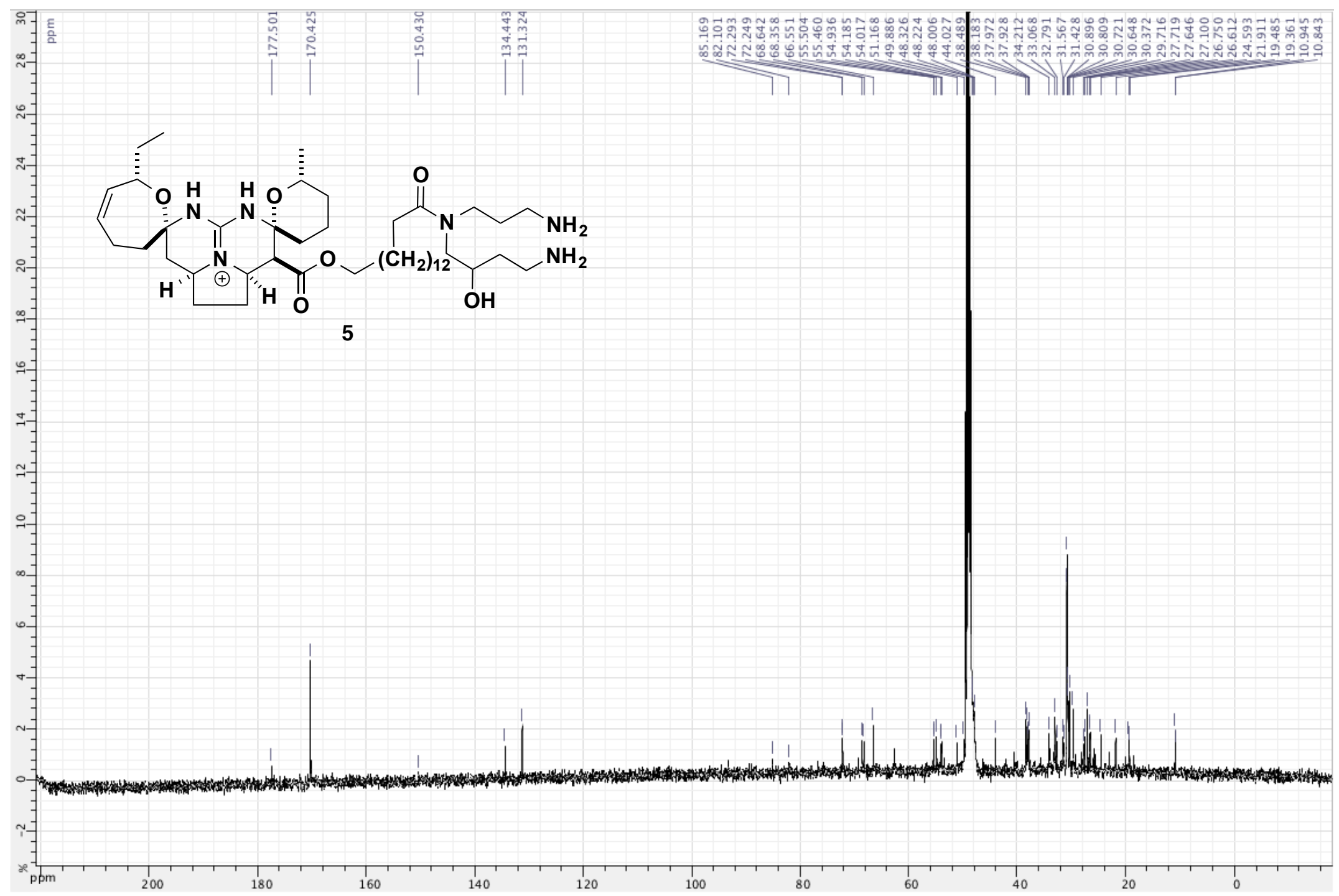

S28. ${ }^{1} \mathrm{H}-{ }^{1} \mathrm{H}$ COSY NMR spectrum of crambescidin $786(5)$ in $\mathrm{CD}_{3} \mathrm{OD}(500 \mathrm{MHz})$. 


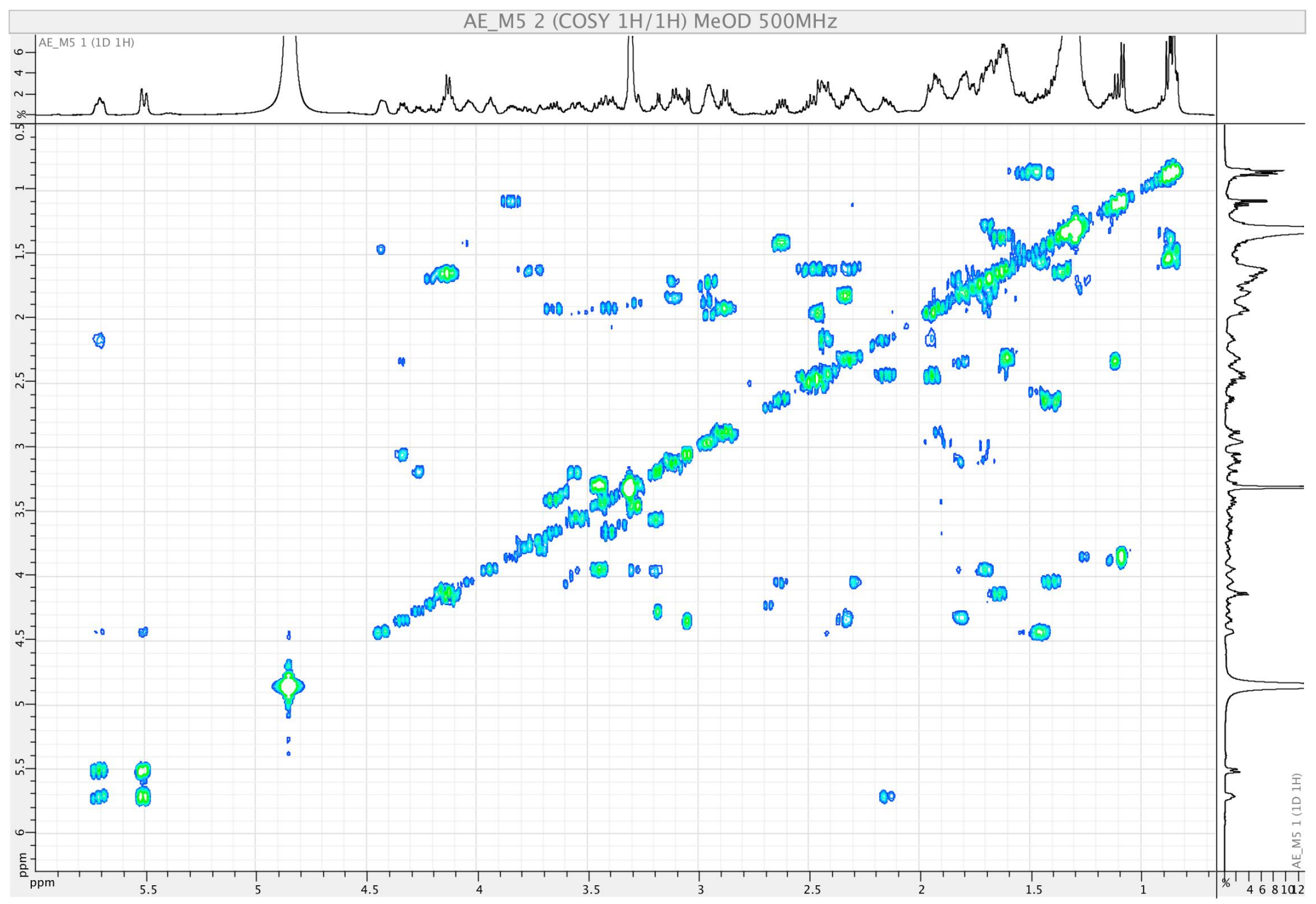

S29. HSQC NMR spectrum of crambescidin 786 (5) in $\mathrm{CD}_{3} \mathrm{OD}(500 \mathrm{MHz})$. 


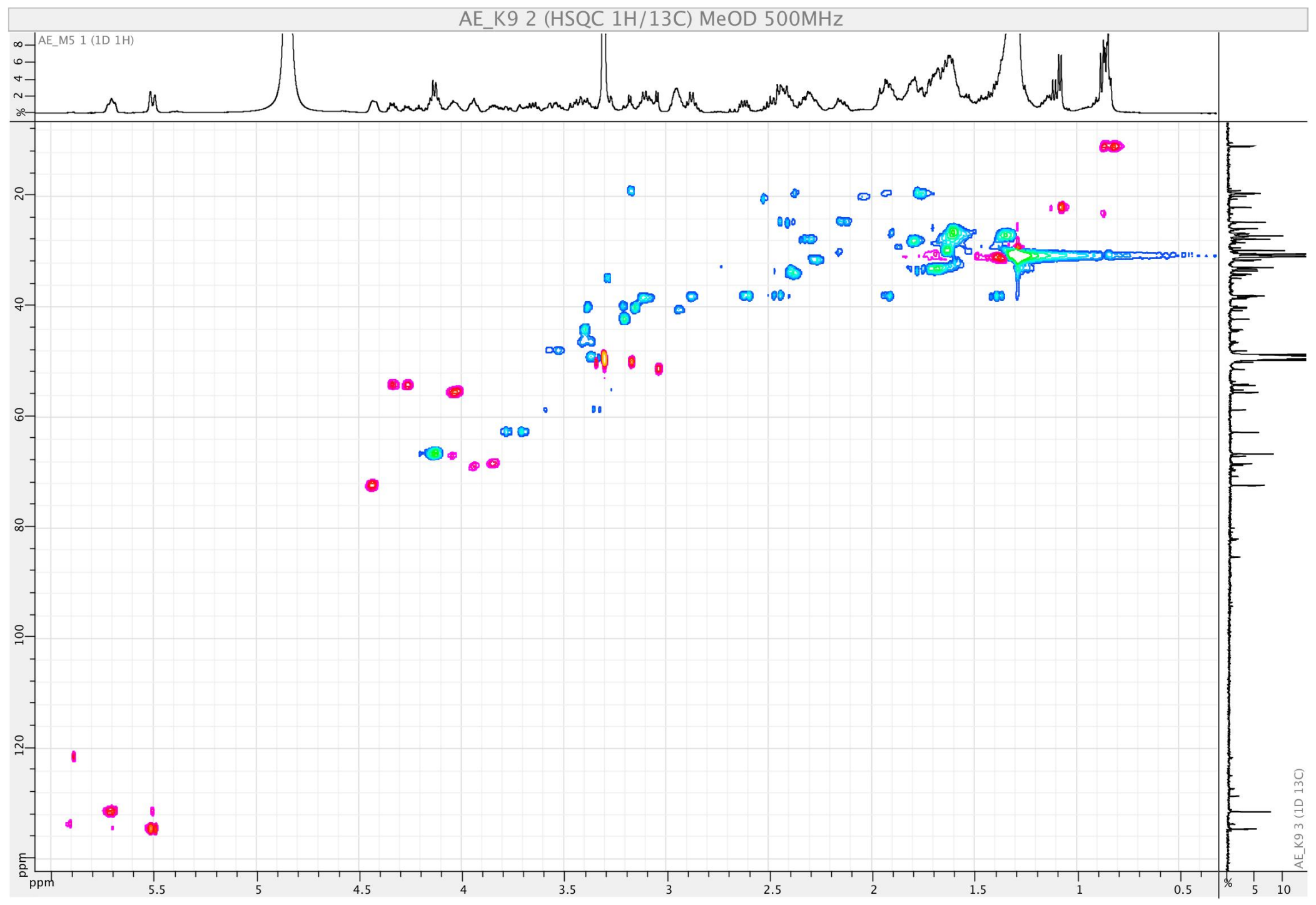

S30. UV spectrum of crambescidin 786 (5) in $\mathrm{MeOH}$. 


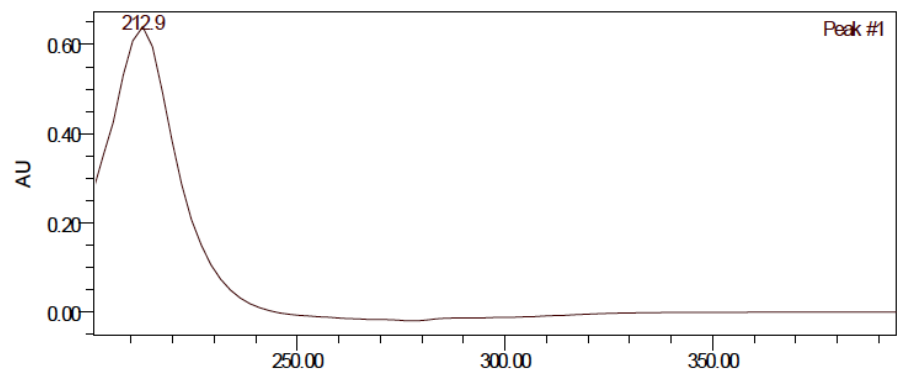

S31. IR spectrum of crambescidin 786 (5).

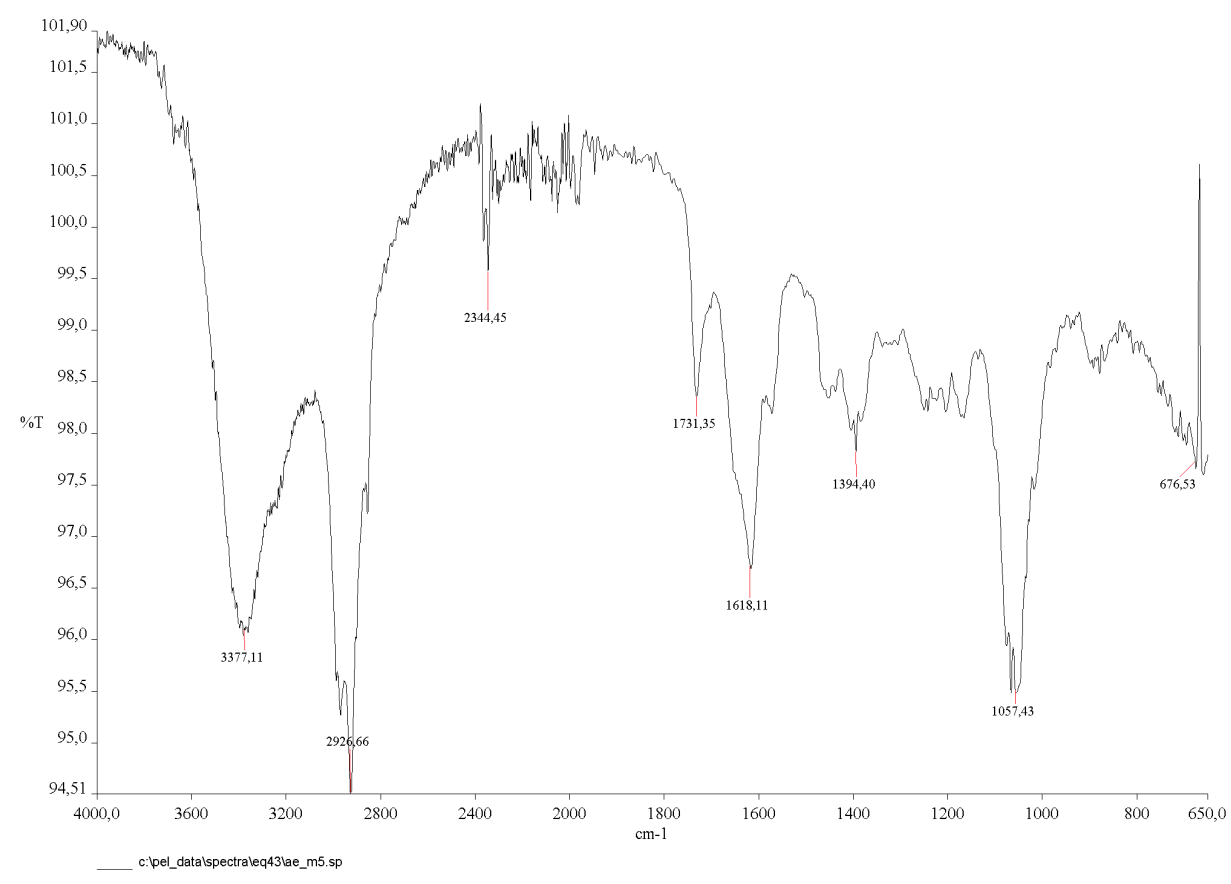

S32. ${ }^{1} \mathrm{H}$ NMR spectrum of crambescidin 814 (6) in $\mathrm{CD}_{3} \mathrm{OD}(500 \mathrm{MHz})$. 


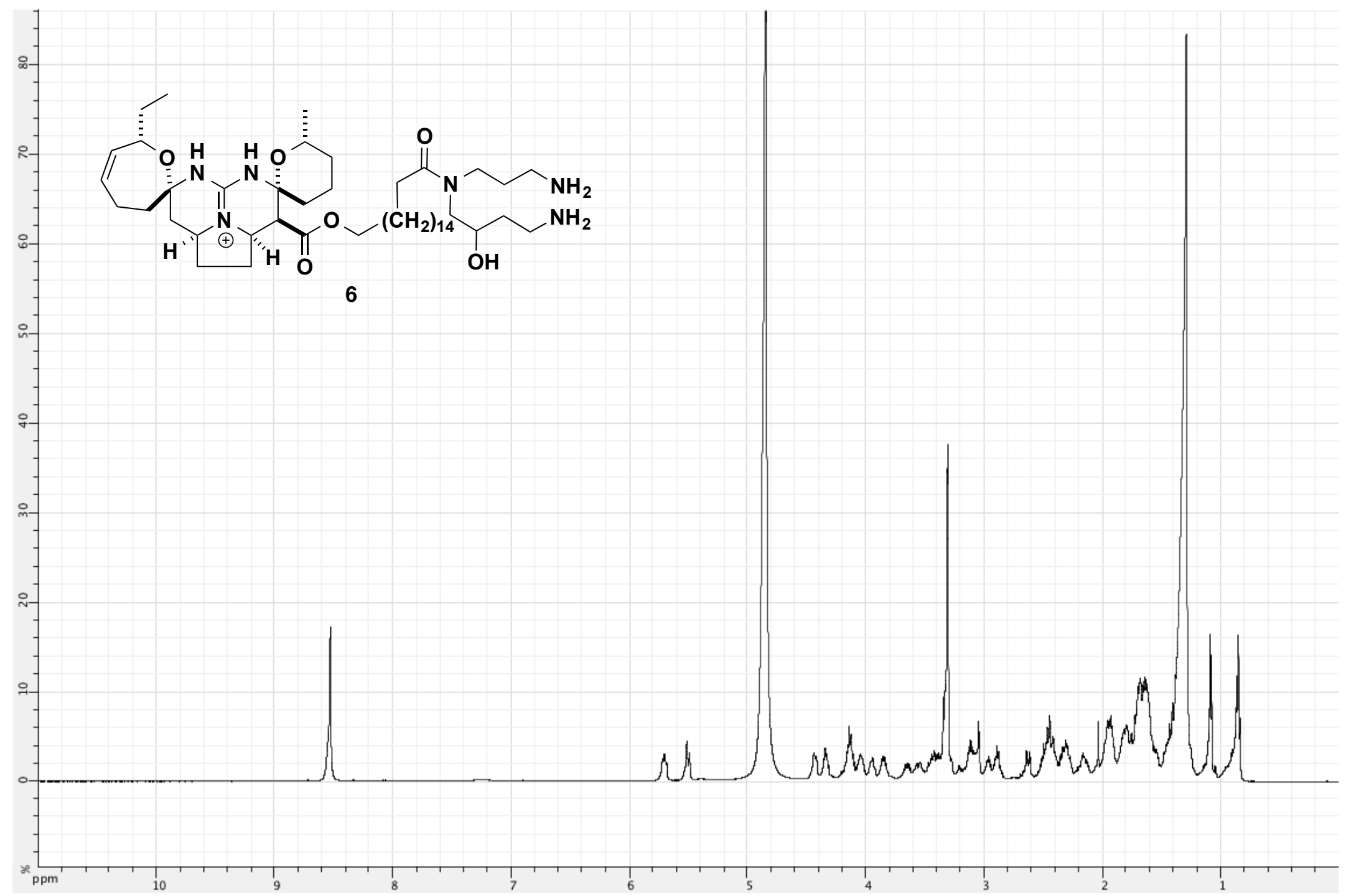

S33. ${ }^{13} \mathrm{C}$ NMR spectrum of crambescidin $814(6)$ in $\mathrm{CD}_{3} \mathrm{OD}(125 \mathrm{MHz})$. 


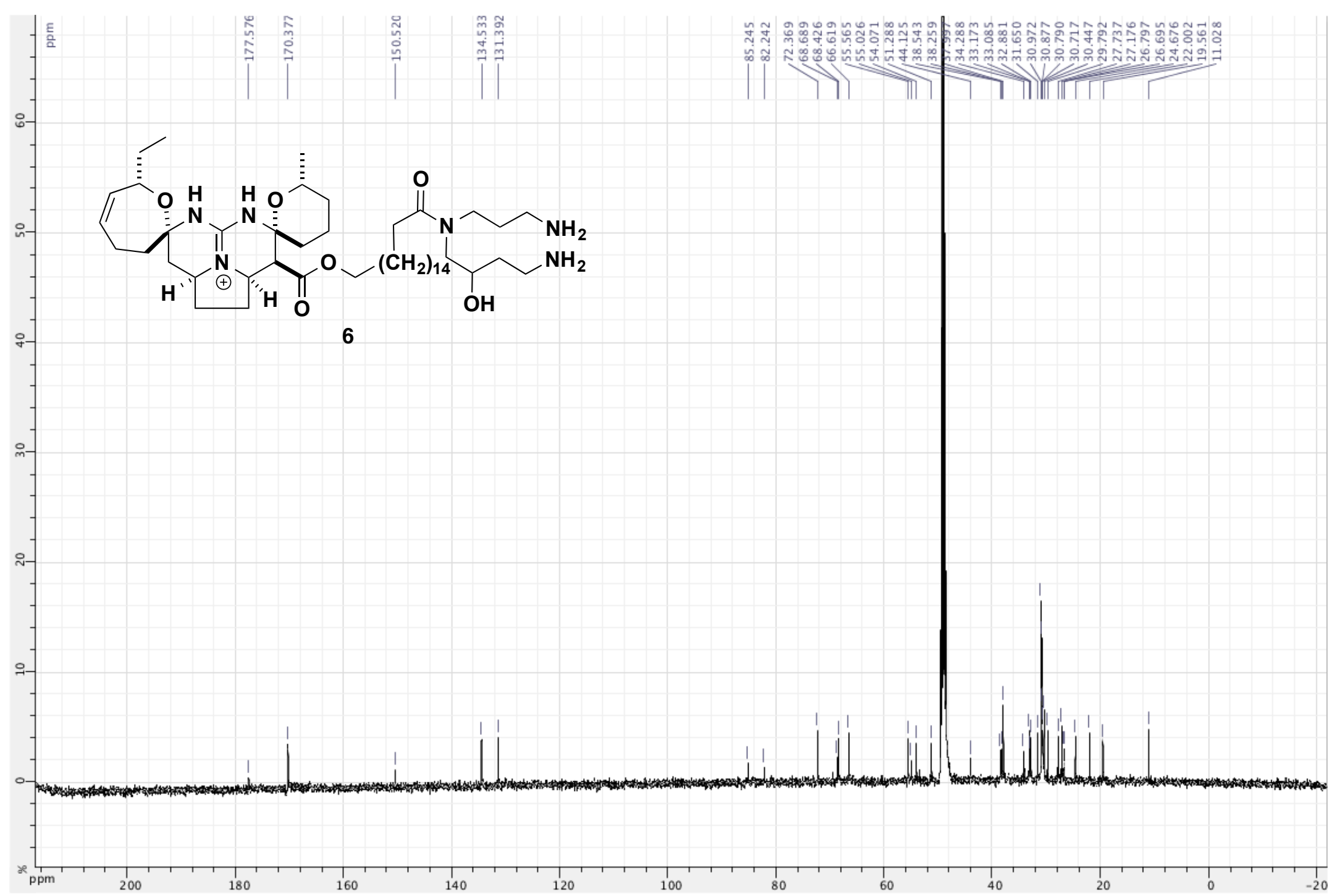

S34. ${ }^{1} \mathrm{H}-{ }^{1} \mathrm{H}$ COSY NMR spectrum of crambescidin 814 (6) in $\mathrm{CD}_{3} \mathrm{OD}(500 \mathrm{MHz})$. 


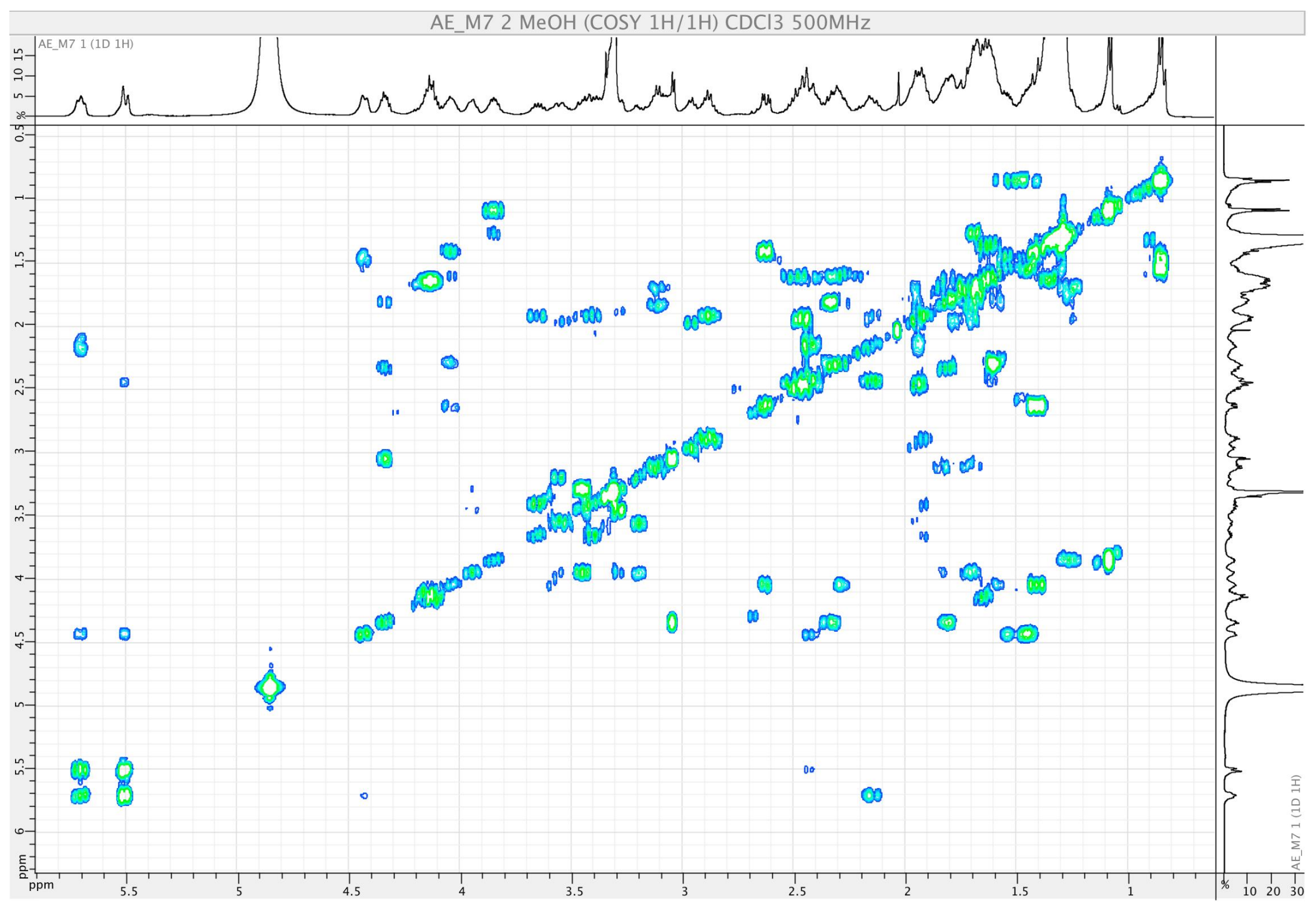

S35. HSQC NMR spectrum of crambescidin 814 (6) in $\mathrm{CD}_{3} \mathrm{OD}(500 \mathrm{MHz})$. 


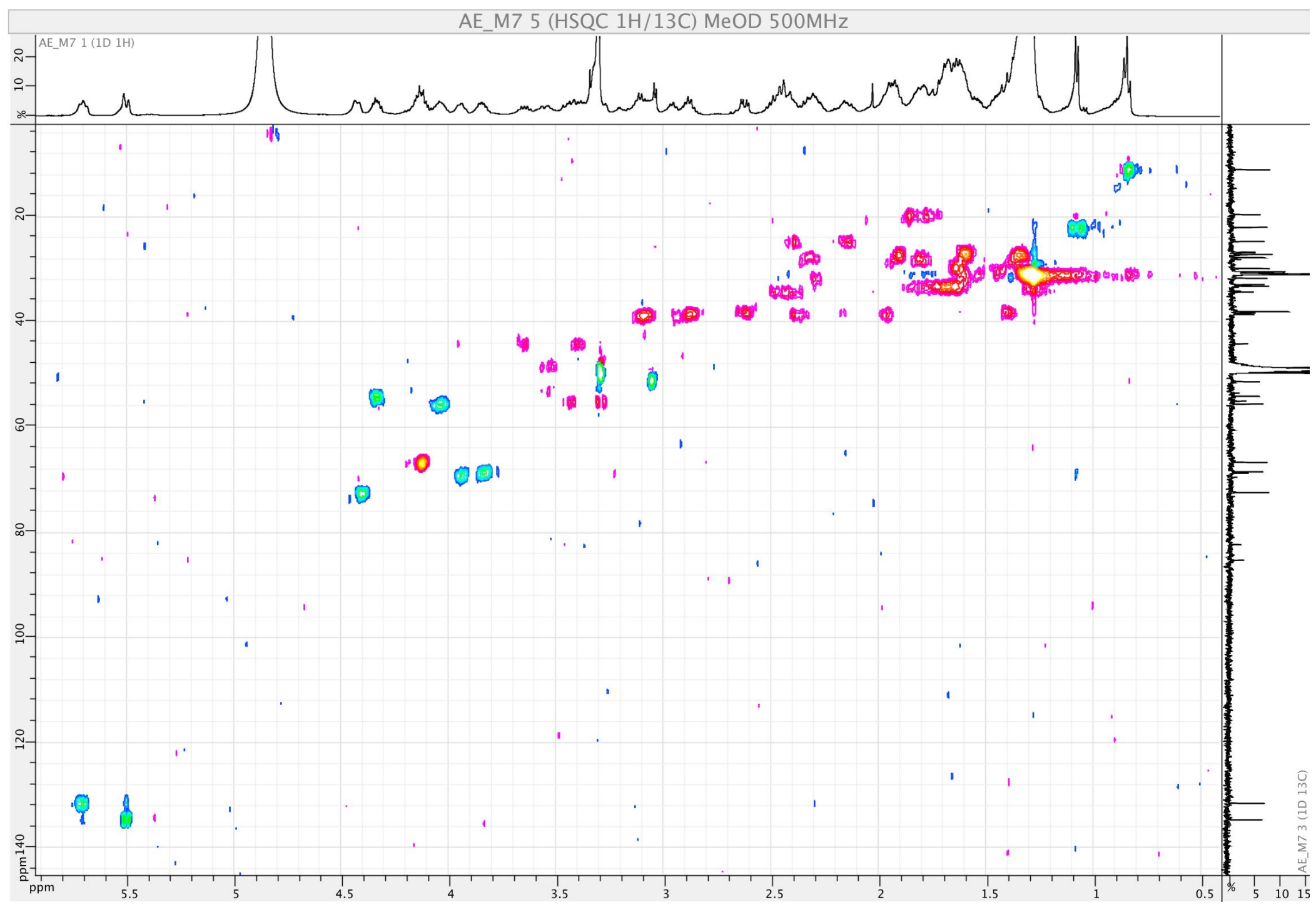

S36. ${ }^{1} \mathrm{H}_{-}{ }^{13} \mathrm{C}$ HMBC NMR spectrum of crambescidin 814 (6) in $\mathrm{CD}_{3} \mathrm{OD}(500 \mathrm{MHz})$. 


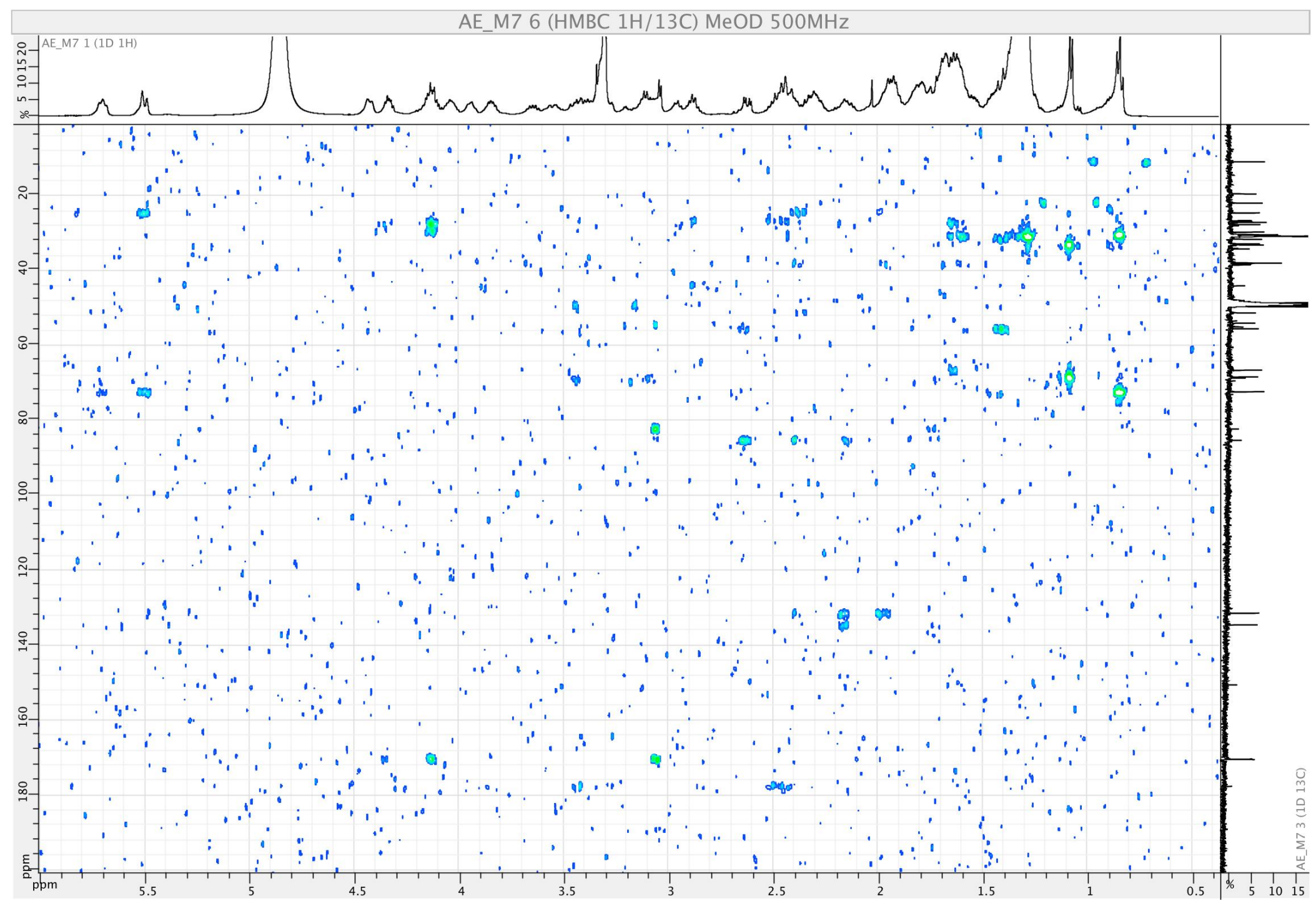

S37. UV spectrum of crambescidin 814 (6) in $\mathrm{MeOH}$. 


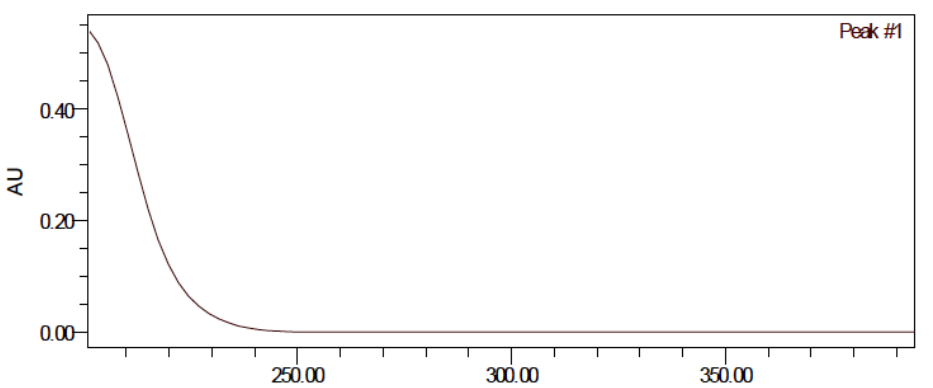

S38. IR spectrum of crambescidin 814 (6).

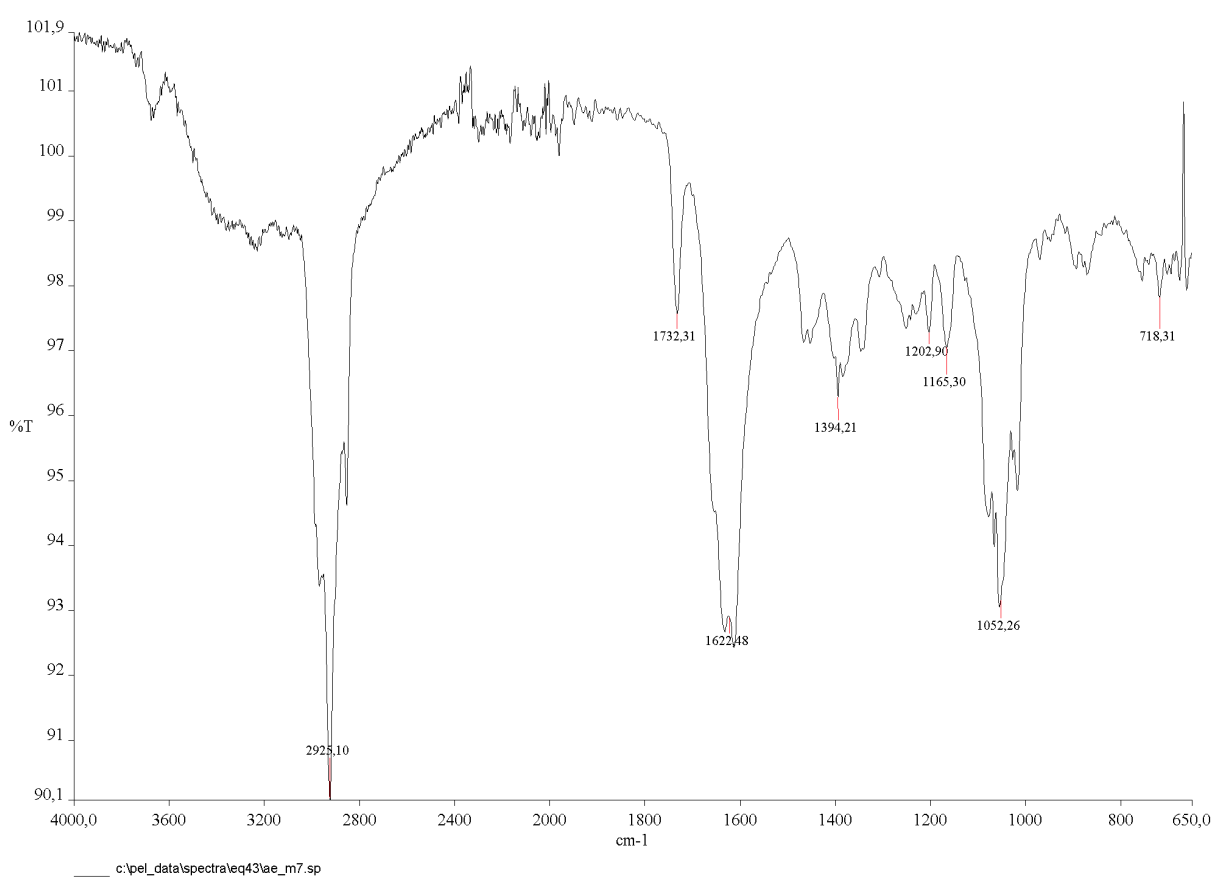

S39. ${ }^{1} \mathrm{H}$ NMR comparison of crambescidin 786 (5), crambescidin 800 (12) and crambescidin 814 (6) in $\mathrm{CD}_{3} \mathrm{OD}(500 \mathrm{MHz})$. 


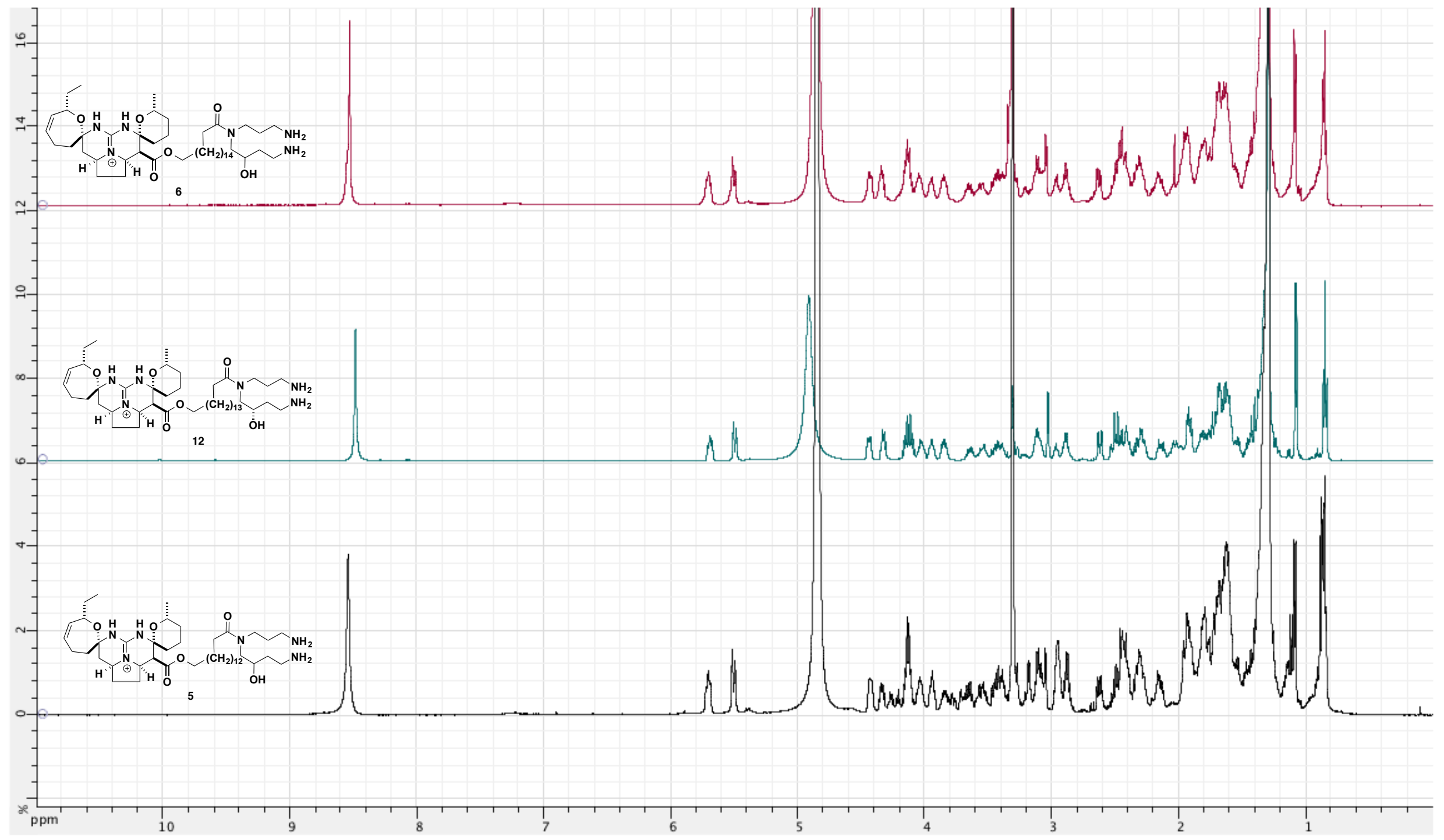

S40. ${ }^{1} \mathrm{H}$ NMR spectrum of norcrambescidic acid (7) in $\mathrm{CD}_{3} \mathrm{OD}(600 \mathrm{MHz})$. 


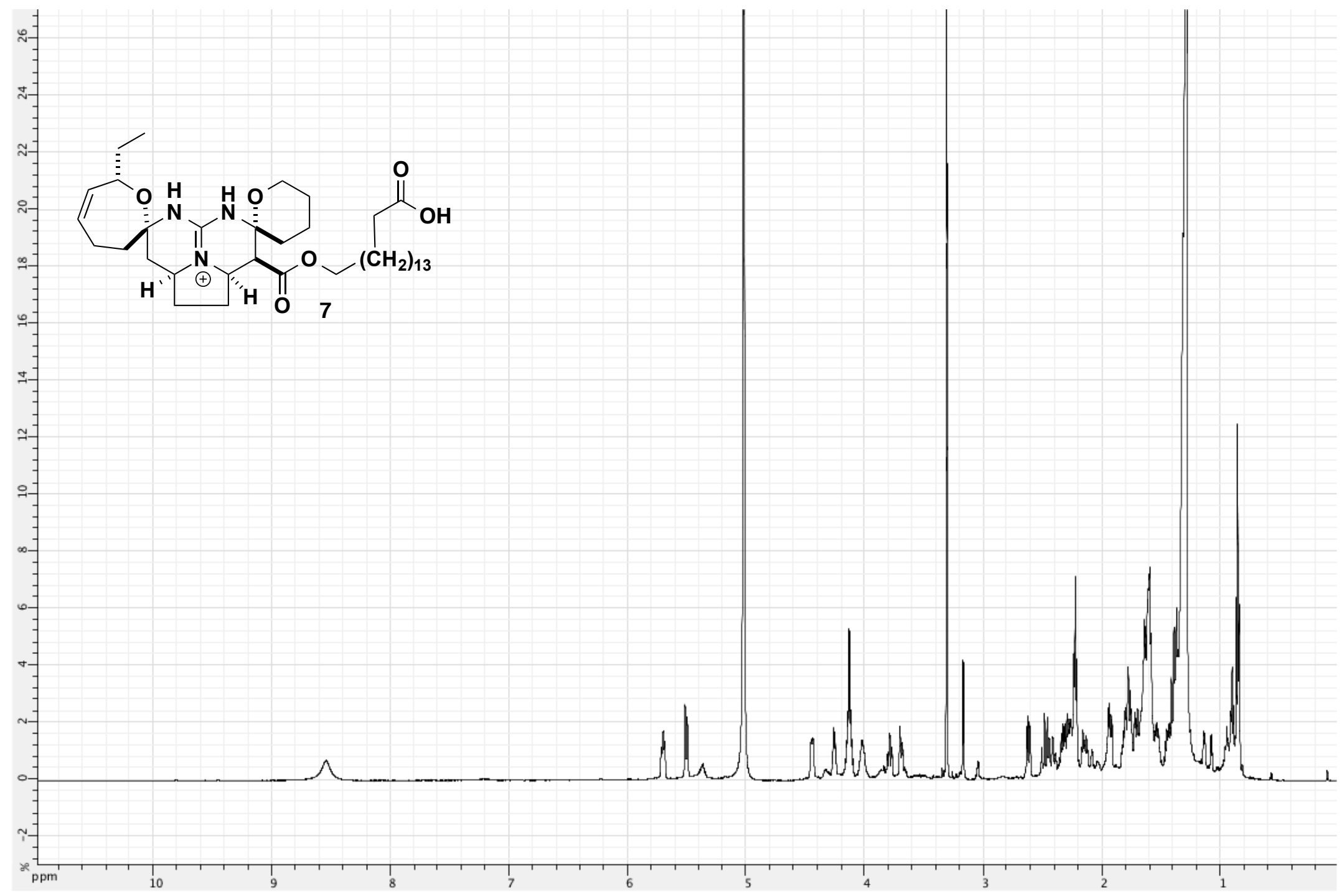

S41. ${ }^{13} \mathrm{C}$ NMR spectrum of norcrambescidic acid (7) in $\mathrm{CD}_{3} \mathrm{OD}(125 \mathrm{MHz})$. 


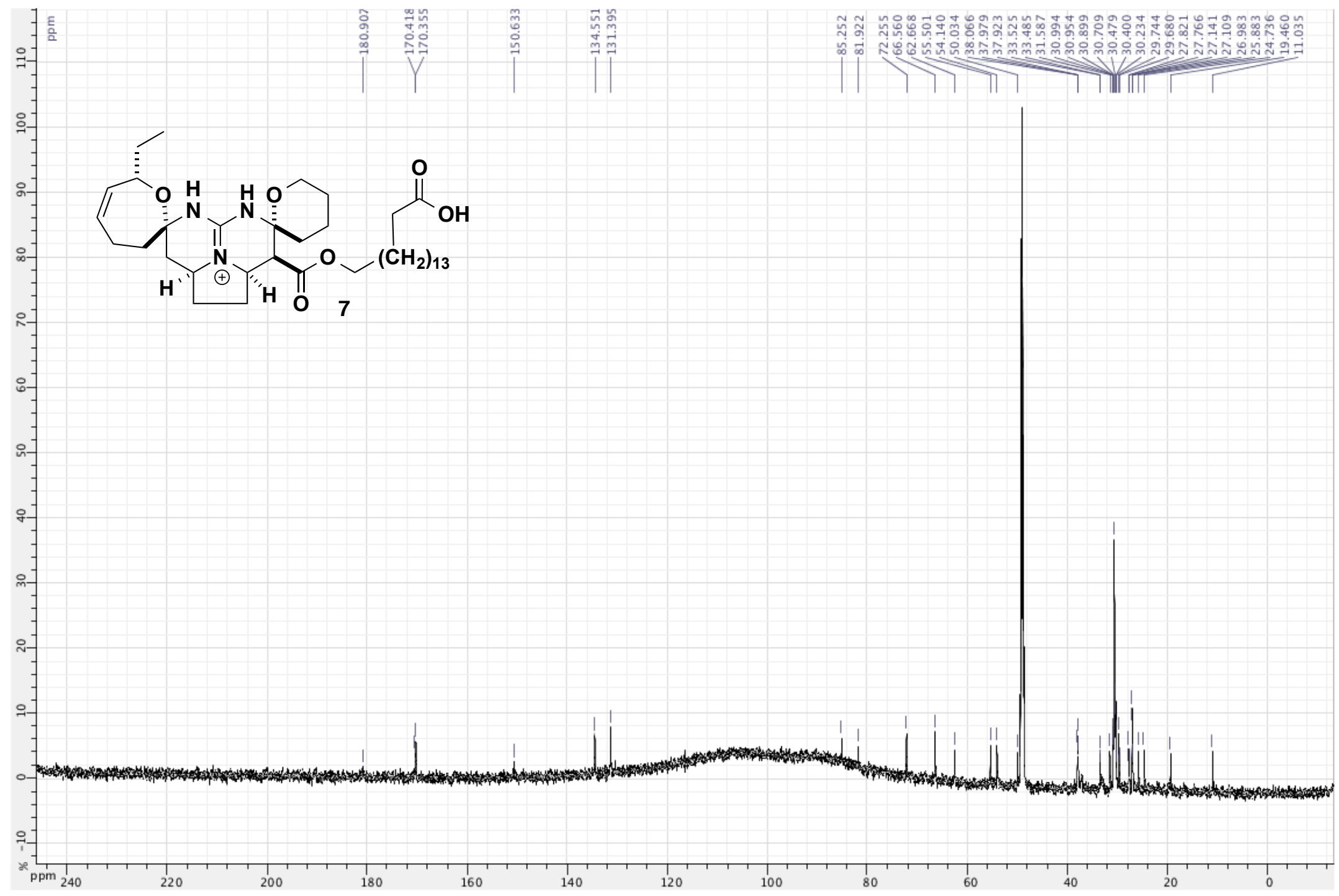

S42. ${ }^{1} \mathrm{H}-{ }^{1} \mathrm{H}$ COSY NMR spectrum of norcrambescidic acid (7) in $\mathrm{CD}_{3} \mathrm{OD}(600 \mathrm{MHz})$. 


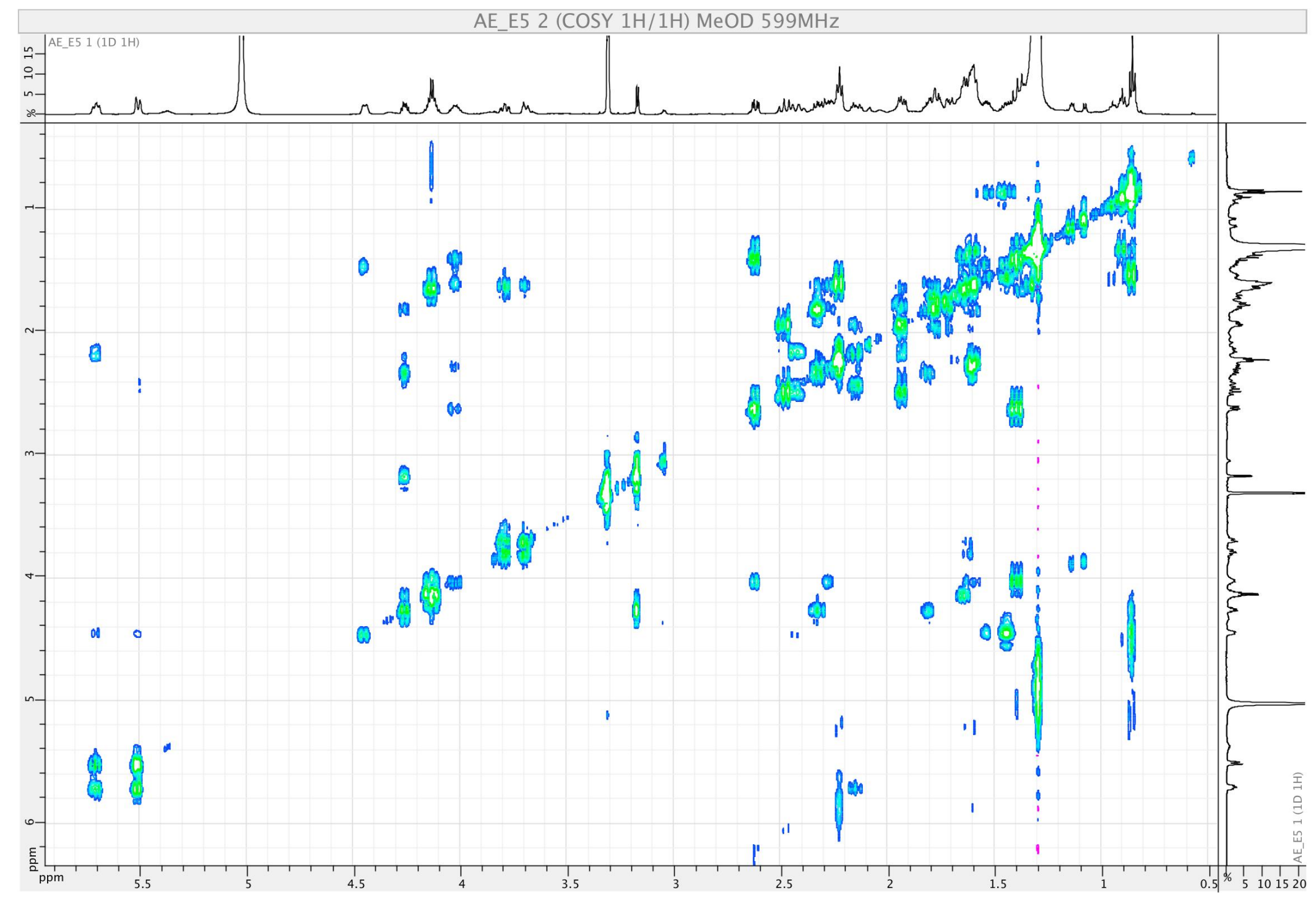

S43. HSQC NMR spectrum of norcrambescidic acid (7) in $\mathrm{CD}_{3} \mathrm{OD}(600 \mathrm{MHz})$. 


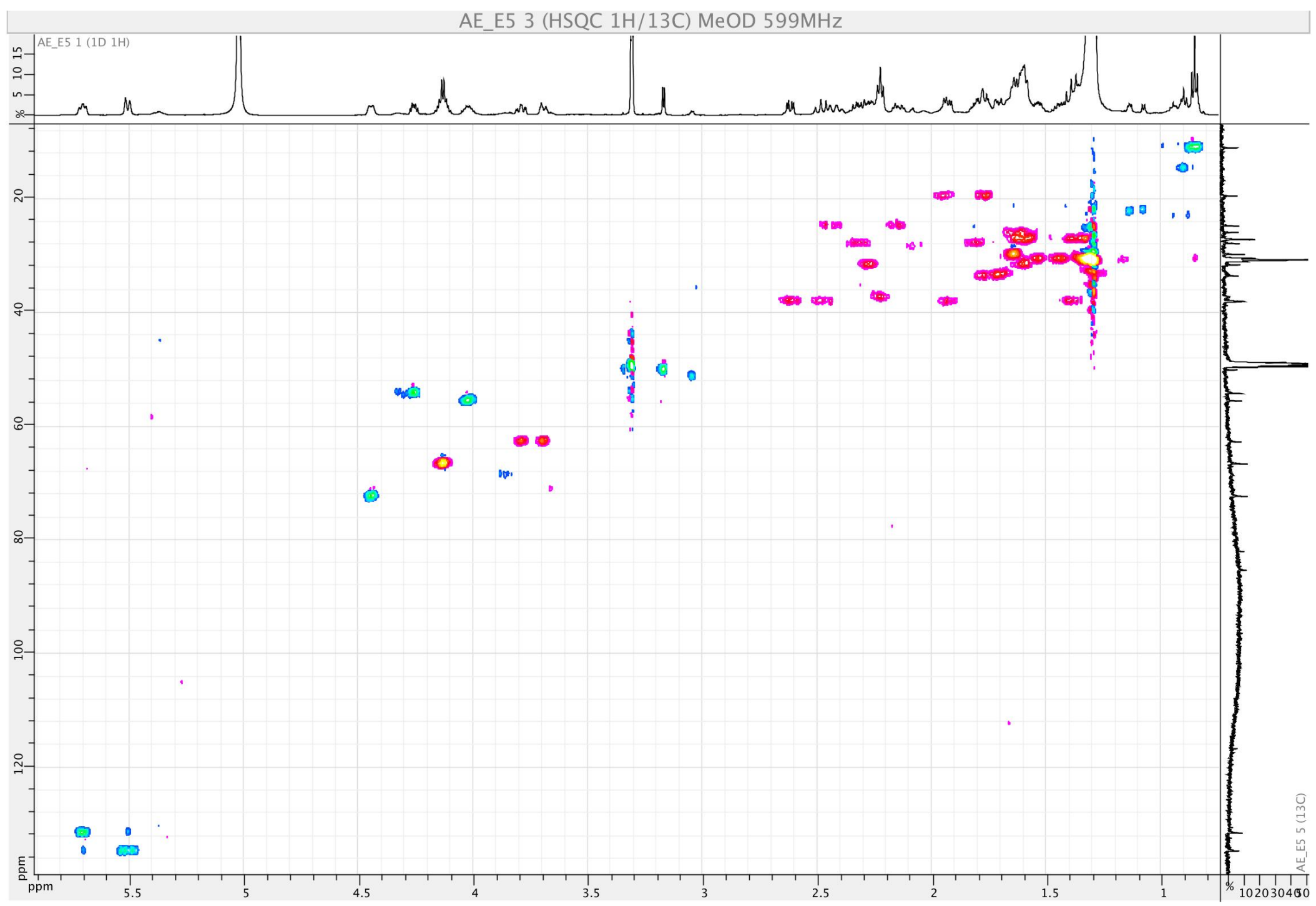

S44. ${ }^{1} \mathrm{H}_{-}{ }^{13} \mathrm{C} \mathrm{HMBC}$ NMR spectrum of norcrambescidic acid (7) in $\mathrm{CD}_{3} \mathrm{OD}(600 \mathrm{MHz})$. 


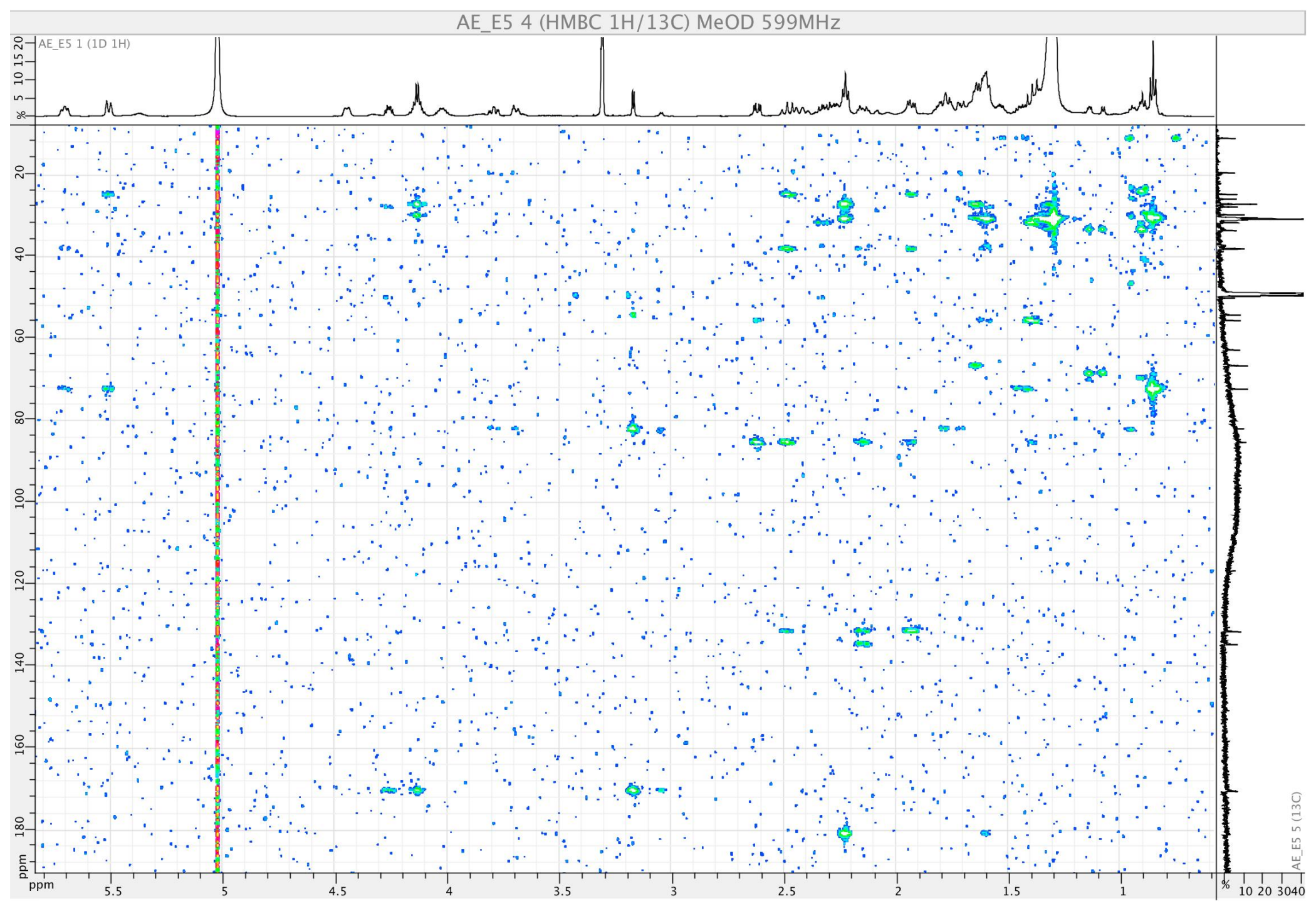

S45. UV spectrum of norcrambescidic acid (7) in $\mathrm{MeOH}$. 


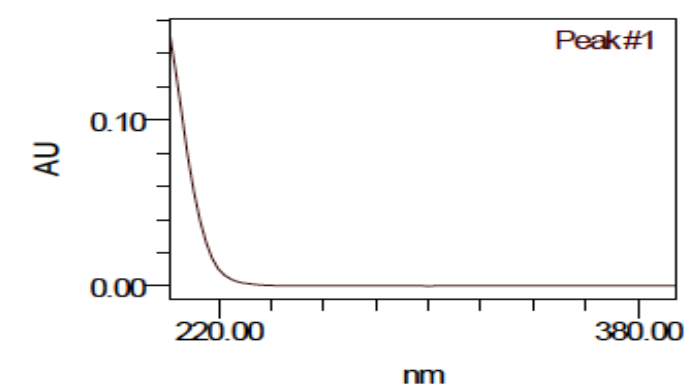

S46. IR spectrum of norcrambescidic acid (7).

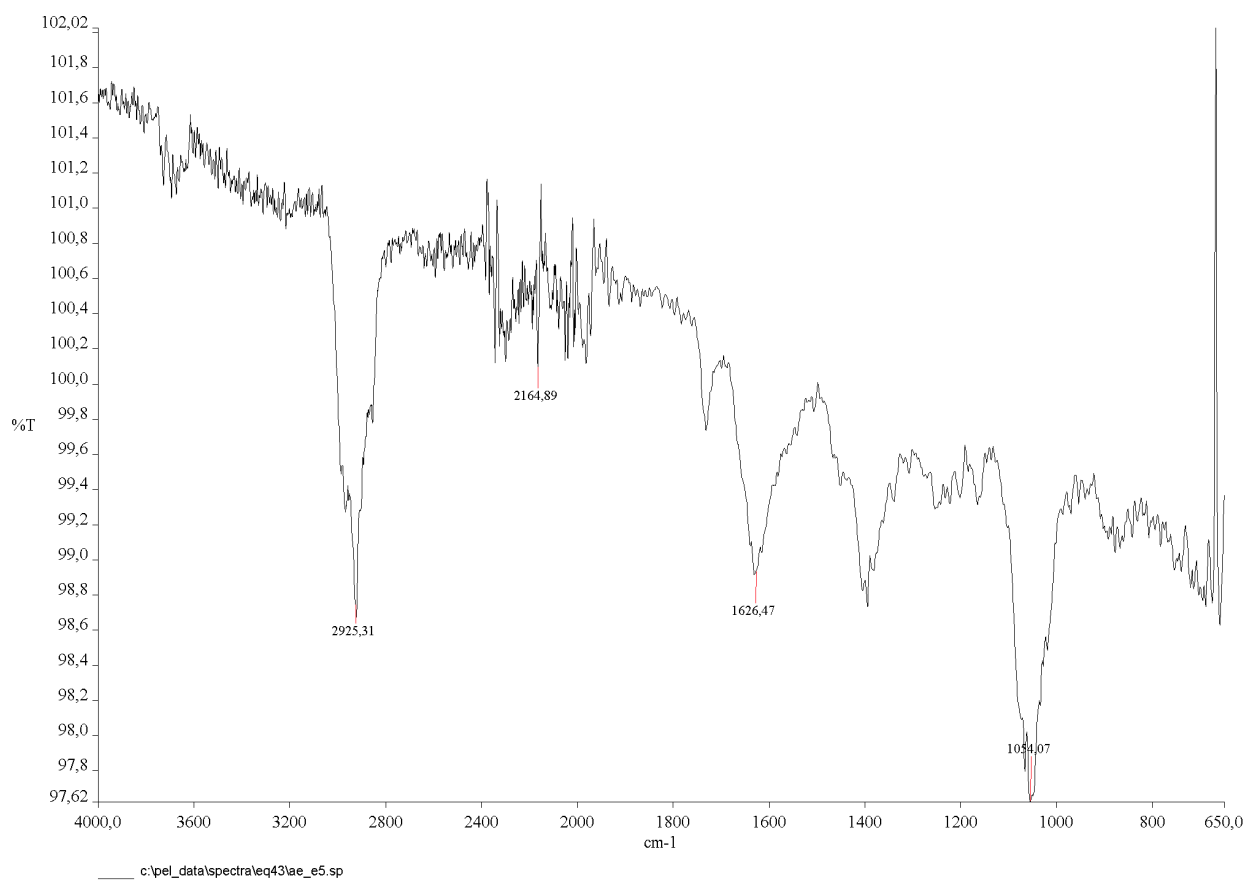

S47. ${ }^{1} \mathrm{H}$ NMR spectrum of monalidine A (8) in $\mathrm{CD}_{3} \mathrm{OD}(500 \mathrm{MHz})$. 


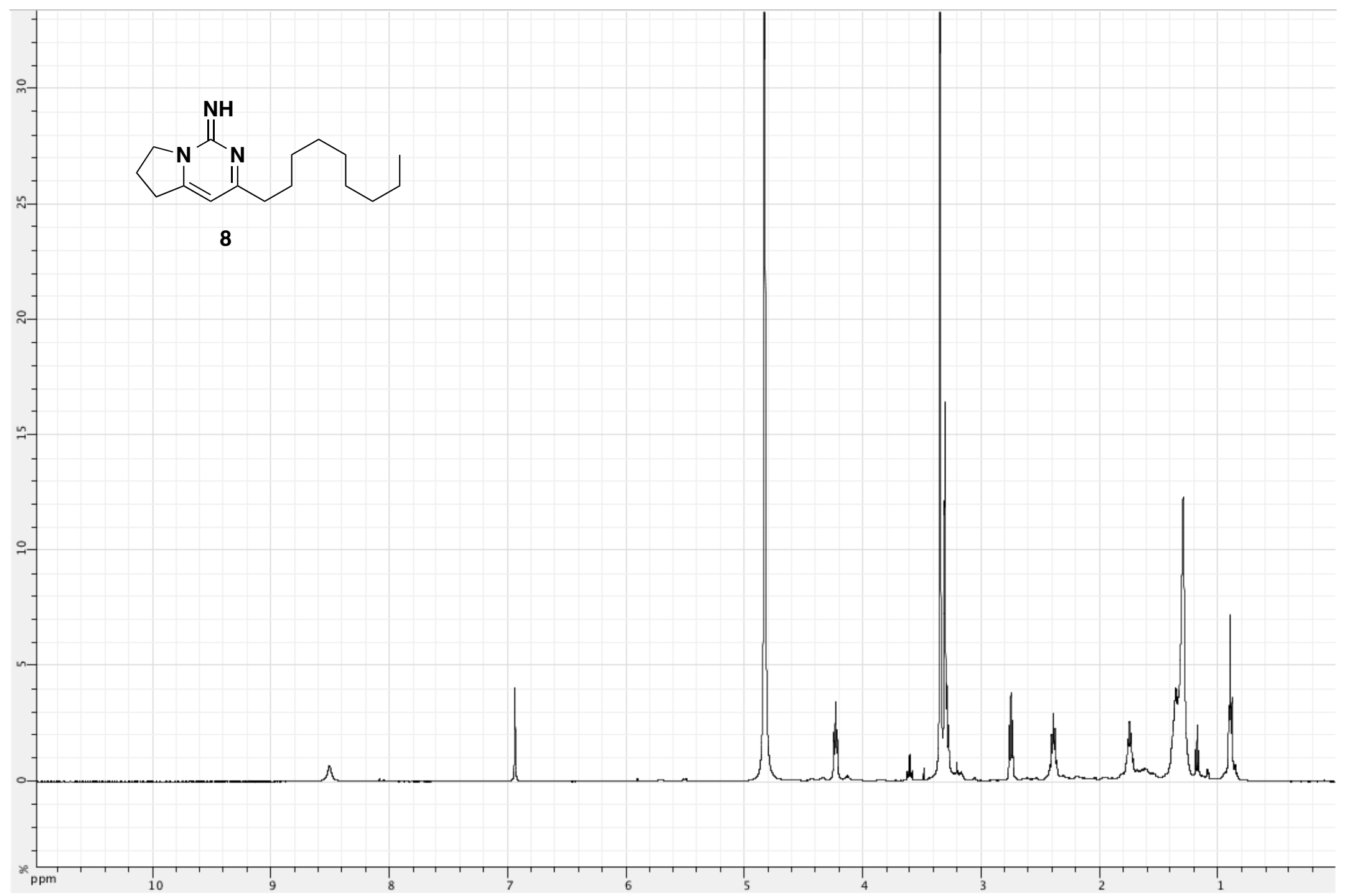

S48. ${ }^{13} \mathrm{C}$ NMR spectrum of monalidine $\mathrm{A}(\mathbf{8})$ in $\mathrm{CD}_{3} \mathrm{OD}(125 \mathrm{MHz})$. 


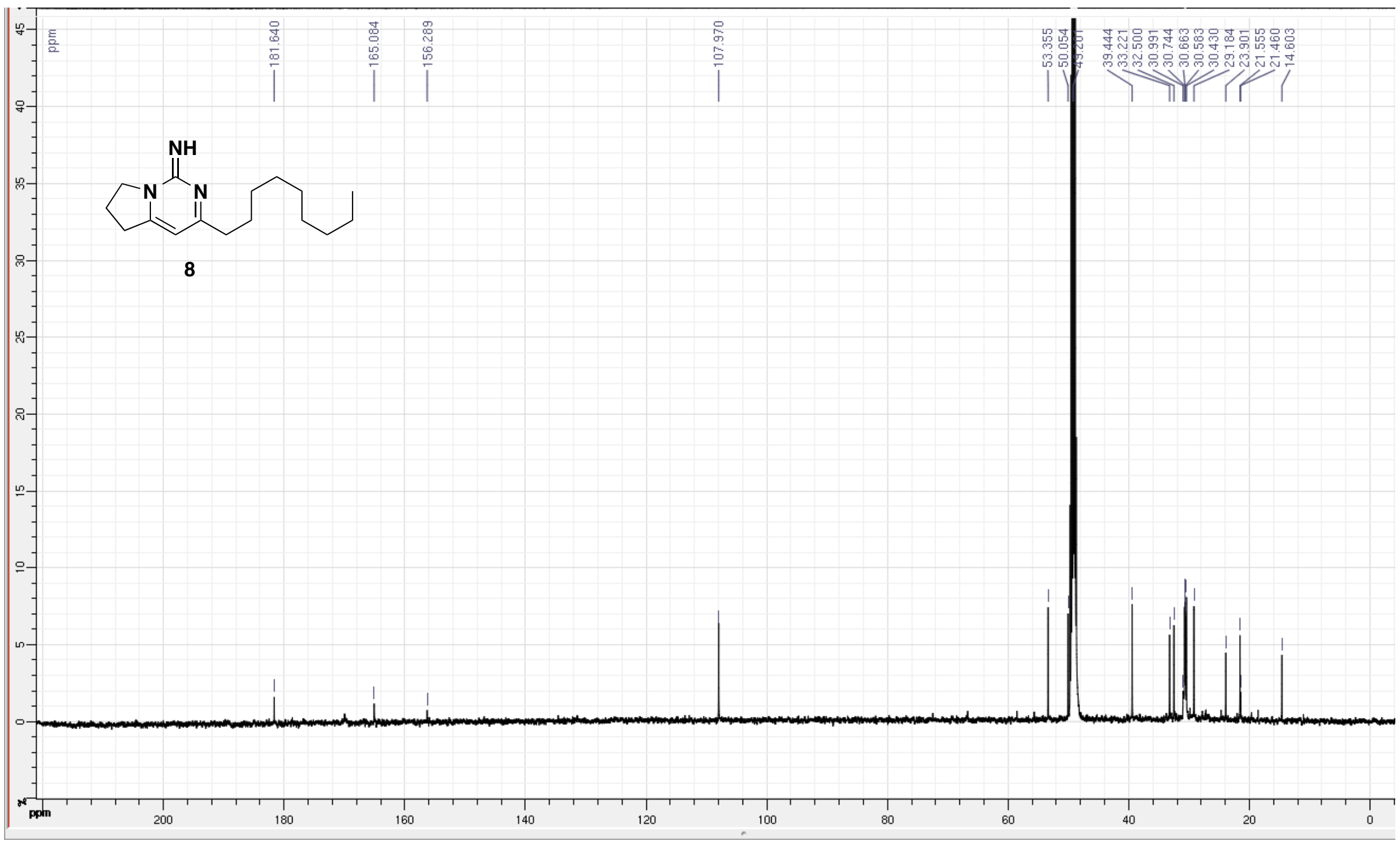

S49. ${ }^{1} \mathrm{H}$ NMR spectrum of (-)-crambescin A2 392 (9) in $\mathrm{CD}_{3} \mathrm{OD}(500 \mathrm{MHz})$ 


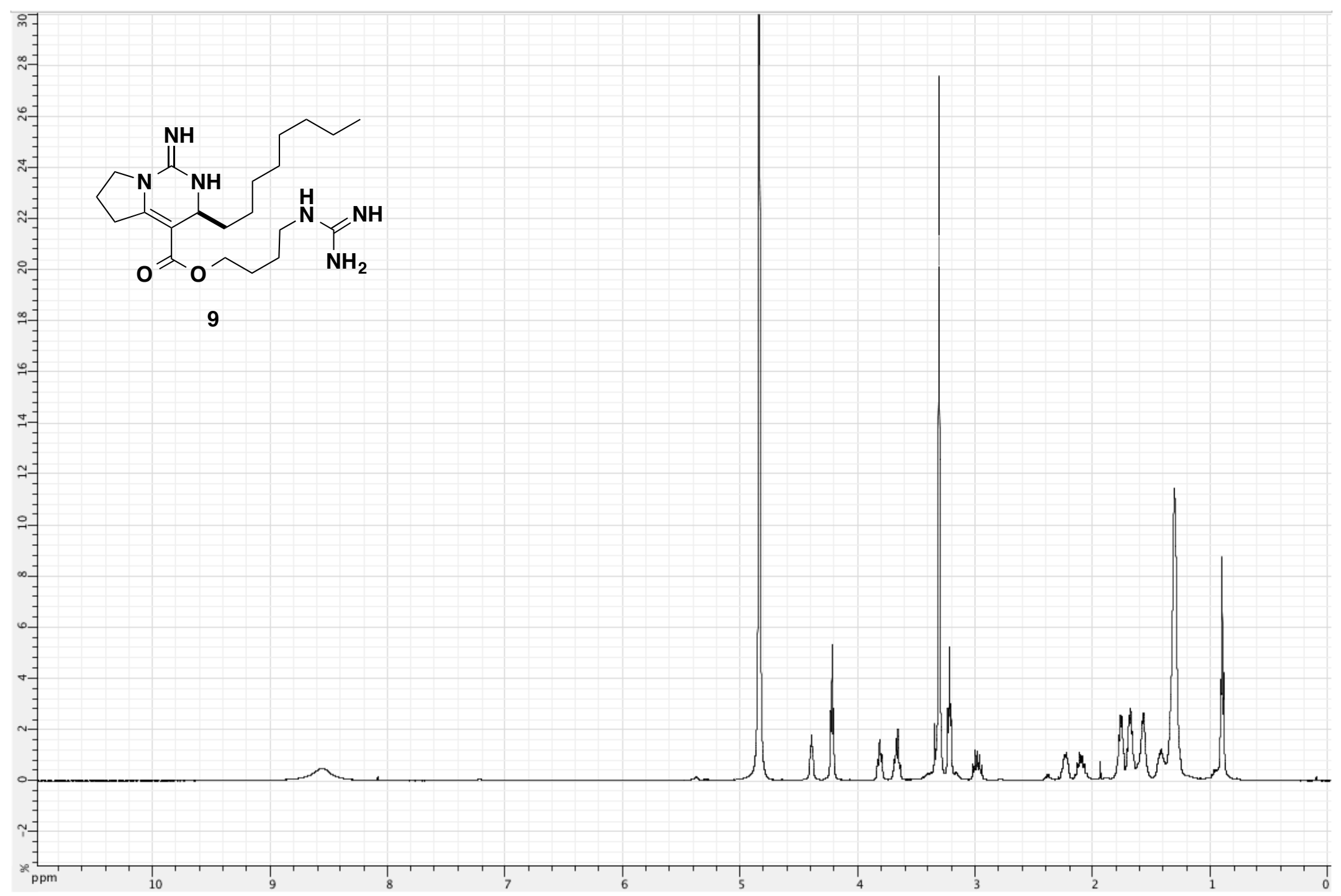

S50. ${ }^{13} \mathrm{C}$ NMR spectrum of (-)-crambescin $\mathrm{A} 2392$ (9) in $\mathrm{CD}_{3} \mathrm{OD}(125 \mathrm{MHz})$. 


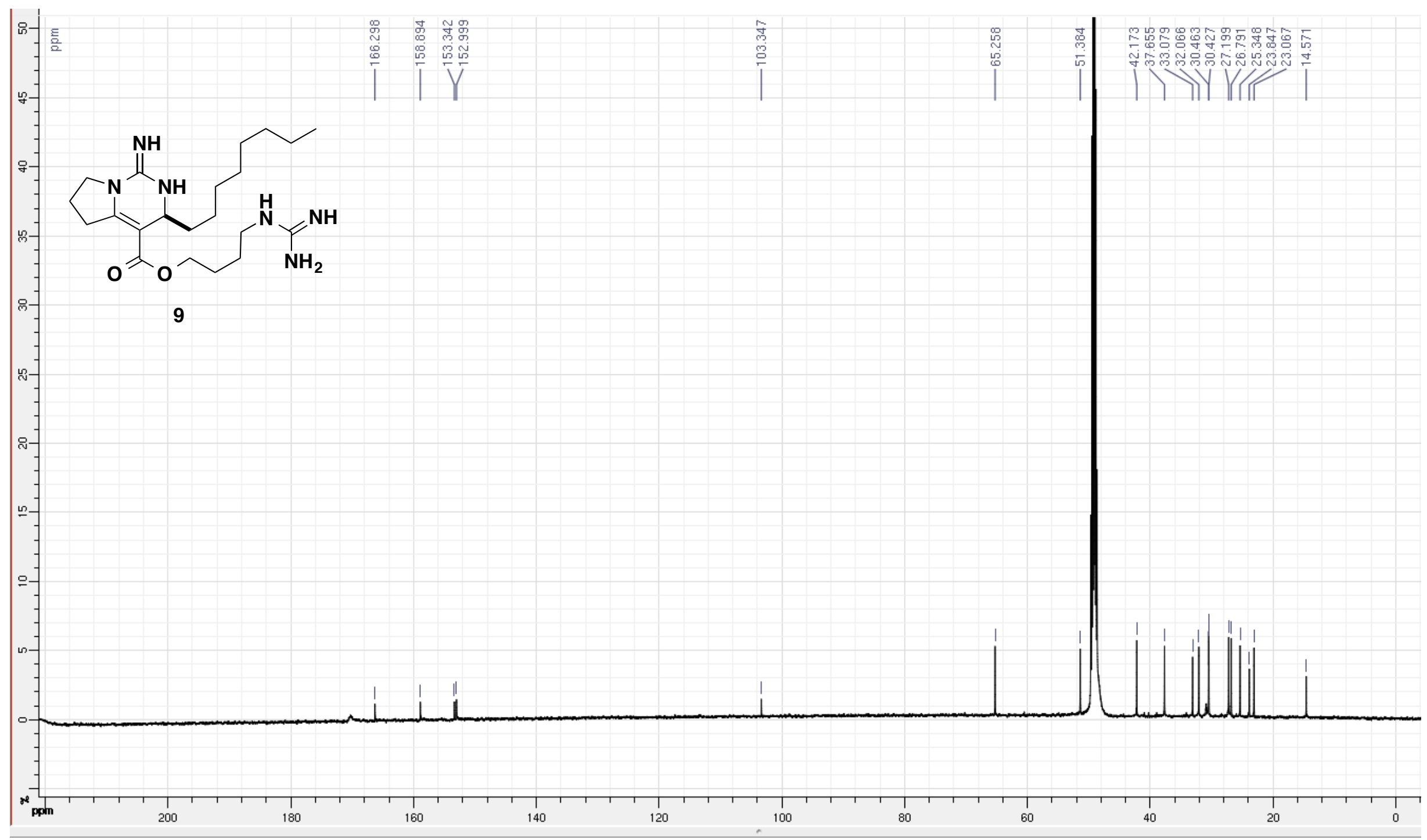

S51. ${ }^{1} \mathrm{H}$ NMR spectrum of (-)-crambescin A2 406 (10) in $\mathrm{CD}_{3} \mathrm{OD}(500 \mathrm{MHz})$. 


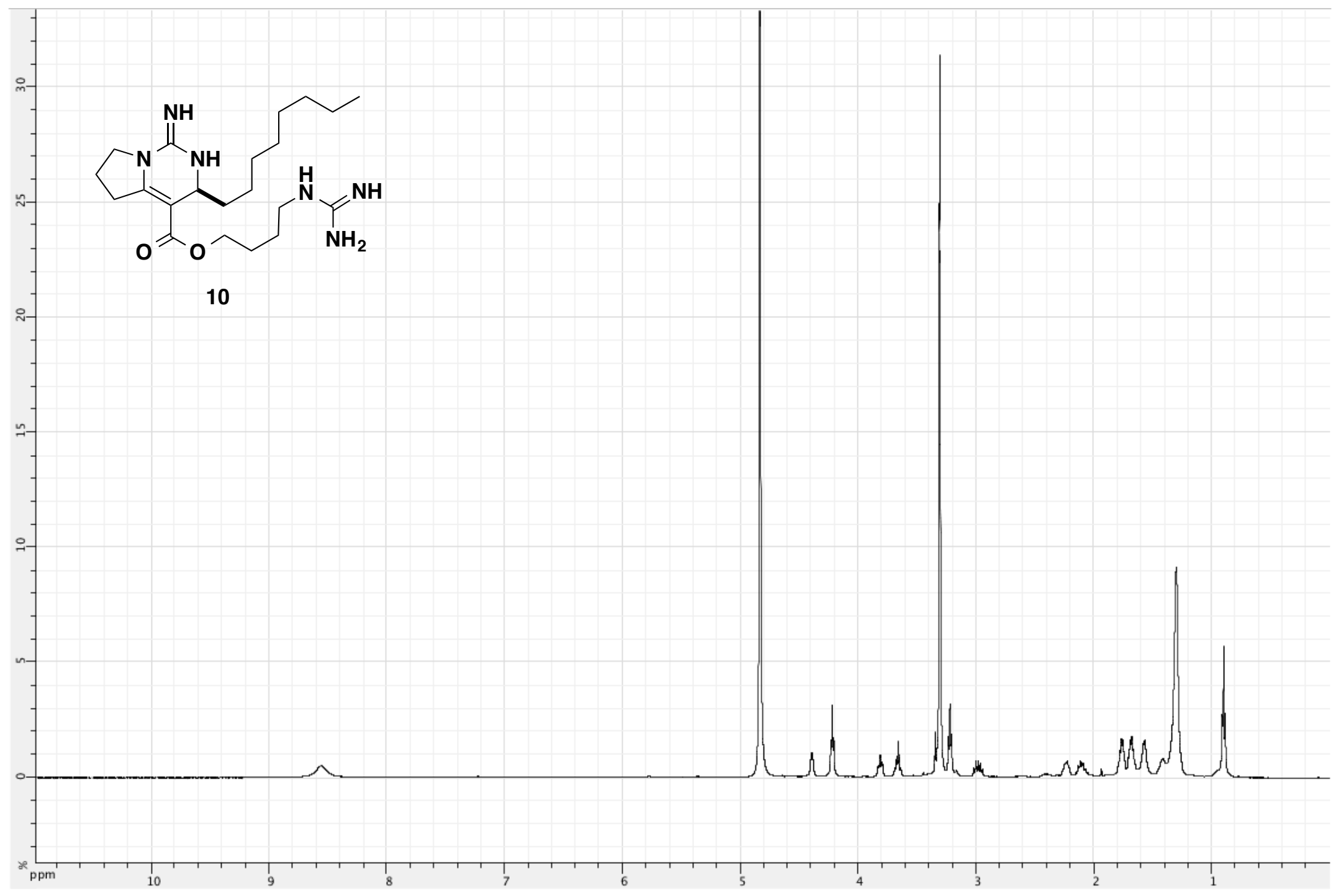

S52. ${ }^{13} \mathrm{C}$ NMR spectrum of (-)-crambescin $\mathrm{A} 2406(\mathbf{1 0})$ in $\mathrm{CD}_{3} \mathrm{OD}(125 \mathrm{MHz})$. 


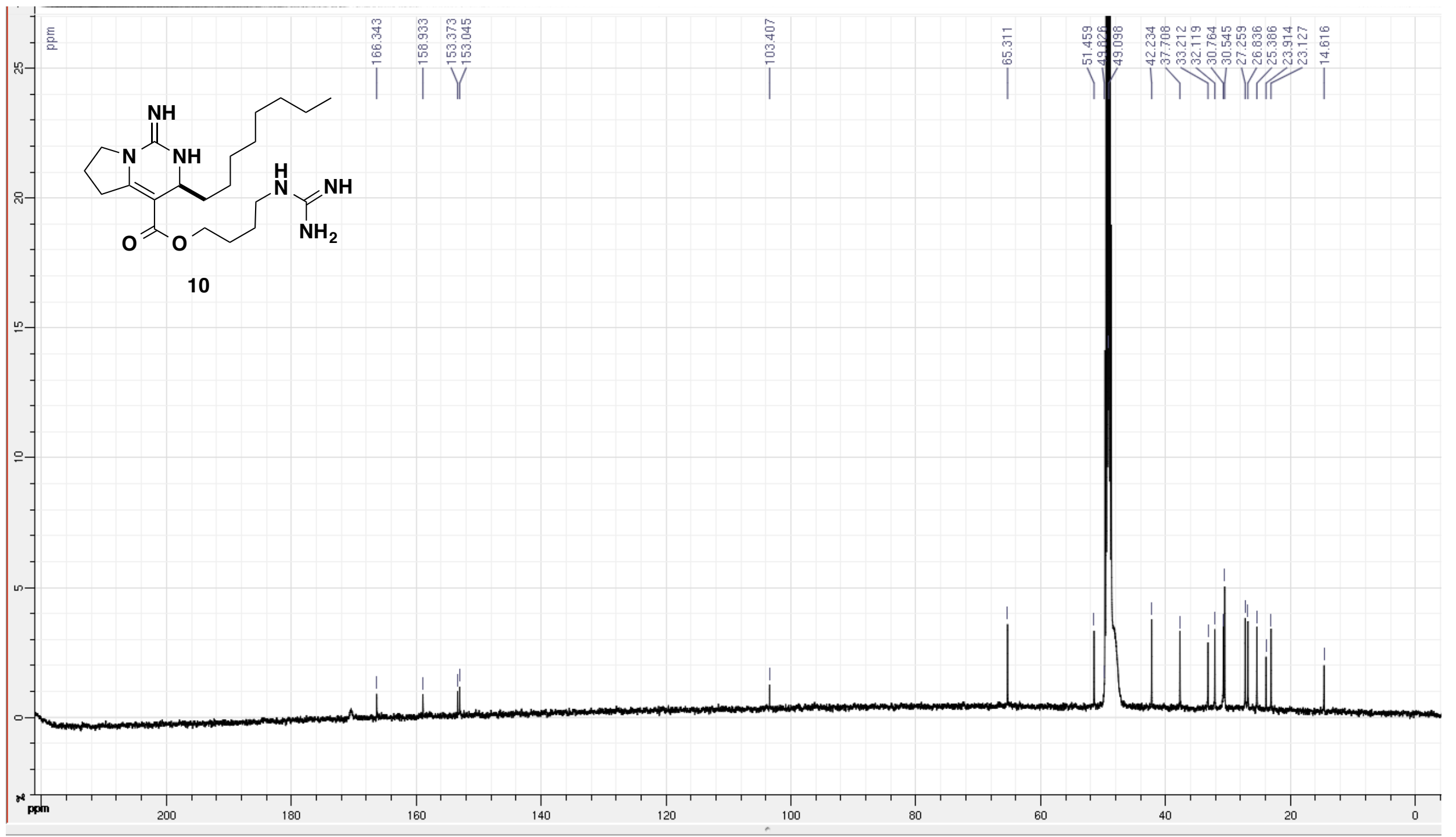

S53. ${ }^{1} \mathrm{H}$ NMR spectrum of (-)-crambescin A2 420 (11) in $\mathrm{CD}_{3} \mathrm{OD}(500 \mathrm{MHz})$. 


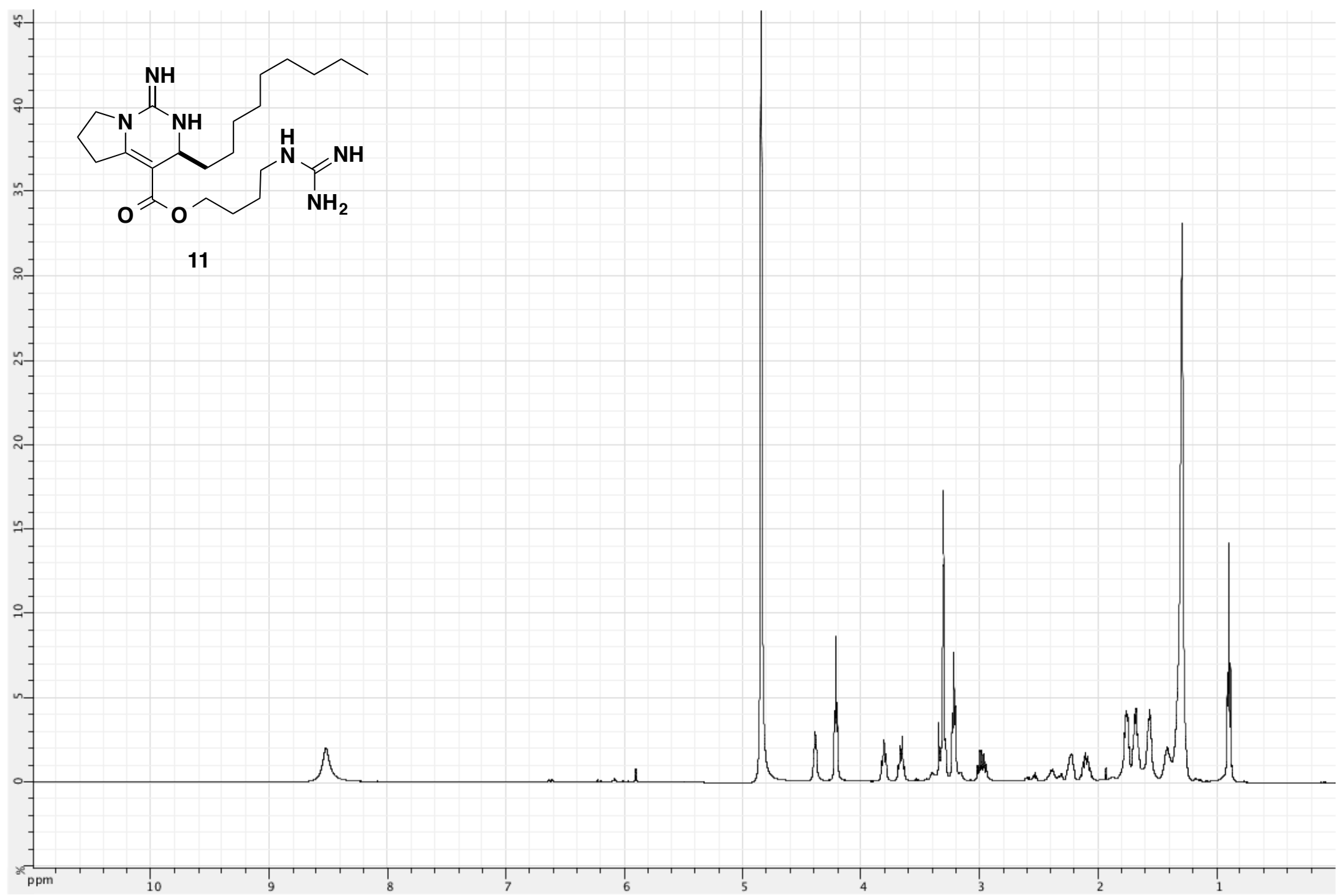

554. ${ }^{13} \mathrm{C}$ NMR spectrum of (-)-crambescin A2 420 (11) in $\mathrm{CD}_{3} \mathrm{OD}(125 \mathrm{MHz})$. 


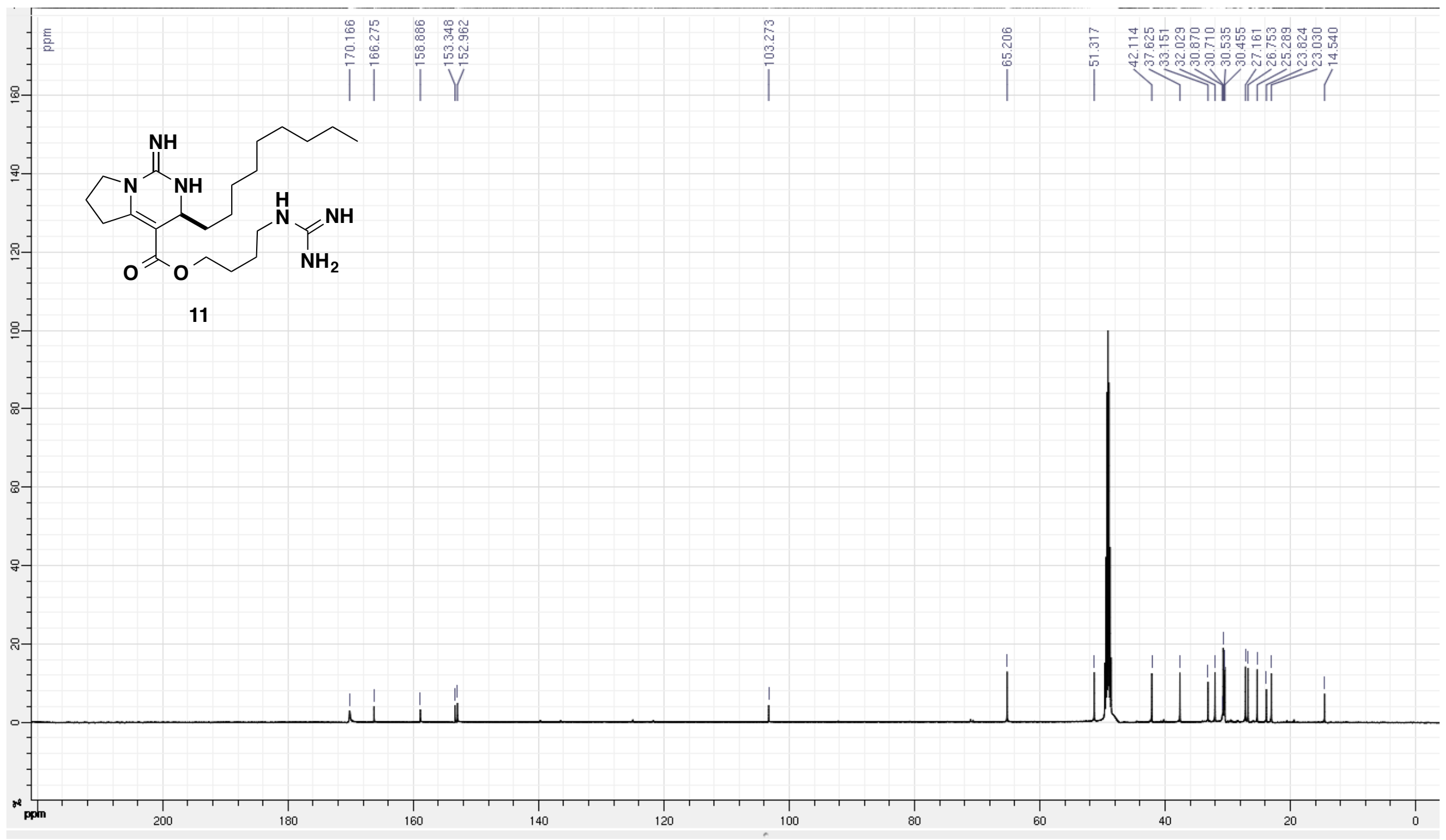

S55. ${ }^{1} \mathrm{H}$ NMR comparision for (-)-crambescins A2 392 (9), 406 (10) and 420 (11) in $\mathrm{CD}_{3} \mathrm{OD}(500 \mathrm{MHz})$. 


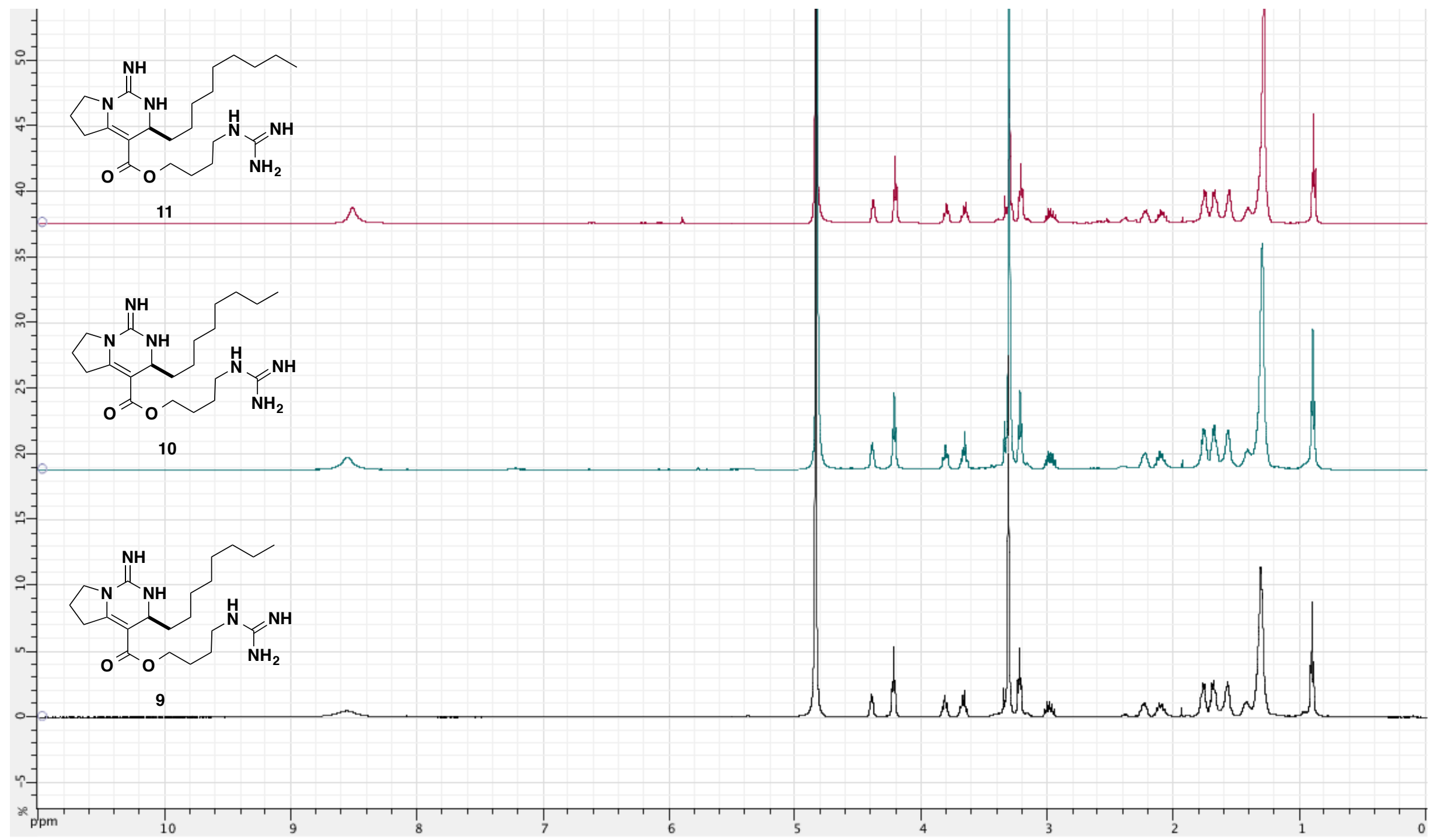

S56. ECD spectra for (-)-crambescins A2 392 (10), 406 (11) and 420 (12) in form of TFA salt. 


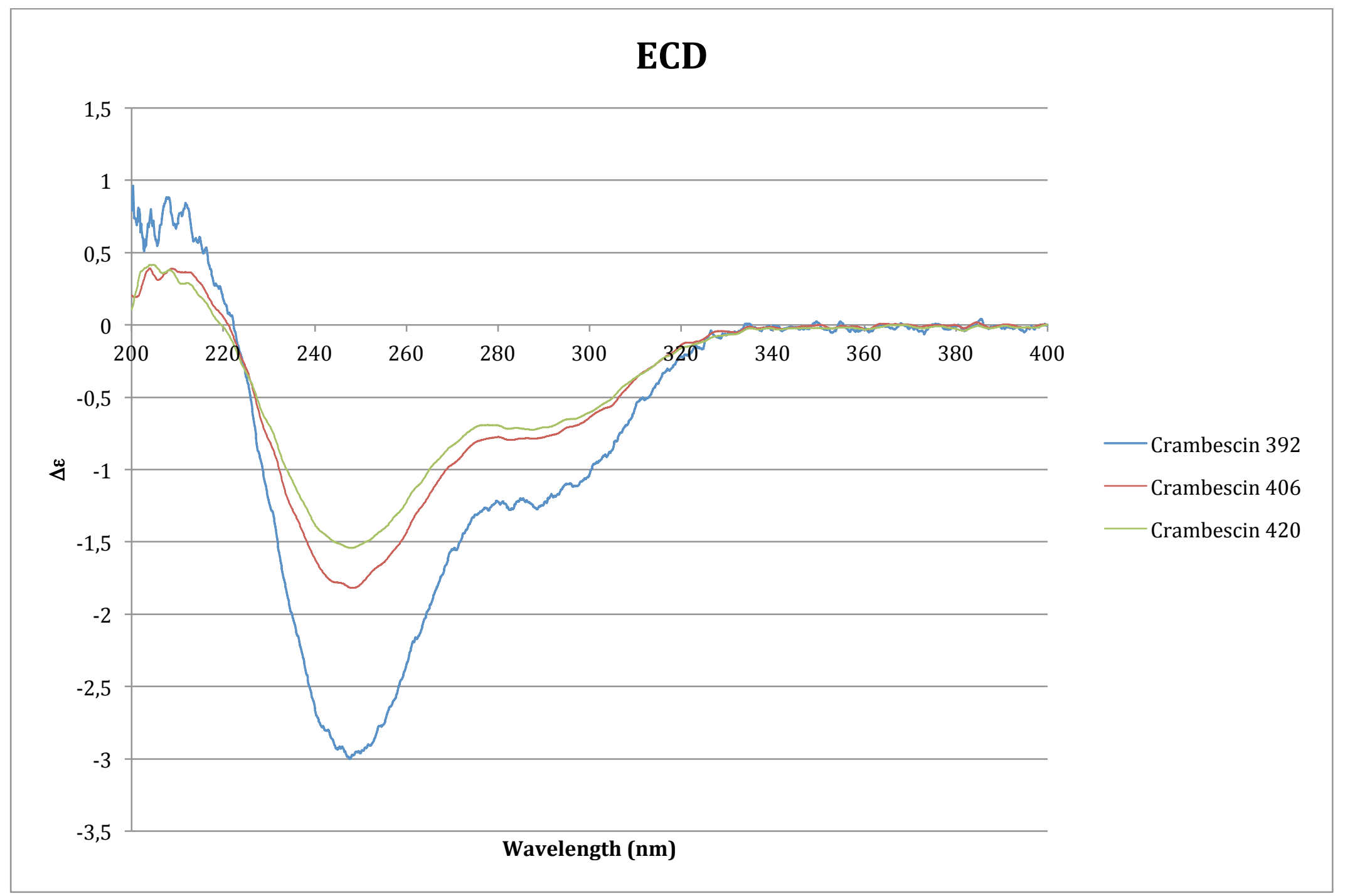

S57. ${ }^{1} \mathrm{H}$ NMR spectrum of crambescidin $800(\mathbf{1 2})$ in $\mathrm{CD}_{3} \mathrm{OD}(500 \mathrm{MHz})$. 


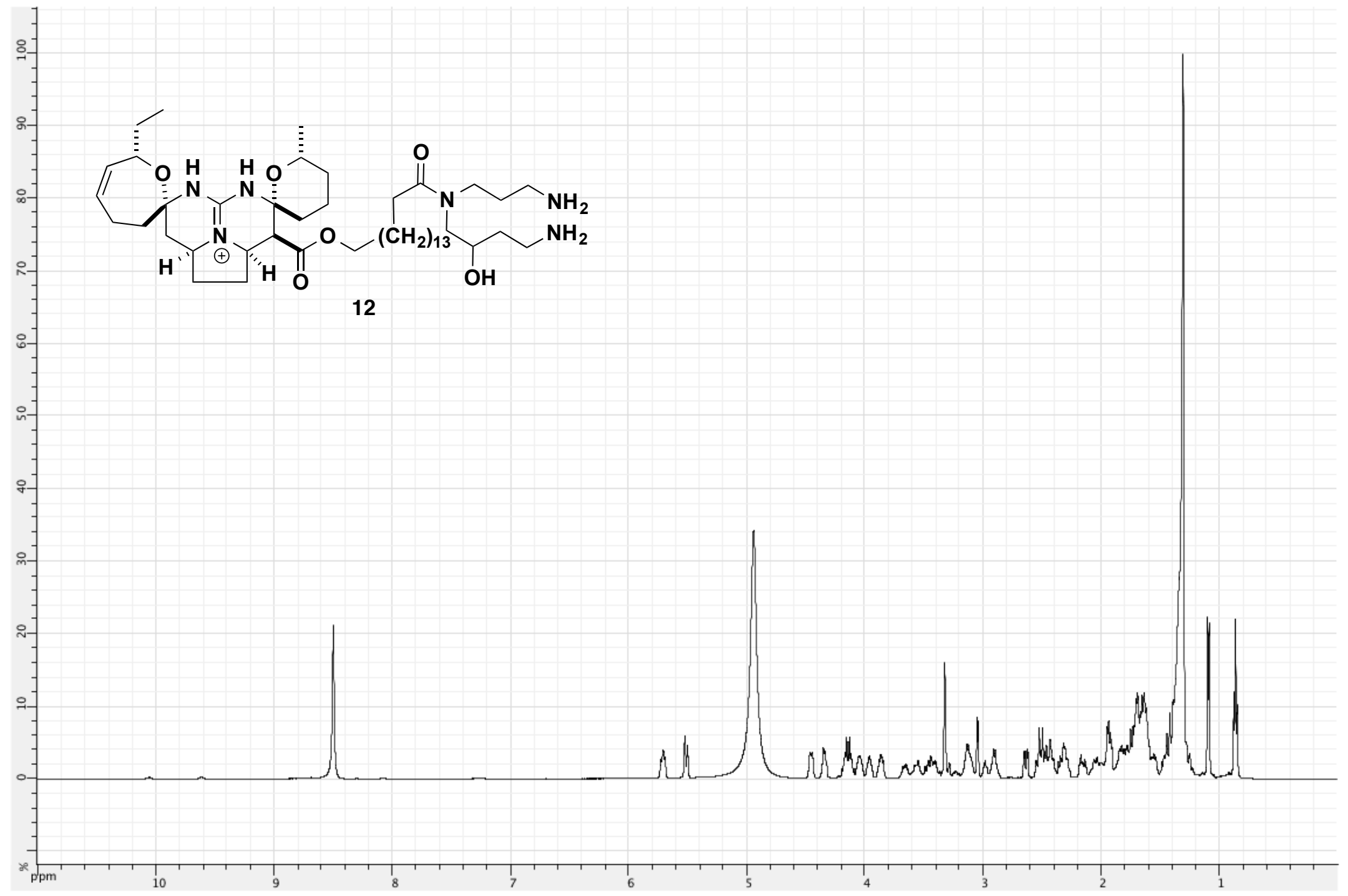

S58. ${ }^{13} \mathrm{C}$ NMR spectrum of crambescidin 800 (12) in $\mathrm{CD}_{3} \mathrm{OD}(125 \mathrm{MHz})$. 


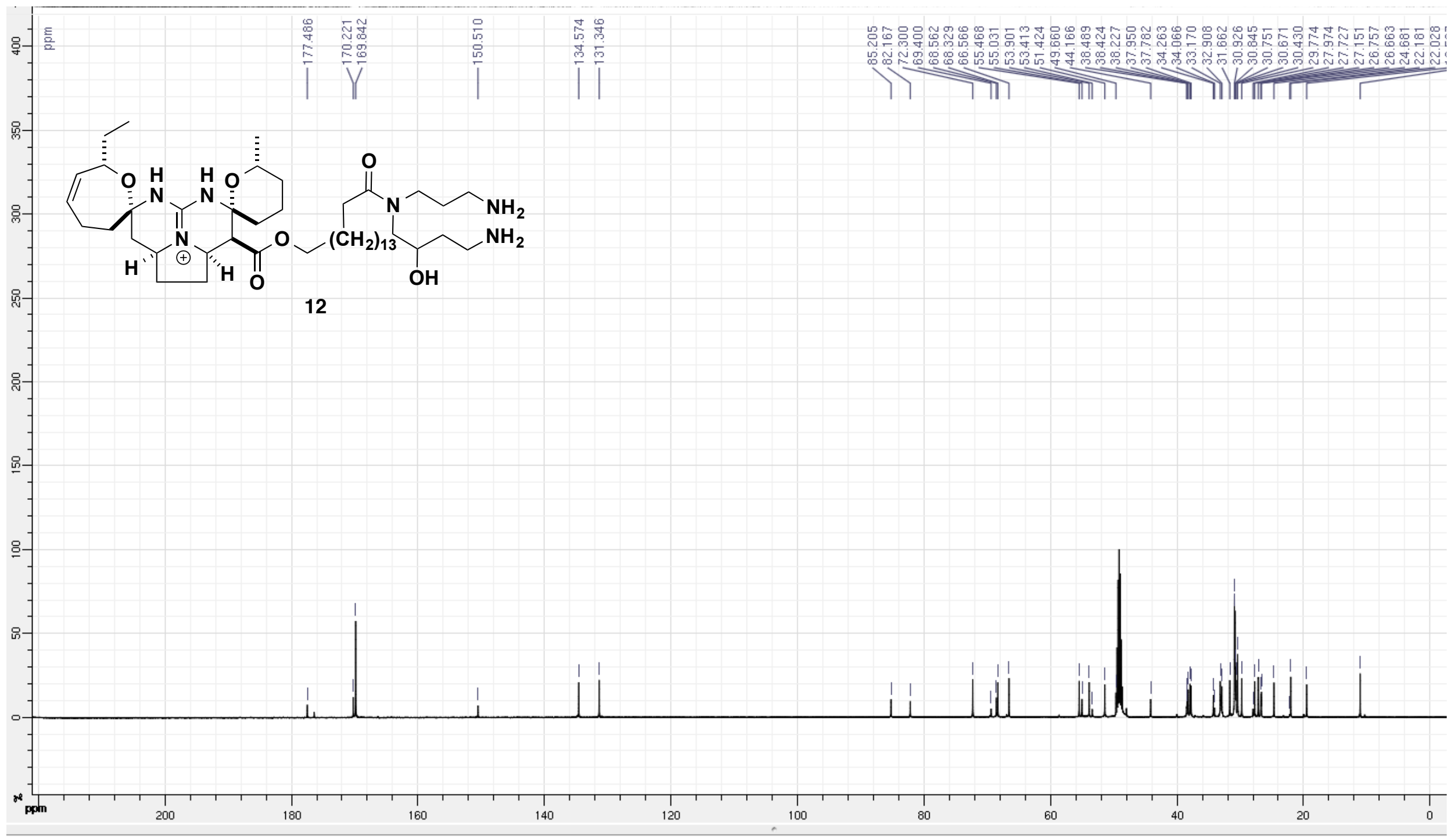

S59. ${ }^{1} \mathrm{H}$ NMR spectrum of crambescidin $826(\mathbf{1 3})$ in $\mathrm{CD}_{3} \mathrm{OD}(500 \mathrm{MHz})$. 


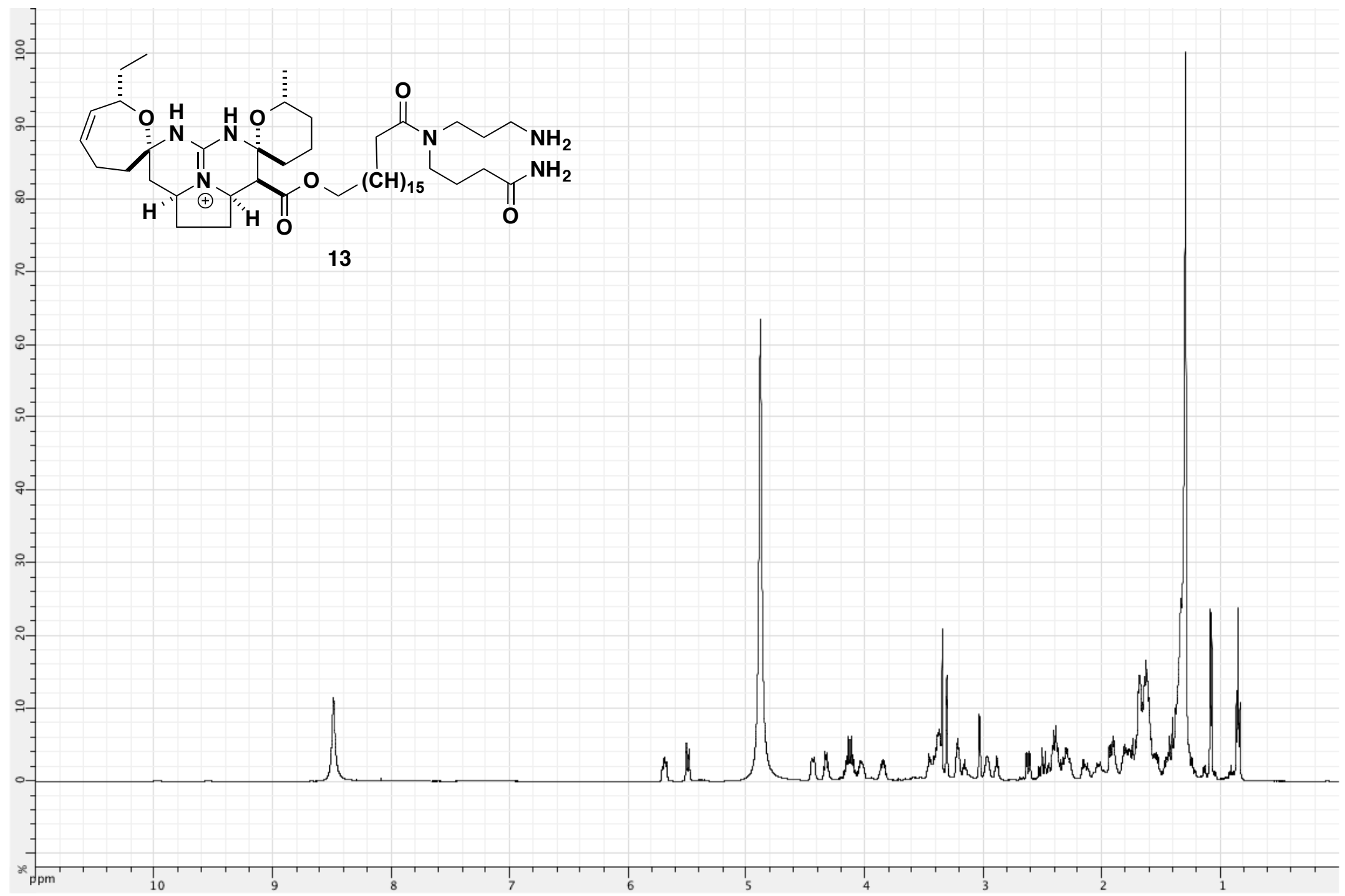

S60. ${ }^{13} \mathrm{C}$ NMR spectrum of crambescidin $826(\mathbf{1 3})$ in $\mathrm{CD}_{3} \mathrm{OD}(125 \mathrm{MHz})$. 


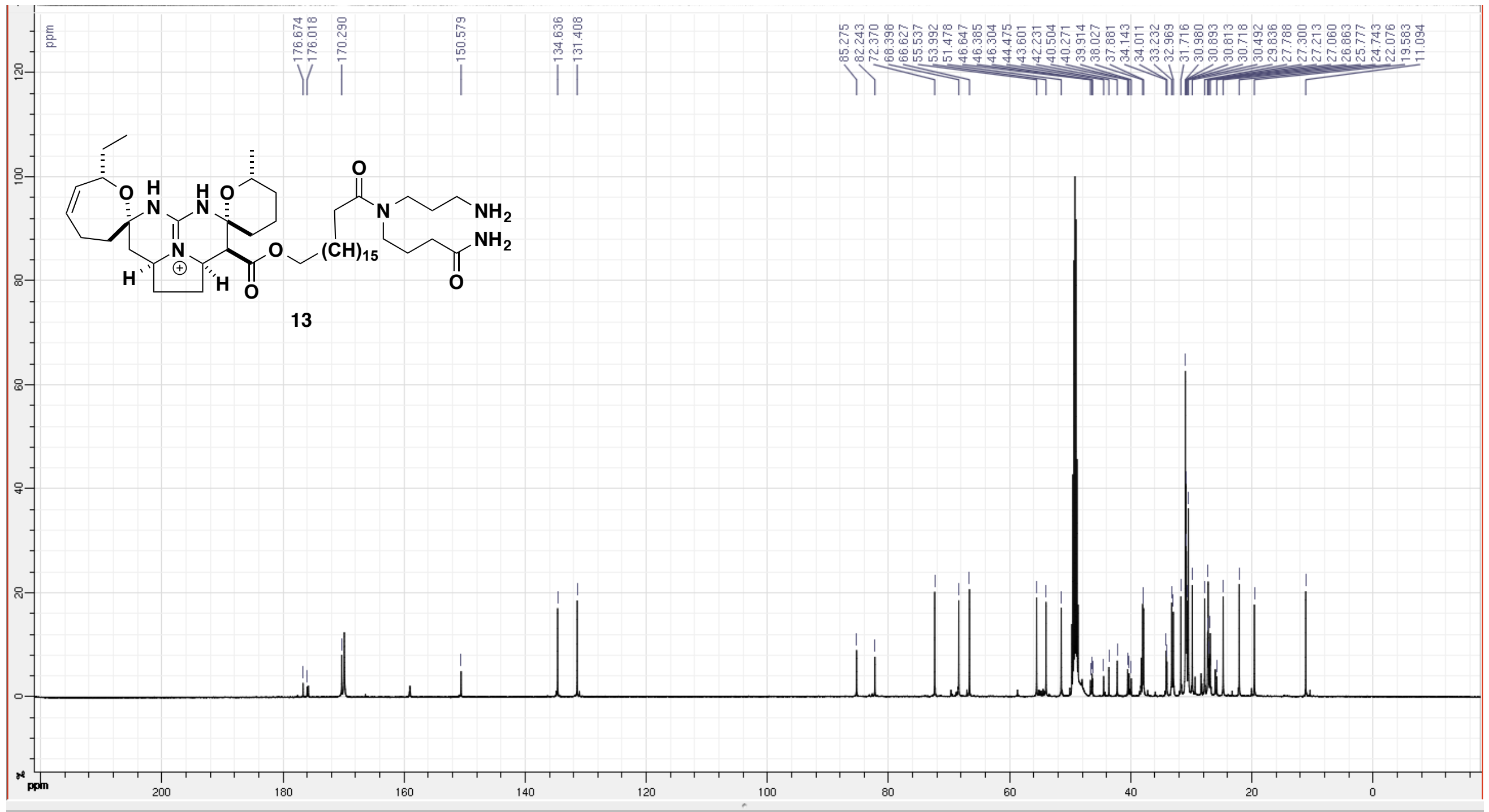

S61. ${ }^{1} \mathrm{H}$ NMR spectrum of crambescidic acid (14) in $\mathrm{CD}_{3} \mathrm{OD}(500 \mathrm{MHz})$. 


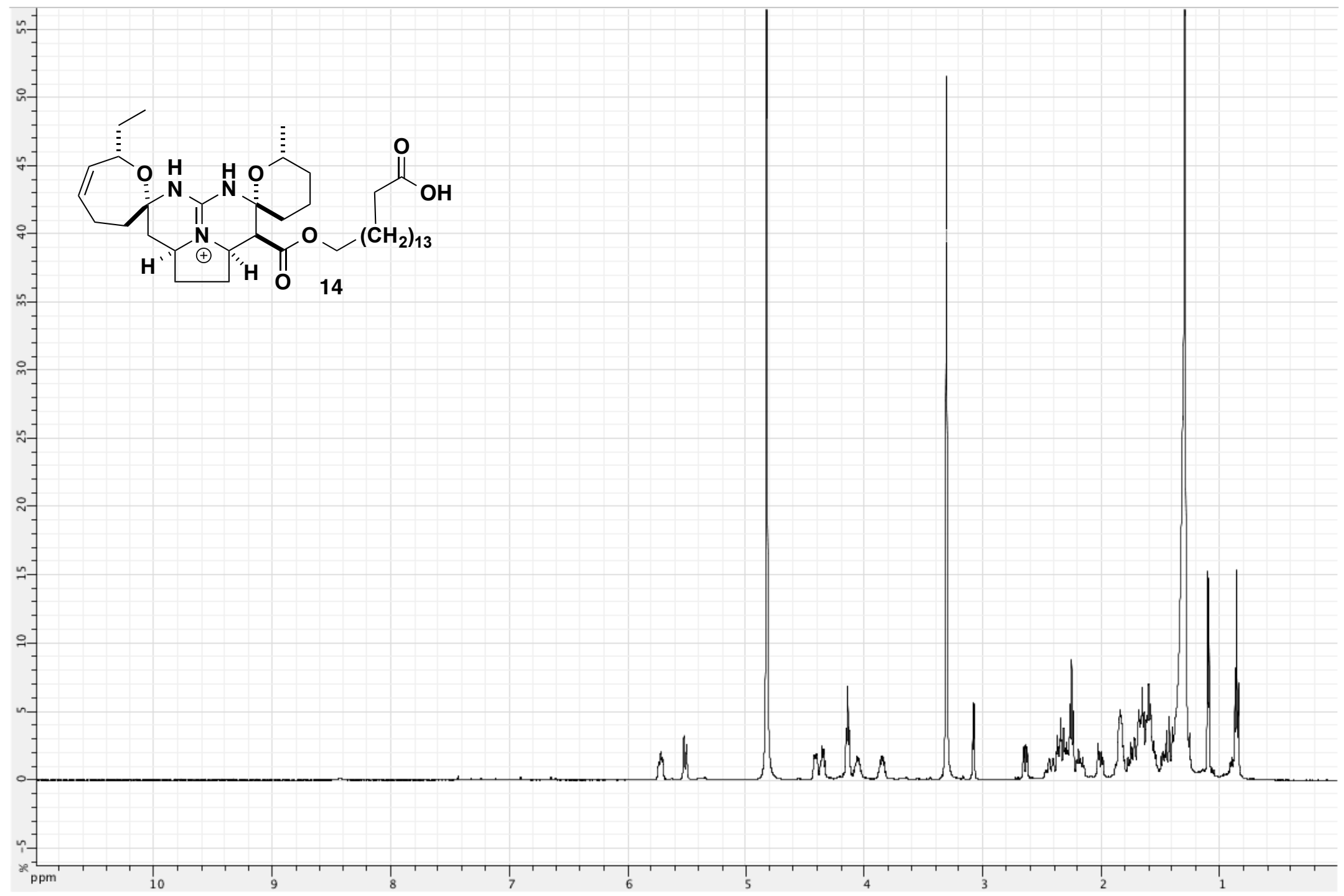

S62. ${ }^{13} \mathrm{C}$ NMR spectrum of crambescidic acid (14) in $\mathrm{CD}_{3} \mathrm{OD}(125 \mathrm{MHz})$. 


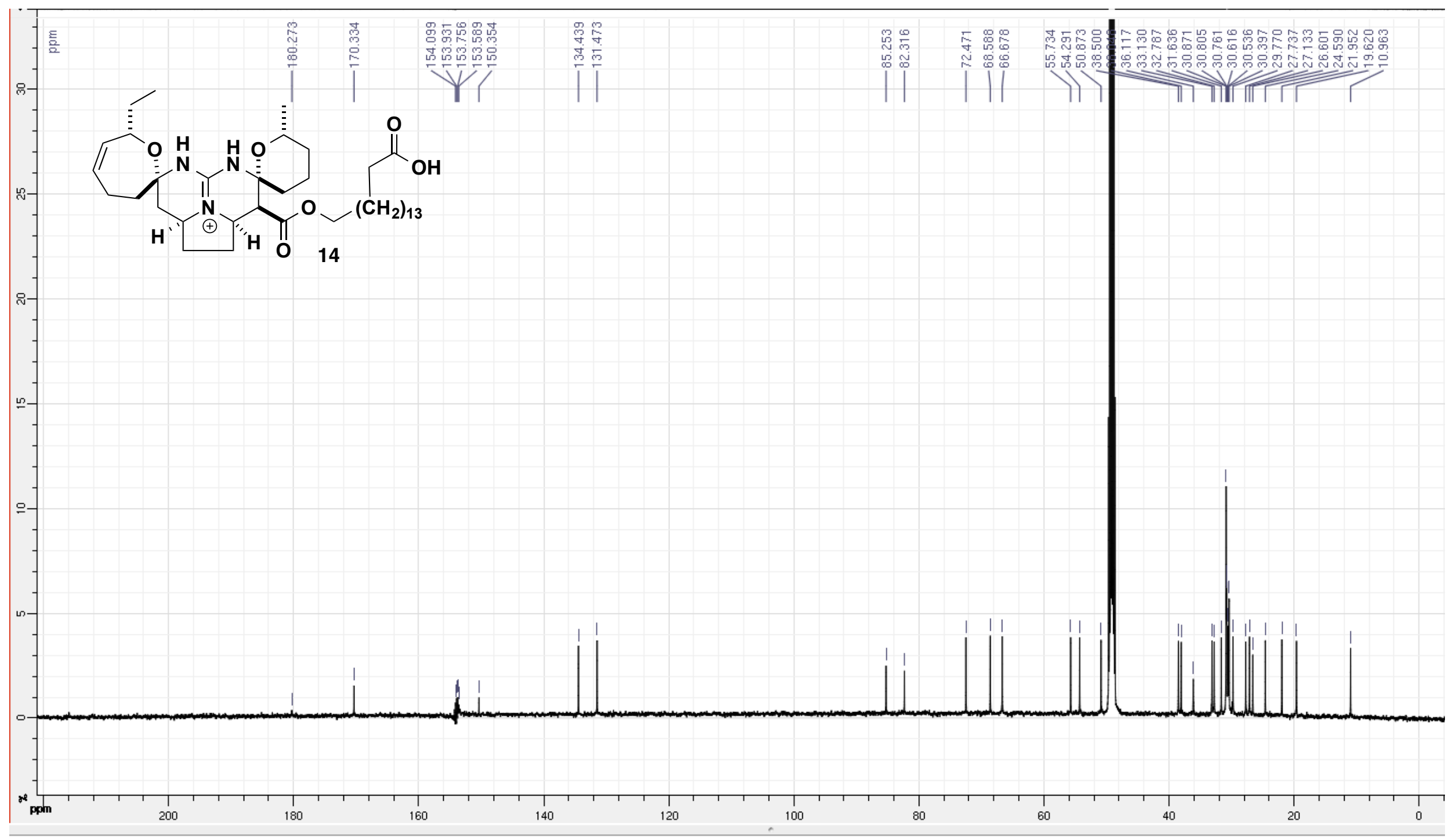

S63. ${ }^{1} \mathrm{H}$ NMR comparision for norcrambescidic acid (7) and crambescidic acid (14) in $\mathrm{CD}_{3} \mathrm{OD}(500 \mathrm{MHz})$. 


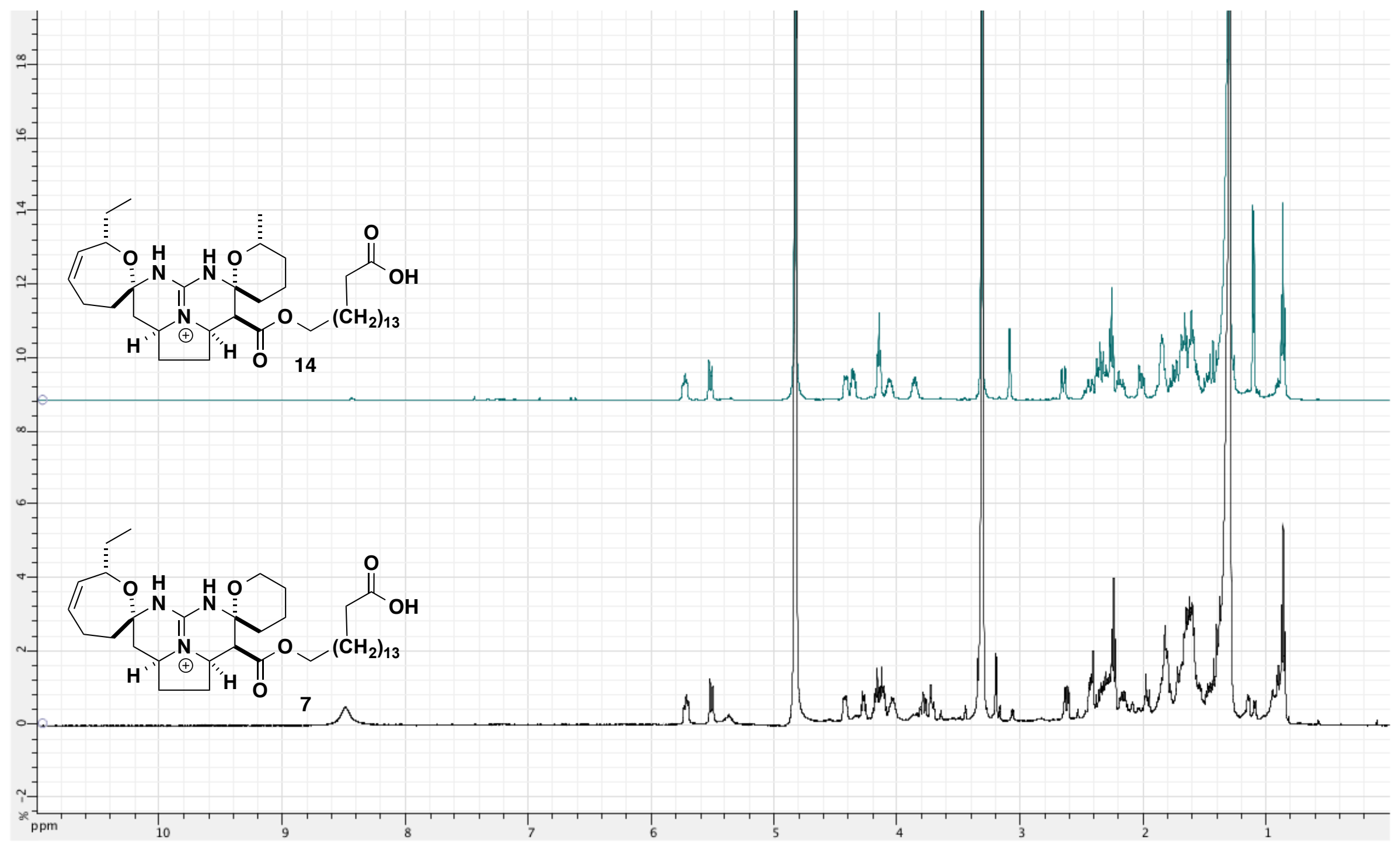

S64. ${ }^{1} \mathrm{H}$ NMR spectrum of crambescidin $359(\mathbf{1 5})$ in $\mathrm{CD}_{3} \mathrm{OD}(500 \mathrm{MHz})$. 


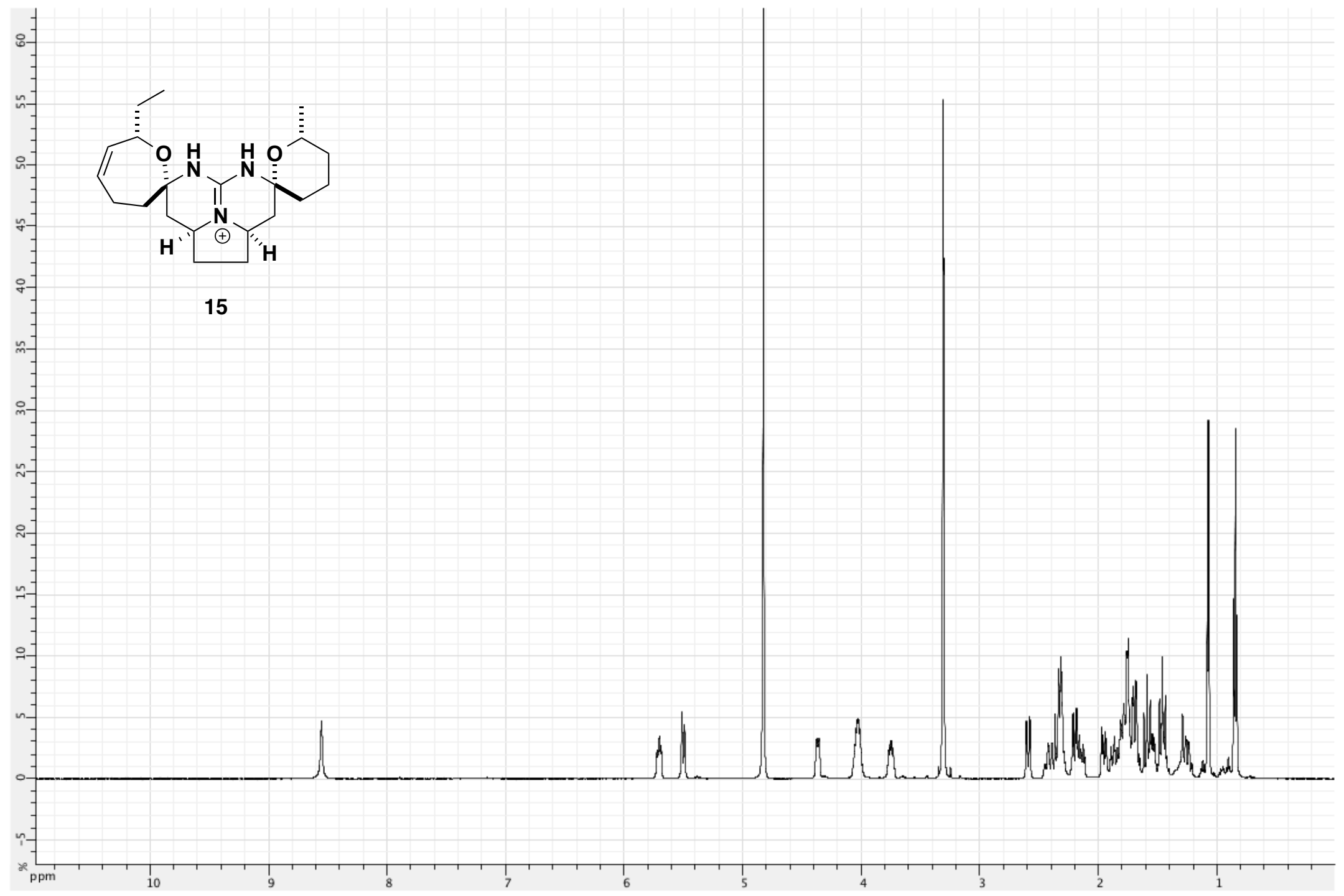

S65. ${ }^{13} \mathrm{C}$ NMR spectrum of crambescidin 359 (15) in $\mathrm{CD}_{3} \mathrm{OD}(125 \mathrm{MHz})$. 


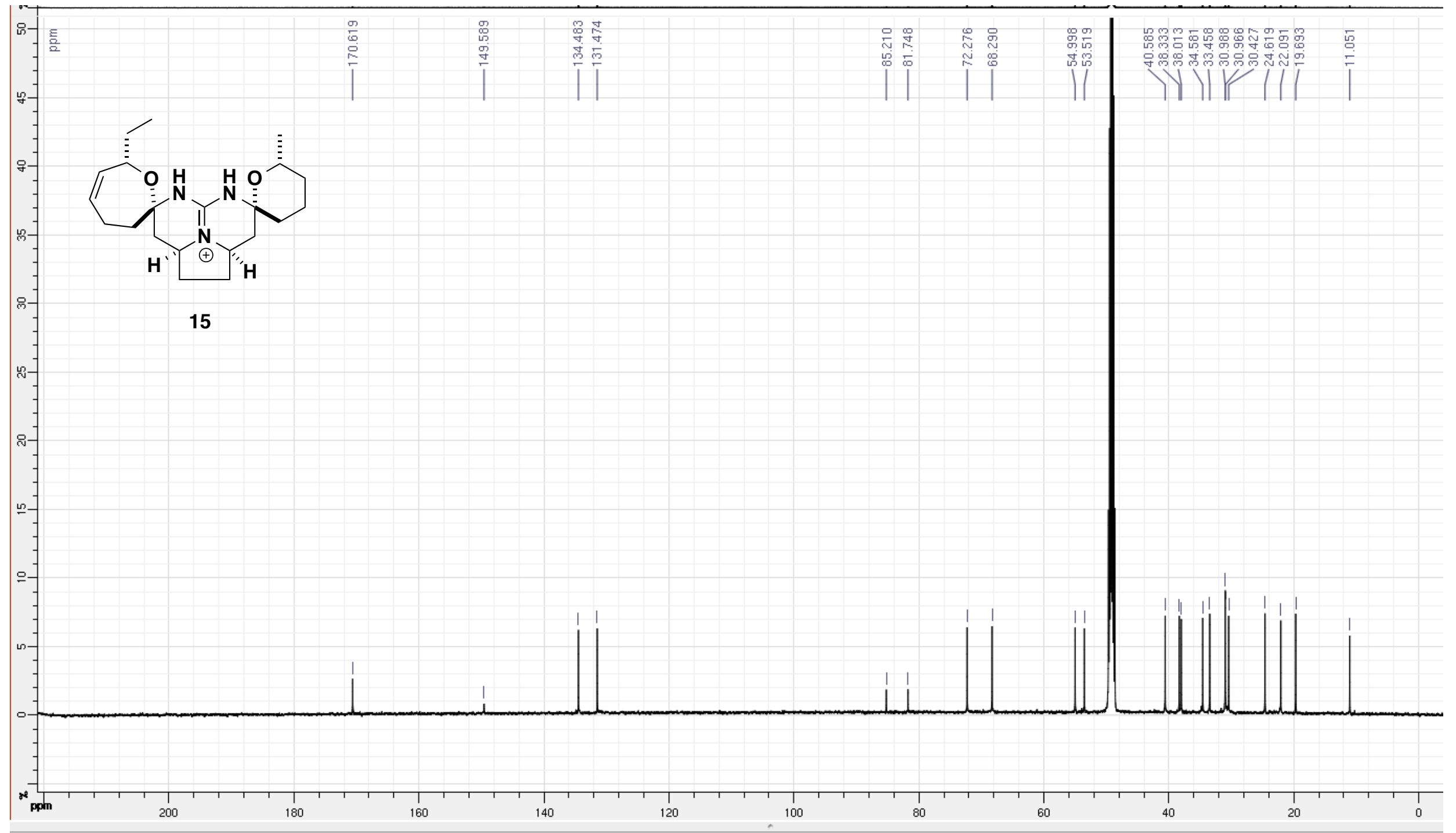

S66. ECD spectra for $(-)$-crambescidins $(\mathbf{6}, \mathbf{1 2}, \mathbf{1 4}, \mathbf{1 5})$, and the $(-)$-norcrambescidin $(7)$ 


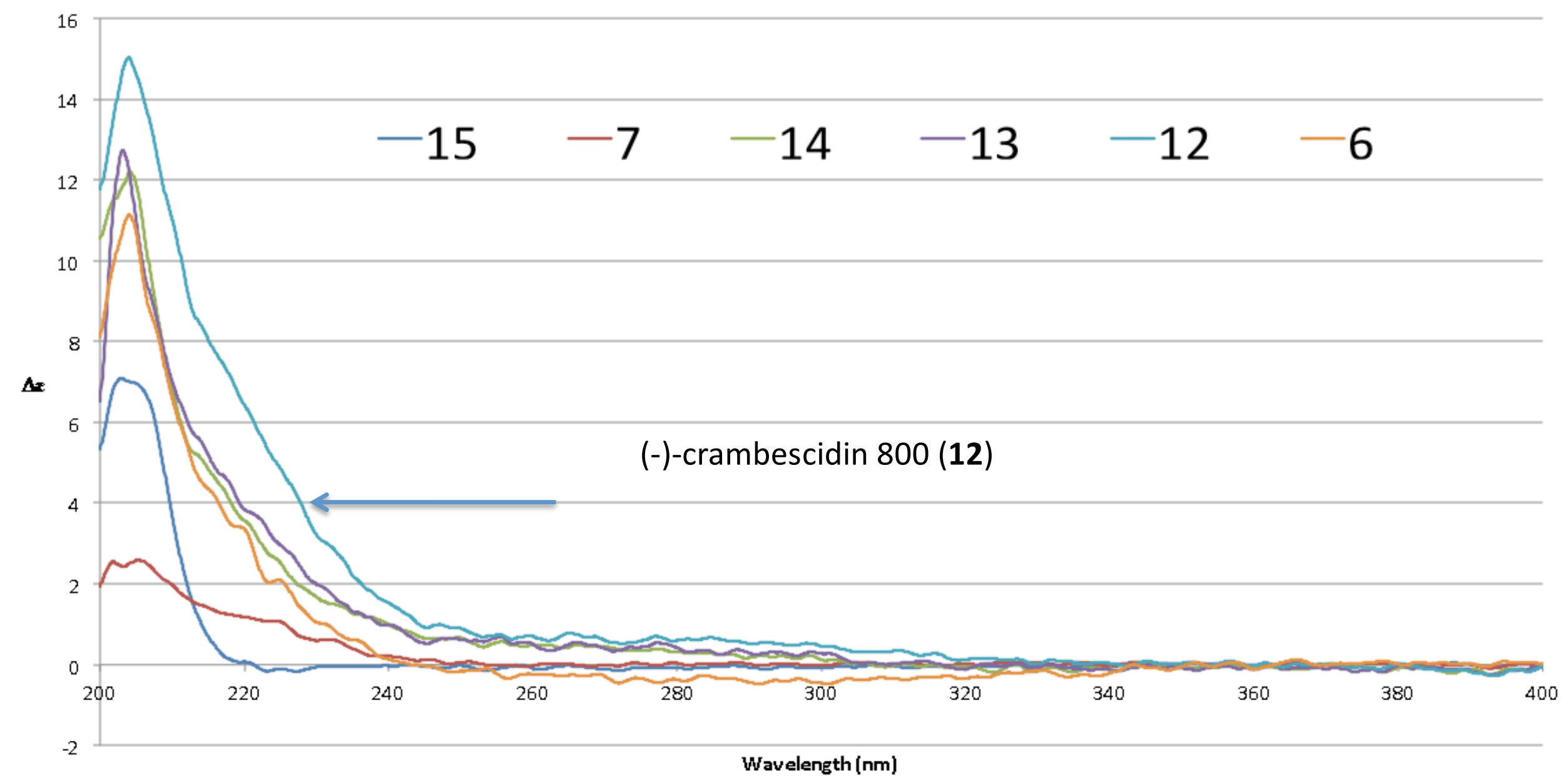

S67. Expanded ${ }^{1} \mathrm{H}$ NMR comparison of crambescidin 800 (12), crambescidin 814 (6) in $\mathrm{CD}_{3} \mathrm{OD}(500 \mathrm{MHz})$. 


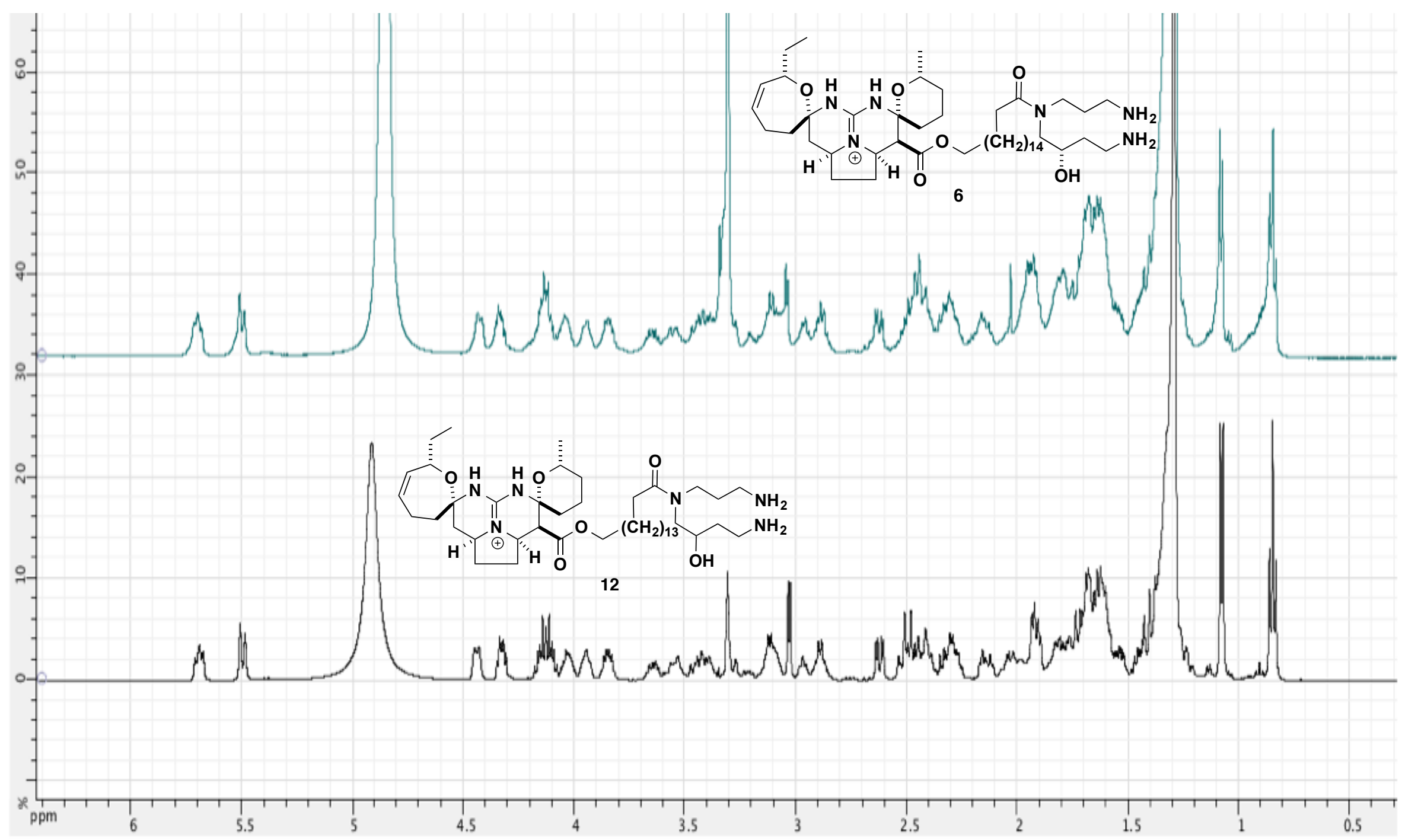

